Ng, A. M. B., M. Pontius, S. L. De Ruiter, and D. S. Proppe. 2020. Noise, avian abundance, and productivity at banding stations across the Continental United States. Avian Conservation and Ecology 15(2):4. https://doi.org/10.5751/ACE-01633-150204

Copyright (C) 2020 by the author(s). Published here under license by the Resilience Alliance.

Research Paper

\title{
Noise, avian abundance, and productivity at banding stations across the Continental United States
}

\author{
Amber M. B. $\mathrm{Ng}^{1,2}$, Michael L. Pontius ${ }^{1}$, Stacy L. De Ruiter ${ }^{1}$ and Darren S. Proppe ${ }^{1,3}$ \\ ${ }^{1}$ Calvin University, ${ }^{2}$ Western Michigan University, ${ }^{3}$ St. Edward's University
}

\begin{abstract}
Noise is an increasingly common component of the natural world, due in large part to human activity. Anthropogenic noise negatively impacts abundance, health, and reproduction in many songbird populations. A few studies have reported altered abundance at larger scales. But whether continental trends are being detected at banding stations, which also offer data on productivity and survivorship, is unknown. Further, it is not known whether localized trends correlate with population trends observed at larger scales. We used breeding season data from 1160 constant-effort banding stations (Monitoring Avian Productivity and Survivorship; MAPS) and a spatially explicit noise model to determine whether abundance and productivity were related to mean noise level or spatial heterogeneity (SD) in noise within a $1-\mathrm{km}$ station radius for 72 passerine species. We also determined whether particular life history traits were predictive of noise responses, and compared continental results to those from local studies. Increasing mean noise level was associated with declines in abundance for $27.1 \%$ of species and productivity in $22.1 \%$ species. Increasing heterogeneity was associated with declines in abundance for $14.3 \%$ species and productivity in $14.7 \%$ species. The relationship between noise and abundance was not correlated with the relationship between noise and productivity, and acoustic and life history traits were not related to noise responses. Continental results were similar to localized data in $43.1 \%$ and $21.4 \%$ of species for abundance and productivity, respectively. Although some patterns differed between the local and continental scale, our results indicate that the MAPS banding dataset is capable of detecting noise-associated impacts on abundance and productivity. This is currently the only large-scale dataset capable of quantifying the relationship between noise and productivity in the continental USA, although other datasets exist elsewhere that may also contribute to our understanding of noise impacts at the larger scale.
\end{abstract}

\section{Bruit, abondance et productivité des oiseaux aux stations de baguage des États-Unis continentaux}

RÉSUMÉ. Le bruit est une composante de plus en plus courante du monde naturel et causé en grande partie par l'activité humaine. Le bruit d'origine anthropique a un impact négatif sur l'abondance, la santé et la reproduction de nombreuses populations d'oiseaux chanteurs. Quelques études ont fait état d'une diminution d'abondance à plus grande échelle. On ignore si des tendances continentales sont détectées aux stations de baguage, lesquelles fournissent également des données sur la productivité et la survie. De plus, on ne sait pas si les tendances locales sont corrélées aux tendances de populations observées à plus grande échelle. Nous avons utilisé les données en saison de reproduction issues de 1160 stations de baguage à effort constant (programme « Monitoring Avian Productivity and Survivorship »; MAPS) et un modèle de bruit spatialement explicite pour déterminer si l'abondance et la productivité étaient liées au niveau de bruit moyen ou à l'hétérogénéité spatiale (SD) du bruit dans un rayon d'1 km de la station pour 72 espèces de passereaux. Nous avons également déterminé si des traits particuliers du cycle de vie étaient prédictifs des réactions au bruit, et nous avons comparé les résultats continentaux à ceux d'études locales. L'augmentation du niveau de bruit moyen a été associée à une diminution de l'abondance chez $27,1 \%$ des espèces et de la productivité chez $22,1 \%$ des espèces. Une hétérogénéité croissante a été associée à des baisses d'abondance chez 14,3\% des espèces et des baisses de productivité chez 14,7\% des espèces. La relation entre le bruit et l'abondance n'a pas été corrélée avec la relation entre le bruit et la productivité, et les caractéristiques acoustiques et du cycle de vie n'ont pas été liées aux réactions au bruit. Les résultats à l'échelle continentale étaient similaires aux données à l'échelle locale chez $43,1 \%$ et $21,4 \%$ des espèces pour l'abondance et la productivité, respectivement. Bien que certaines tendances diffèrent aux échelles locale et continentale, nos résultats indiquent que l'ensemble de données de baguage MAPS a la capacité de détecter les impacts du bruit sur l'abondance et la productivité. Il s'agit actuellement du seul ensemble de données à grande échelle permettant de quantifier la relation entre le bruit et la productivité aux États-Unis continentaux, bien qu'il existe d'autres jeux de données qui pourraient également contribuer à notre compréhension des impacts du bruit à plus grande échelle.

Key Words: anthropogenic; bird; demographic; MAPS; population; scale

\section{INTRODUCTION}

Human population growth and development have resulted in a rapid and widespread increase in noise levels (Buxton et al. 2017). In many animals, noise pollution alters acoustic communication, abundance, and migratory behaviors (Reijnen and Foppen 1995,
Slabbekoorn and Peet 2003, Shannon et al. 2016). Songbirds are especially sensitive to anthropogenic noise because their mating and territory defense rely on acoustic communication (Francis et al. 2011). In particular, occupancy and abundance often decline when noise levels increase (Bayne et al. 2008, McClure et al. 2013). 
Noise aversion may even outweigh the effects of land cover when songbirds are selecting places to breed (Kleist et al. 2017). A handful of studies also suggest that ambient noise impacts fitness through reduced pairing (Habib et al. 2007) and reproductive success (Halfwerk et al. 2011). Consequently, increasing anthropogenic noise may threaten songbird persistence (Barber et al. 2010). Throughout this paper we use the term "noise" to refer specifically to ambient sound levels at a location, whether environmental or anthropogenic in origin.

Most noise studies on abundance and productivity occur at small spatial extents $\left(<50 \mathrm{~km}^{2}\right.$; e.g., Francis et al. 2009, McClure et al. 2013, Proppe et al. 2013). Intensive local studies provide fine-scale detail that can account for potentially confounding localized processes such as interspecific competition (Schoener 1983), legacy effects (Cuddington 2011), density-dependent processes (Haldane 1956) and source-sink dynamics (Pulliam 1988). However, these processes often differ geographically, which limits the generalizability of results gained from local studies. For example, negative impacts of noise on reproductive success have been documented in Great Tits (Parus major; Halfwerk et al. 2011) and House Sparrows (Passer domesticus; Schroeder et al. 2012), but recent studies did not detect negative impacts on productivity in either species (Meillère et al. 2015, Halfwerk et al. 2016).

Increasing the spatial extent of a study often comes at a cost to fine-scale detail, but access to larger datasets can reveal trends not immediately visible at smaller scales (Rahbek 2005). For example, juvenile and adult male American Redstart (Setophaga ruticilla) distributions are negatively correlated at small scales because of territoriality, but positively correlated at larger scales because of similar habitat requirements (Sherry and Holmes 1988). Four studies have examined the effect of noise on songbird abundance and occurrence at the continental scale, although all were using large-scale datasets to determine whether vocal pitch or particular life history traits were related to abundance and presence in noisy and urbanized areas ( $\mathrm{Hu}$ and Cardoso 2009, Cardoso 2014, Francis 2015, Moiron et al. 2015). These studies relied on many sources of localized abundance data or published reports describing urban tolerance. Although all four studies documented declines in abundance or occurrence of some species near noise sources and in cities, the importance of vocal pitch differed between studies (discussed in Cardoso et al. 2018). Clearly, a combination of data sources, from both localized and large-scales studies, is needed to best understand the impact of anthropogenic noise on songbird persistence.

The Institute of Bird Populations has been developing a largescale dataset that could provide additional information on noise impacts at the continental level. The MAPS (Monitoring Avian Productivity and Survivorship) program has been tracking avian demographics, including abundance, productivity, and survivorship during the breeding season at banding stations since 1989. This ongoing program has expanded to over 1200 stations, more than 2 million bird captures, and over 230 publications on avian demographics and vital rates. Effort is standardized across all MAPS stations so that data can be compared across time and space. To our knowledge, this is the only large-scale dataset that also provides information on productivity in North America, although similar datasets exist elsewhere (e.g., Eglington et al. 2015). Quantifying productivity provides a measure of birth rates in a population, and is often strongly tied to population trends (Sallabanks et al. 2000).

Two additional datasets provide information about noise and urbanization at each MAPS banding station. First, the United States National Park Service (NPS) has developed a geospatial sound model that uses 109 natural and anthropogenic factors associated with ambient noise levels (Mennitt et al. 2014) to predict the $\mathrm{L}_{50} \mathrm{dBA}$ at a $270 \mathrm{~m}$ resolution for the a typical summer day from 2003 to 2013 across the entire continental United States (CONUS). Second, the United States Geological Survey (USGS) produced CONUS land cover maps in 2001, 2006, and 2011 that include habitat classifications and a measure of development at $30 \mathrm{~m}$ resolution (Homer et al. 2015).

We assessed the relationship between model-predicted noise levels and the abundance and productivity of 72 passerine species by computing the weighted mean of noise within $1 \mathrm{~km}$ of each banding station (Fig. 1). Visual investigation of noise data revealed that a number of MAPS stations had high levels of noise heterogeneity within the $1 \mathrm{~km}$ radius. To determine whether this noise pattern impacted species differently than uniformly high or low noise levels, we assessed the impact of noise heterogeneity on abundance and productivity. Species were selected because they were among the 30 most commonly captured at MAPS stations or because localized data on noise impacts were available. Local studies included 49 species, seven which were also among the 30 most common species. After controlling for the effects of location, time, development, and habitat, we determined whether noise was an important predictor of abundance or productivity for each species. We also calculated whether the directional associations of noise with abundance and productivity were correlated, such that knowing the relationship of noise with one measure facilitated a predictive understanding of the other. Finally, we determined whether the relationship with noise was predicted by particular life history traits, and we compared continental data to local data to determine the level of agreement between these two scales.

Fig. 1. Map of noise predictions for the continental United States produced by the United States National Park Service overlaid with the location of each Monitoring Avian Productivity and Survivorship (MAPS) banding station (yellow circles) located within the region. The expanded view of two MAPS stations are provided as examples of the two noise variables used for the analysis: mean noise level and the heterogeneity of noise.

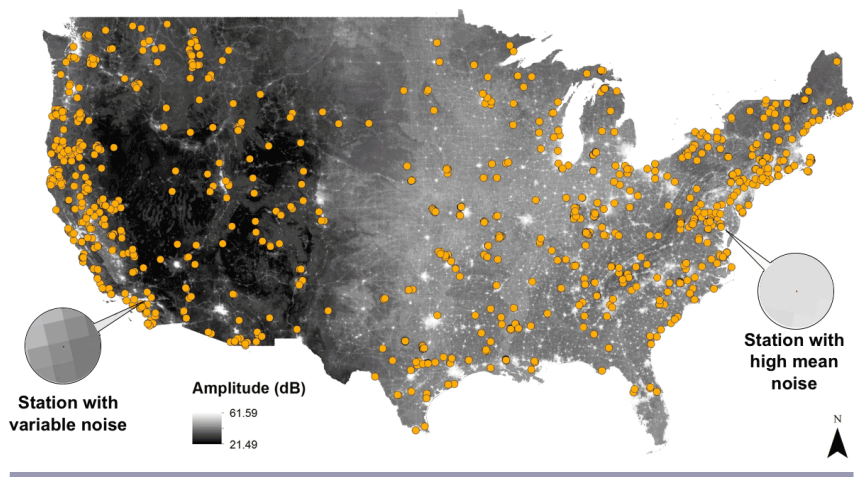




\section{METHODS}

\section{Datasets}

Data on avian demographics from 1989 to 2016 was provided by The Institute of Bird Populations MAPS banding program. Each participating station consists of ten 12-m mist nets located on an area approximately 8 ha in size that is contained within a contiguous landscape of $>20$ ha. Mist nets are opened for six hours (beginning at dawn) on one day in a 10-day period. Based on the latitude of the station, six to ten 10-day periods are run consecutively during the breeding season (May-August) each year. Age, sex, and reproductive status are recorded for each bird, and a metal USGS band is attached to the tarsus before release to facilitate the collection of recapture data.

The United States National Park Service used 109 natural and anthropogenic factors associated with ambient noise levels (Mennitt et al. 2014) to predict the $\mathrm{L}_{50} \mathrm{dBA}$ at a $270 \mathrm{~m}$ resolution for the entire continental United States. Noise predictions were tested at 319 acoustic monitoring sites within 216 national parks and 34 urban locations in 28 unique cities spanning the continental United States. The model explained $71.74 \%$ of the noise variance across all sites. Anthropogenic sources increased the noise level predicted by biophysical processes alone in $78 \%$ of the landmass. The model is limited to a single noise measure per pixel, calculated for a typical summer day from 2003 to 2013.

The USGS periodically produces a $30-\mathrm{m}$ resolution layer of habitat classifications and development levels as part of the National Land Cover Database (NLCD). We used 2001 data because this was the year closest to the median year of bird captures available in the MAPS dataset. For our analysis we incorporated two measures of development and seven measures of habitat to control for their effect on bird demographics. Development variables in the NLCD were derived from the percent cover of impervious surfaces, which is a strong predictor of the impact urbanization has on songbirds (Melles et al. 2003, McClure et al. 2015), and the type and quantity of human structures (Homer et al. 2012). We combined open $(<20 \%$ anthropogenic cover) and low (20-49\%) development into a single category and $\operatorname{mid}(50-79 \%)$ and high ( $80-100 \%)$ development into a second category. Habitat variables included the proportion of land covered by water, wetlands, forest, herbaceous, shrubland, barren, and planted/cultivated.

To determine whether life history traits were predictive of noiseassociated changes in abundance and productivity, we categorized bird species into several guilds based on (1) song frequency, (2) breeding habitat, (3) diet, (4) nest location, and (5) migratory behavior. Vocal frequencies were derived from two to eight highquality songs for each species housed on Thayer Birding Software (Birds of North America, Gold Edition). Because multiple elements of song frequency have been implicated in noise response and can be modulated independently, we measured peak frequency, bandwidth $(25 \%-75 \%$ summed energy), lowest frequency ( $5 \%$ of summed energy), lowest quartile $(25 \%$ of summed energy), and highest frequency ( $95 \%$ of summed energy) in Raven Pro (V1.4, Ithaca, NY). For species lacking a song, the most common call was utilized. All other life history traits were collected from the Cornell Lab of Ornithology's website (https:// www.allaboutbirds.org/news/). Breeding habitat classifications included: grassland, forest, open woodland, scrub, and town. Birds were classified as insectivores or noninsectivores based on the primary components of their diet. Nest location was classified as cavity, ground, shrub, or tree. For migration, species were categorized as nonmigrants, migratory, or partial migrants. Species were categorized as partial migrants when range maps indicated that populations were resident in $25-50 \%$ of their range, but otherwise migratory.

To eliminate the potential for implicit bias, we evaluated regional studies for inclusion in our study during September of 2016, prior to initiating the processing of banding data. We established two a priori criteria for inclusion. First, each study must have occurred within the North American region where both MAPS and noise data were available, or examined species commonly found within this region. Second, each study must have included directional results for abundance or productivity in relation to noise or proximity to noise-producing sources, e.g., roads or oil wells. Although the majority of the literature on anthropogenic noise addresses behavioral responses in songbirds, we identified 13 suitable studies using multiple subsets and variants of the search terms; songbird, noise, abundance, reproduction, and productivity in Web of Science and Google Scholar (Appendix 1). Many of these studies examined multiple species, resulting in local demographic information for 49 species. Abundance data was available for 44 species, and productivity was available for 16 species. Of these species, 11 had data on both abundance and productivity.

\section{Data processing}

For each species, abundance was calculated as the number of adults captured per 600 net hours, and productivity was the ratio of juveniles (AGE code 4 or 2) to adults (all other AGE codes, except 0 and 9 where age is unknown; DeSante and Kaschube 2009). Although effort was standardized, we cannot assume that capture rates were equal across all sites. However, there are no indications that noise alters capture rates in a systematic fashion when birds are on the breeding grounds (although it may impact site selection during migration, when birds are actively selecting stopover sites; McClure et al. 2017). This information, along with our large sample size, should overcome any potential spurious associations based on differences in site-based capture rates. Survivorship was not analyzed because birds are often recaptured long after they were initially banded, and at sites with noise levels and surface characteristics that are quite different from the original banding location. Thus, a myriad of factors beyond the particular characteristics at the site of origin are likely to impact survivorship. For any given species, only stations within that species' range (breeding status code $\mathrm{B}, \mathrm{U}, \mathrm{O}$, and $\mathrm{T}$ ) were included (DeSante et al. 2015). A minority of MAPS stations are operated more than once per 10-day period. For such stations we only included captures during the first sampling subperiod (code A) to standardize effort across all stations. Because MAPS regulations suggest that data from locations $<1 \mathrm{~km}$ from the banding station's center are not independent (DeSante et al. 2015) and breeding birds are unlikely to venture $>1 \mathrm{~km}$ from their breeding territory, we calculated the mean noise level within a 1 $\mathrm{km}$ radius of each MAPS station. The mean was linearly weighted to increase the influence of more central pixels by dividing the noise level in each pixel by the distance between that pixel and the 
station center, and then averaging the results for all pixels. To evaluate spatial heterogeneity we calculated the standard deviation of noise levels in all pixels contained within the $1 \mathrm{~km}$ radius. Although heterogeneity can be measured in many ways, we chose standard deviation because it is used regularly in the literature (e.g., Yang et al. 2015, Khan et al. 2019), is easy to interpret, and effectively creates a standard measure of variability comparable across sites with high and low mean noise levels.

Habitat and development variables were calculated as the proportion of all pixels within a $1 \mathrm{~km}$ radius surrounding each station. We used a principal component analysis on the development and habitat variables to obtain a set of uncorrelated variables for inclusion as predictors in regression models. We retained eight principal components that accounted for $>80 \%$ of the variation (Appendix 2). Each component was given a relevant name based on the top one to three contributing variables. In the order of explanatory power, these components increased with "development" (PC1; 20.08\% variance explained), "wetland" (PC2; 11.75\%), "shrub" (PC3; 10.78\%), "barren" (PC4; 9.22\%), "marsh" (PC5; 8.15\%), "grassland" (PC6; 7.49\%), "water" (PC7; $7.46 \%$ ), and "urban" (PC8; 6.84\%).

\section{Statistical analysis}

For each of 72 species of interest, we fitted two generalized additive mixed-effects models (GAMMs): one with abundance as the response variable, and one with productivity. Abundance models were Poisson or quasi-Poisson (quasi-Poisson, in cases where the overdispersion parameter estimate exceeded 2) GAMMs with log link functions (Hastie and Tibshirani 1990, Zuur et al. 2009, Wood 2017). Predictors included the weighted mean noise level, the standard deviation of the noise level, the eight habitat and development principal components, and location (2-dimensional smooth term; thin-plate regression spline with basis of dimension 16; Wood 2017). An offset (logarithm of net-hours) accounted for differential survey effort between stations and years (Zuur et al. 2009). Productivity models were Poisson or quasi-Poisson GAMMs with log link functions (Zuur et al. 2009). The response variable in these models was the count of juvenile birds, with an offset (logarithm of the count of adult birds), so we effectively modeled the number of juveniles per adult (Zuur et al. 2009). Candidate predictors were the same as for abundance models. Year (smooth term; thin-plate regression spline with basis of dimension 5) was included as an additional predictor in all models to account for nonmonotonic patterns over time in a variety of factors, including land use changes. All models also included a random effect of station. Although these GAMM models detected counts, rather than yielding absolute bird abundance or productivity estimates, species-specific modeling and inclusion of covariates related to location, habitat, and time should still allow reliable assessment of noise effects on indices of abundance and productivity (Dunn and Ralph 2004). We used variance inflation factors to confirm the absence of problematic multicollinearity between predictors (particularly noise and development/habitat PCs; Dormann et al. 2013). Four candidate models were considered for abundance and productivity of each species, including (a) both noise variables, (b) mean noise only, (c) heterogeneity of noise only, and (d) no noise variables. Habitat, development, and spatial predictors were retained in all candidate models to control for their effects on abundance or productivity. Best models for each species and response variable were selected using AIC (or QIC, for quasiPoisson models). Where best models included a noise term, but the AIC/QIC improvement relative to the next-best model without the noise term was below $2(\triangle \mathrm{AIC} / \mathrm{QIC}<2)$, it was noted by labeling the effect as "weak"; (Appendix 3; Burnham and Anderson 2002, Burnham and Anderson 2004). Model fitting was carried out in $\mathrm{R}$ version 3.6.1 (R Core Team 2019) using package mgcv (Wood 2011, 2017).

To investigate the relationship between noise-associated changes in abundance and productivity, we plotted and modeled the productivity regression coefficients as a function of the abundance coefficients. Separate models were fitted for weighted mean noise levels and noise heterogeneity coefficients. Although top abundance and productivity models excluded noise terms for some species, those terms were included here to produce effect sizes for all species. To account for uncertainty in the GAMM coefficient estimates used as input to these models relating abundance and productivity results, we used measurement error models, using the standard error of the estimates as the measurement error standard deviations (McElreath 2020). Models were fitted in $\mathrm{R}$ using package brms (Bürkner 2018). To assess the relationship between the abundance and productivity noise-effect coefficients, we examined $95 \%$ credible intervals for the abundance coefficients in the measurement error models, and additionally compared each model to its intercept-only version via leave-one-out cross-validation (brms function loo(); Vehtari et al. 2017).

To investigate relationships between noise responses and life history traits, we fitted a measurement error model (using standard errors in the GAMM coefficient estimates as the measurement errors, as above) for each noise coefficient (abundance and productivity, weighted mean noise and noise standard deviation) as a function of the suite of vocal and life history traits described above. These included peak frequency, bandwidth, lowest frequency, lowest quartile, highest frequency, habitat, insectivory, nesting, and migration. Models were fitted with horseshoe shrinkage priors (Piironen and Vehtari 2017) in $\mathrm{R}$ package brms (Bürkner 2018), and the best model for each noise coefficient was selected after examining model output (estimates and credible intervals), considering output from projection predictive forward variable selection (using $\mathrm{R}$ package projpred; Piironen et al. 2019), and as verification, leave-one-out cross-validation scores for the top models identified by the variable selection.

To determine whether regional noise impacts were similar to our landscape results, we compared the directional effect $(+, 0,-)$ of increased noise on abundance and productivity across scales and noted the percentage of cases demonstrating agreement, and vice versa.

\section{RESULTS}

\section{Continental models for abundance and productivity}

The distribution of model-predicted noise levels at MAPS stations $($ mean $=37.7, \mathrm{SD}=5.1$, range $=23.9-54.8, n=47,048)$ was similar to that of the full NPS dataset (mean $=35.6, \mathrm{SD}=$ 
5.3, range $=20.1-67.0, n=183,300,975)$. Our best species-specific landscape models retained a negative relationship between the weighted mean of noise and abundance in 19 species $(27.1 \%)$, a positive relationship in 19 species $(27.1 \%)$, and no clear statistical relationship in 32 species $(45.8 \%$; Table 1 , species-specific effect sizes and model rankings can be found in Appendix 3 and 4, respectively). As spatial heterogeneity in noise increased, abundance decreased in 10 species $(14.3 \%)$, increased in 27 species $(38.6 \%)$, and had no clear statistical relationship in 33 species $(47.1 \%)$. MAPS data was insufficient to build continental-scale abundance models for two species (Golden-cheeked Warbler [Setophaga chrysoparia] and Tennessee Warbler [Leiothlypis peregrina]) and these species were not used in the calculation of percentages above $(n=70)$. Productivity was negatively related to increasing mean noise levels in 15 species $(22.1 \%)$, positively related in 15 species $(22.1 \%)$, and not statistically related in 38 species $(55.8 \%)$. Productivity and noise heterogeneity were negatively related in 10 species $(14.7 \%)$, positively related in 22 species $(32.4 \%)$, and not statistically related in 36 species $(52.9 \%)$. MAPS data was insufficient to build continental-scale productivity models for four species (Golden-cheeked Warbler, Tennessee Warbler, Eastern Meadowlark [Sturnella magna], and White-crowned Sparrow [Zonotrichia leucophrys]) and these species were not used in the calculation of percentages above ( $n$ $=68$ ). Model failures in these species resulted from low capture numbers, which was primarily due to a narrow geographic range, breeding primarily outside the continental United States, or the inability to capture juvenile grassland species with MAPS protocols.

In 16 species, no changes in abundance and productivity attributable to the weighted mean of noise were identified. Noise heterogeneity had no detectable association in 19 species. Measurement error models indicated no correlation between coefficients for effects of weighted mean noise on abundance and productivity (estimated slope coefficient (with shrinkage) was 0.00 , and the intercept-only model was the best model, with a leave-one-out information criteria (LOOIC) difference of just 0.02; Figure 2). The corresponding results for the effects of noise spatial heterogeneity were similar (estimated slope coefficient 0.00 with shrinkage; full model LOOIC was lower than the interceptonly model, but only by 0.03 ; Figure 3 ). Neither acoustic nor life history traits improved on the intercept-only model for mean or heterogeneity of noise in abundance or productivity (estimated slope coefficient 0.00 with shrinkage; LOOIC intercept-only model lowest or within 0.13 of all other models; Appendix 5).

Principal components related to habitat and development were retained in all candidate models to control for the expected effects of these conditions rather than undertaking an explicit assessment of their contribution. However, 44 of 70 abundance models contained significant principal component predictors $(p<0.05)$ and 25 of 68 productivity models also contained principal component predictors (Table 1). In contrast, one or more noise terms were retained in 58 abundance models and 38 productivity models. Although beyond the scope of this report, information about the relative impact of habitat and development on particular species can be gleaned from these results (Appendix 3).
Fig. 2. Model coefficients for the effects of weighted mean noise level on the abundance and productivity of individual species ( $\triangle$ Abundance or Productivity/Decibel[dBA SPL]). Coefficients, and their associated standard errors, were derived from speciesspecific models that predicted the relationship between mean noise level and the demographic trait. Error bars indicate 95\% confidence intervals. Inset provides an expanded view of species near the origin.

\section{Weighted Mean Noise Level}

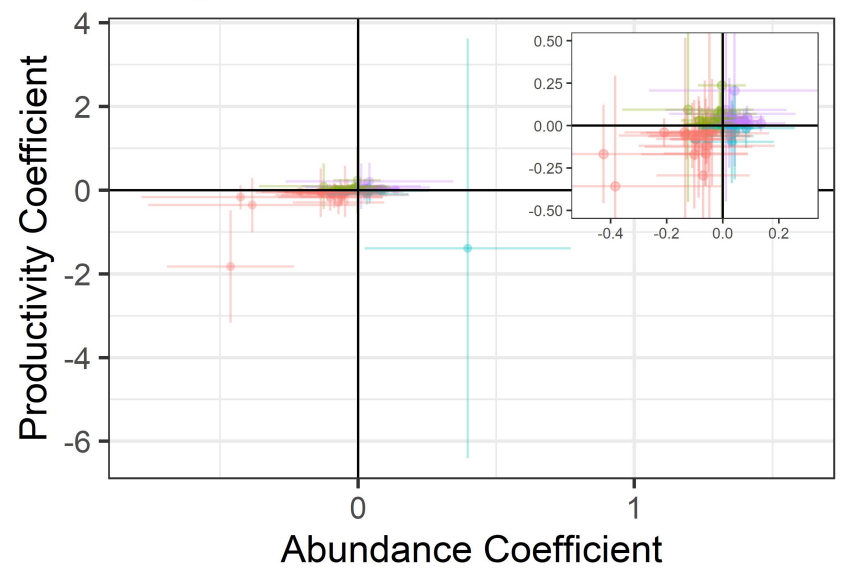

Fig. 3. Model coefficients for the effects of noise standard deviation (SD) on the abundance and productivity of individual species ( $\triangle$ Abundance or Productivity/SD Unit). Coefficients, and their associated standard errors, were derived from species-specific models that predicted the relationship between noise heterogeneity and the demographic trait. Error bars indicate $95 \%$ confidence intervals. Inset provides an expanded view of species near the origin.

\section{Noise Standard Deviation}

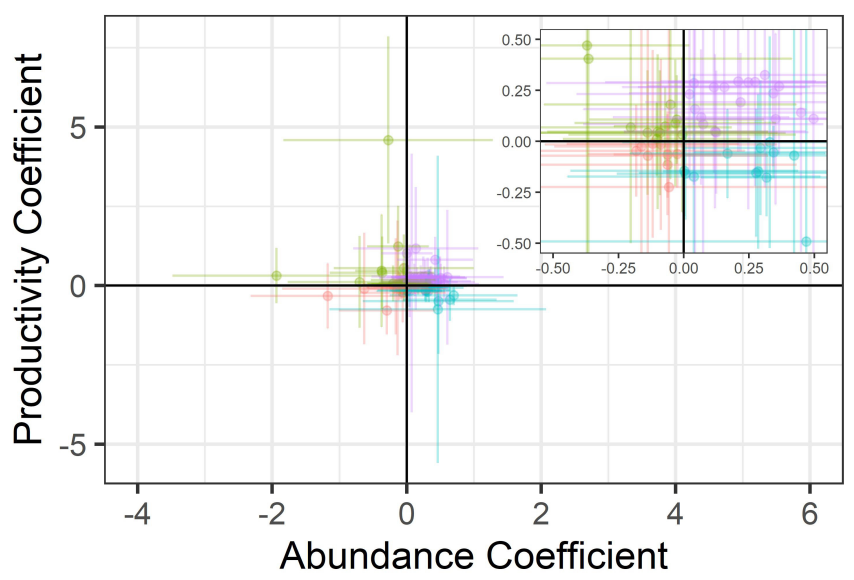

Quadrant $-+-\leftarrow-+-++-++$

Erratum: Insets were added to Figures 2 and 3 (PDF version) to improve comprehensibility and replaced those in the original publication on 4 August 2020. 
Table 1. Results from top models depicting the impact of noise level (mean) and heterogeneity (sd) on abundance and productivity for each species. Where available, local results and agreement with Monitoring Avian Productivity and Survivorship (MAPS) continental scale results are presented. For ease of reading, effects are presented as positive $(+)$, negative (-), or no effect $(0)$. Principal components significant at $p<0.05$ are included as positive (no sign) or negative (-) effects. Numerical effect sizes can be found in Appendix 4. See Appendix 3 for scientific species names. Where data from multiple local studies is presented, numbered superscripts refer to study superscripts found in Appendix 1.

\begin{tabular}{|c|c|c|c|c|c|c|c|c|c|c|}
\hline \multirow[b]{2}{*}{ Species } & \multicolumn{5}{|c|}{ Abundance } & \multicolumn{5}{|c|}{ Productivity } \\
\hline & $\begin{array}{l}\text { MAPS } \\
(\text { mean })\end{array}$ & $\begin{array}{l}\text { MAPS } \\
(s d)\end{array}$ & $\begin{array}{l}\text { Local } \\
\text { (mean) }\end{array}$ & $\begin{array}{c}\text { Agree: } \\
\text { MAPS/ } \\
\text { local }\end{array}$ & $\begin{array}{l}\text { Principle } \\
\text { components }\end{array}$ & $\begin{array}{l}\text { MAPS } \\
\text { (mean) }\end{array}$ & $\begin{array}{l}\text { MAPS } \\
(s d)\end{array}$ & $\begin{array}{l}\text { Local } \\
\text { (mean) }\end{array}$ & $\begin{array}{c}\text { Agree: } \\
\text { MAPS/ } \\
\text { local }\end{array}$ & $\begin{array}{l}\text { Principle } \\
\text { components }\end{array}$ \\
\hline Acadian Flycatcher & 0 & 0 & 0 & Yes & marsh & 0 & - & & & wetland, -urban \\
\hline American Goldfinch & + & - & & & wetland, -barren, -marsh & 0 & 0 & & & \\
\hline American Redstart & - & + & & & -shrub, -water & - & 0 & & & \\
\hline American Robin & + & 0 & - & Opposite & development, shrub & + & 0 & & & wetland \\
\hline Ash-throated Flycatcher & 0 & - & & & shrub, -barren, -marsh & 0 & 0 & - & No & \\
\hline Audubon's Warbler & 0 & + & & & -development & 0 & 0 & & & \\
\hline Bewick’s Wren & + & 0 & & & $\begin{array}{c}\text { development, wetland, shrub, } \\
\text { urban }\end{array}$ & 0 & 0 & & & \\
\hline Black-headed Grosbeak & 0 & + & - & No & wetland, - barren & + & + & - & Opposite & \\
\hline Black-capped Chickadee & + & 0 & 0 & No & development & 0 & - & & & \\
\hline Black-chinned & + & 0 & + & Yes & development & 0 & 0 & + & No & shrub \\
\hline \multicolumn{11}{|l|}{ Hummingbird } \\
\hline Bobolink & + & 0 & - & Opposite & $\begin{array}{l}\text { wetland, - shrub, - barren, } \\
\text { marsh, grassland, -water }\end{array}$ & 0 & 0 & - & No & \\
\hline Bushtit & + & - & + & Yes & $\begin{array}{c}\text { development, wetland, -water, } \\
\text {-urban }\end{array}$ & 0 & 0 & + & No & development, marsh \\
\hline Carolina Wren & + & 0 & 0 & No & - shrub & + & - & & & development, -urban \\
\hline Cassin's Finch & 0 & 0 & + & No & & 0 & - & & & \\
\hline Cassin's Vireo & - & 0 & - & Yes & & - & + & & & \\
\hline Cedar Waxwing & + & + & - & Opposite & wetland & + & + & & & \\
\hline Chipping Sparrow & - & + & $0^{5},-{ }^{9}$ & Partial & & 0 & + & 0 & Yes & \\
\hline Common Yellowthroat & + & 0 & & & wetland & 0 & 0 & & & wetland \\
\hline Dark-eyed Junco & 0 & 0 & 0 & Yes & $\begin{array}{c}\text {-development, -wetland, } \\
\text {-grassland }\end{array}$ & - & 0 & & & water \\
\hline Dusky Flycatcher & - & 0 & - & Yes & & - & 0 & & & \\
\hline Eastern Bluebird & 0 & - & & & & 0 & - & - & No & \\
\hline Eastern Meadowlark & + & - & - & Opposite & & INS & INS & - & & \\
\hline Evening Grosbeak & 0 & + & 0 & Yes & & + & - & & & \\
\hline Golden-crowned Kinglet & 0 & + & 0 & Yes & & 0 & 0 & & & water \\
\hline Golden-cheeked Warbler & INS & INS & & & & INS & INS & 0 & & \\
\hline Gray Flycatcher & - & + & - & Yes & & + & - & + & Yes & \\
\hline Great Crested Flycatcher & - & 0 & 0 & No & development & 0 & + & & & \\
\hline Gray Catbird & 0 & + & & & $\begin{array}{c}\text { development, wetland, -barren, } \\
\text { water }\end{array}$ & 0 & + & & & development \\
\hline Hammond's Flycatcher & 0 & + & & & & - & + & & & \\
\hline Hermit Thrush & - & + & & & & - & 0 & & & \\
\hline House Finch & + & 0 & + & Yes & $\begin{array}{l}\text { development, wetland, shrub, } \\
\text {-barren, grassland }\end{array}$ & 0 & + & + & No & \\
\hline House Sparrow & 0 & 0 & & & development & 0 & + & $0^{10},-{ }^{13}$ & Partial & \\
\hline House Wren & 0 & 0 & & & wetland, -barren & + & 0 & & & wetland, -urban \\
\hline Indigo Bunting & 0 & + & & & & 0 & + & & & -wetland \\
\hline Lazuli Bunting & 0 & 0 & 0 & Yes & shrub, -barren, -marsh & 0 & + & & & -grassland \\
\hline Least Flycatcher & - & 0 & - & Yes & & 0 & 0 & & & \\
\hline Lincoln's Sparrow & - & 0 & & & & - & 0 & & & \\
\hline MacGillivray's Warbler & 0 & + & 0 & Yes & -development, -wetland & + & 0 & & & \\
\hline Magnolia Warbler & - & 0 & & & & 0 & 0 & & & -development, wetland \\
\hline Mountain Chickadee & 0 & + & - & No & marsh, water & 0 & 0 & & & development, marsh \\
\hline Mourning Dove & 0 & 0 & - & No & development & 0 & 0 & - & No & \\
\hline Mrytle Warbler & - & 0 & - & Yes & & - & + & & & development \\
\hline Nashville Warbler & - & 0 & & & -wetland, marsh & - & + & & & \\
\hline Northern Cardinal & 0 & - & & & development & + & 0 & & & \\
\hline Orange-crowned Warbler & + & - & & & & 0 & + & & & \\
\hline Ovenbird & - & + & $0^{1}, 0^{6}$ & No & -wetland & 0 & + & & & \\
\hline Pine Siskin & 0 & 0 & 0 & Yes & $\begin{array}{c}\text { development, -wetland, barren, } \\
\text { urban }\end{array}$ & - & 0 & & & \\
\hline Red-breasted Nuthatch & - & + & $-{ }^{9},-{ }^{12}$ & Yes & marsh & 0 & + & & & \\
\hline Red-eyed Vireo & - & + & $-{ }^{1}, 0^{12}$ & Partial & -development, marsh & + & + & & & \\
\hline
\end{tabular}




\begin{tabular}{|c|c|c|c|c|c|c|c|c|c|c|}
\hline Ruby-crowned Kinglet & + & 0 & - & Opposite & & 0 & 0 & & & \\
\hline Rufous Hummingbird & 0 & 0 & & & & - & - & & & wetland, -grassland \\
\hline Scarlet Tanager & - & - & 0 & No & & 0 & 0 & & & \\
\hline Song Sparrow & 0 & + & & & development, wetland & 0 & 0 & & & marsh \\
\hline Spotted Towhee & 0 & 0 & - & No & -barren, -marsh & 0 & 0 & & & barren, marsh \\
\hline Swainson's Thrush & 0 & 0 & & & & + & 0 & & & wetland, - urban \\
\hline Tennessee Warbler & INS & INS & 0 & & & INS & INS & & & \\
\hline Townsend's Solitaire & 0 & + & 0 & Yes & urban & - & + & & & \\
\hline Townsend's Warbler & 0 & + & - & No & & - & + & & & \\
\hline Warbling Vireo & - & + & & & -wetland & 0 & 0 & & & \\
\hline Western Bluebird & 0 & - & & & development, -grassland & + & 0 & 0 & No & -grassland \\
\hline Western Scrub Jay & + & + & - & Opposite & shrub, - barren & 0 & + & & & \\
\hline Western Tanager & 0 & + & $-{ }^{5},-{ }^{9},-{ }^{12}$ & No & -development & + & 0 & -5 & Opposite & \\
\hline White-breasted Nuthatch & + & 0 & - & Opposite & & - & 0 & & & \\
\hline White-crowned Sparrow & 0 & + & 0 & Yes & & INS & INS & - & & \\
\hline White-eyed Vireo & + & 0 & & & -water & - & + & & & shrub \\
\hline White-throated Sparrow & 0 & - & $-{ }^{1}, 0^{12}$ & Partial & marsh & 0 & + & & & \\
\hline Wilson's Warbler & - & + & & & & 0 & - & & & $\begin{array}{c}\text {-development, -shrub, } \\
\text {-urban }\end{array}$ \\
\hline Wood Thrush & + & 0 & 0 & No & -grassland & + & 0 & & & \\
\hline Wrentit & 0 & 0 & & & $\begin{array}{l}\text { development, wetland, shrub, } \\
\text { barren, marsh, - grassland }\end{array}$ & 0 & - & & & water \\
\hline Yellow Warbler & + & 0 & $-{ }^{9}, 0^{12}$ & No & wetland & 0 & 0 & & & water \\
\hline Yellow-billed Cuckoo & 0 & + & - & No & wetland, shrub & + & 0 & & & \\
\hline Yellow-breasted Chat & - & + & & & & 0 & 0 & & & -shrub \\
\hline
\end{tabular}

\section{Comparison of continental and local studies}

Results from local studies were only compared to our continental results for the weighted mean of noise because each local study used the mean to quantify noise, rather than a measure of spatial heterogeneity. For abundance, data at both scales was available for 51 cases, which included 43 of the 44 available unique species (one continental model failed) and eight cases where multiple local studies were available on a species (two studies in six species, three in one species; Table 1). The directional effect of noise across multiple local studies was congruent for three of these species $(42.9 \%)$. The directional effects of noise on abundance were congruent between scales in 22 of 51 comparisons $(43.1 \%)$, although the trend was reversed (i.e., + to - or vice versa) in only seven studies (13.7\%). One local, multispecies study assessed noise impacts during migration (McClure et al. 2013), a time period that may exert different pressures than during the breeding season when MAPS data was collected. However, removing this study did not improve agreement (10 of 29 cases; $34.5 \%$ ).

For productivity, data at both scales was available for 14 cases, which included 13 of the 16 available unique species (three continental models failed) and one case where an additional local study was available on a species (Table 1). The directional effect of noise between studies was not congruent in this species. The directional effects of noise on productivity between spatial scales was congruent in only 3 of 14 cases $(21.4 \%)$, although the trend was reversed for only two species $(14.2 \%)$.

\section{DISCUSSION}

Changes in abundance associated with mean noise levels predicted from the NPS noise model were detected in 38 of $70(54.2 \%)$ of the species analyzed with MAPS banding data, being distributed equally between positive and negative relationships. Published, localized studies detected noise-associated impacts on species abundance in $58.8 \%(30 / 51)$ of species. Our results indicate that MAPS banding station data detects noise-associated changes in abundance at a similar rate to localized studies and is a useful tool for assessing the impacts of noise on songbird abundance. Fewer noise-associated changes were detected for productivity $(30 / 68$ [44.2\%] compared to $12 / 16[75.0 \%]$ locally). This likely stems from the fact that productivity is impacted by multiple reproductive variables, e.g., fecundity, number of broods, or postfledging survival. Publication skew could also contribute, because local studies are more likely to be conducted on populations where a trend is suspected a priori (Møller and Jennions 2001). It is also plausible that noise is not related to productivity in the majority of species at the continental scale because lowered abundance in noisy areas reduces competition for habitat and foraging resources (Francis et al. 2011). As a result, smaller populations may continue to be proportionally as productive as larger populations. However, if productivity does not increase when abundance decreases, it is likely that populations in noisy areas will remain small or decrease in size over time. Of the 20 species that became less common as mean noise levels increased, only two also had positive productivity (Gray Flycatcher [Empidonax wrightii] and Red-eyed Vireo [Vireo olivaceus]).

Perhaps one of the primary benefits of MAPS banding data is the ability to compare noise-associated changes in abundance and productivity simultaneously across a large number of sites and years. In our results, the relationship with noise in one population parameter did not consistently predict the direction of the other. In general, ecologists do not expect abundance and productivity to be correlated, largely because of density-dependent processes (Van Horne 1983, Murdoch 1994). However, in the presence of an external stressor, such as noise or urbanization, it is plausible that both processes could be reduced in parallel by a reduction in fitness that directly impacts habitat selection and the production of offspring simultaneously (Bock and Jones 2004). Our results indicate that the interaction between noise and population demographics is more complex, and that monitoring long-term changes using both measures in tandem might be the most useful for predicting population persistence in noisy areas.

For example, abundance and productivity both increased with mean noise level across MAPS stations in four species: the American Robin (Turdus migratorius), Carolina Wren 
(Thryothorus ludovicianus), Cedar Waxwing (Bombycilla cedrorum), and Wood Thrush (Hylocichla mustelina). Although Wood Thrush populations have declined generally (Rushing et al. 2016), all of these species are common in human-dominated systems in North America, and their ability to capitalize on noisy habitats vacated by many other species may partially underlie their synanthropic success (e.g., Goodwin and Shriver 2011). Conversely, because abundance and productivity both declined as noise level increased, we might predict that the American Redstart, Cassin's Vireo (Vireo cassinii), Dusky Flycatcher (Empidonax oberholseri), Hermit Thrush (Catharus guttatus), Lincoln's Sparrow (Melospiza lincolnii), Yellow-rumped Warbler (Setophaga coronata), and Nashville Warbler (Leiothlypis ruficapilla) will decline if noise continues to become more pervasive. Species such as the American Goldfinch (Spinus tristis), Black-capped Chickadee (Poecile atricapillus), and House Finch (Haemorhous mexicanus) might increase gradually because their abundance increased in noise, while productivity was unaffected. However, species like the Chipping Sparrow (Spizella passerina), Great Crested Flycatcher, (Myiarchus crinitus), and Least Flycatcher (Empidonax minimus) might slowly decrease because of declining abundance in noise, even though productivity is stable.

In addition to providing multiple demographic measures, data from banding stations is unaffected by some of the confounding variables that typically plague noise studies. Specifically, banding data is robust to the problem of observer detectability. Variation in aural abilities between observers can limit comparisons from aural surveys, a primary method for assessing abundance in avian studies (Faanes and Bystrak 1981). Ambient noise level is also known to impact detectability (Simons et al. 2009), making it difficult to compare data across studies unless similar methodology allows for statistical corrections (e.g., Buckland et al. 1993). Barring the presence of a point source of noise physically located near a mist net, there is little reason to believe that ambient noise has any impact on the likelihood of capturing resident birds that are present on the breeding or winter grounds. Further, having a bird in hand, where photographs, previous bands, and expert help can be utilized, reduces error and variability in identification and counts between observers.

Several studies have investigated whether particular life history traits underlie differential species responses to noise. In our analysis, neither acoustic traits, nor a suite of guilds defined by life history traits were predictive. The lack of any effect was somewhat surprising given recent literature on the subject that has implicated vocal frequency, diet, and nesting as traits related to noise responses (Hu and Cardoso 2009, Cardoso 2014, Francis 2015). However, in each of these published studies, life history traits were compiled for several hundred species, often selected to cover a range of variation in vocal features and life history traits. Despite their extent, some results from these large-scale studies are still conflicting (Moiron et al. 2015). Our study included 72 species that were selected because they were commonly captured in mist nests at MAPS stations or because continental data could be compared to data from local studies. Because our primary objective was to evaluate the viability of detecting noiseassociated changes in MAPS data, we made no attempt to capture a range of vocal or life history traits. Thus, variation in some traits was low. For example, only four species of grassland birds were included in our analysis, and very few of our species were not insectivores. Further, noise in our study was not constricted to low-frequency sources, which may reduce the role of vocal frequency often seen in urban adaptation (Halfwerk and Slabbekoorn 2009). Although a more explicit study of life history traits across species captured at MAPS stations could be valuable, we suggest that our results should be limited to evaluating the contribution of MAPS data for detecting noise-associated patterns in bird abundance and productivity.

Although mean noise level is the most common unit used to assess the impacts of noise, spatially heterogeneous acoustic habitats may impact birds differently (Gill et al. 2015). Acoustic niches, for example, could be more diverse when noise levels vary across space. As a result, birds with both philic and phobic reactions to noise might inhabit the same general area. In keeping with this hypothesis, $85.7 \%$ of species increased or remained stable as noise heterogeneity increased, while only $72.9 \%$ did so as mean noise level increased. Although our effects sizes are small, the skew toward more positive relationships with noise heterogeneity (Figs. 2 and 3) may provide an acoustic example of the intermediate disturbance hypothesis, which predicts that the highest number of species will thrive when habitats are neither uniformly pristine nor highly degraded (Connell 1978). At minimum, the negative relationship with spatially heterogeneous noise is less prominent than that of a uniform increase in noise. This suggests that banding stations with elevated noise levels on only a portion of their localized habitats may support more diverse songbird communities than those completely inundated by elevated noise levels. Perhaps this is not surprising given that localized noise sources, such as rivers, have been on the landscape for much of geologic history. Another possibility is that these communities are experiencing a release from predation by noise averse predators (Francis et al. 2009, Barber et al. 2010), without incurring the full costs associated with uniformly increased noise levels. Clearly, a better understanding of the spatial extent at which noise impacts songbirds is needed. More generally, studies of animal ecology must continue to move away from interpretations based solely on averages. Variability in many sensory cues, habitat characteristics, temporal patterns, and even life-history traits may impact populations differently.

Combining results from the continental-scale MAPS banding database with localized studies may also be important for interpreting noise impacts. Congruent results from the two spatial extents might provide confidence for widespread species-specific management techniques. One example is the Red-breasted Nuthatch (Sitta canadensis), where abundance was negatively associated with noise in two localized studies and in our continental assessment. Taken together, these results indicate that noise mitigation will likely be needed to maintain healthy population levels in this species. Conversely, the abundance of the Western Tanager (Piranga ludoviciana) was negatively impacted in multiple regional studies, but not in our continental model. In this case, the fine-grain detail of localized studies may have detected patterns not present or detectable in MAPS data, and localized or general noise-mitigation might still be recommended for species management. Finally, the MAPS dataset detected no relationship between noise and productivity in House Sparrows, a pattern that is supported in one localized study (Meillère et al. 2015), but not in another (Schroeder et al. 2012). The lack of an 
outcome at the larger spatial extent might reduce concern that noise is impacting this species, despite the more variable localized results.

Determining the impact of noise on populations dynamics in particular species is critical as we prioritize mitigation objectives for songbirds. Extrapolating solely from local studies may lead to misguided management because of the presence of differential local and landscape level processes. Given that broad metaanalyses to date have been unable to consistently predict noise impacts based on a single set of life-history traits, large scale datasets capable of determining patterns of change directly for individual species may be a valuable tool for assessing noise impacts. Our results suggest that MAPS data can be used to detect noise-related changes at the continental level. The value of this dataset is enhanced by the ability to incorporate habitat and development variables alongside noise terms to assess patterns in multiple population parameters, including, abundance, productivity, and perhaps eventually survivorship. In our models, over a quarter of the species surveyed experienced declines in abundance or productivity beyond what could be attributed to other variables, highlighting the importance of noise mitigation for maintaining songbird diversity. Because the direction of noiseassociated changes was not correlated between abundance and productivity, and continental and localized results were not consistently congruent, best management practices should strive to incorporate multiple demographic measurements from multiple scales. Agreement across scales and population parameters may reveal which species are most and least sensitive to noise. Future attempts to model noise over time, or take standardized noise measurements at MAPS stations, might increase the sensitivity of this dataset. However, the current MAPS dataset can be used to derive continental, noise-associated trends for any bird species that is regularly captured in mist nets, adding a new tool for biologists seeking to understand and manage songbird populations in an age of increasing noise levels.

\section{Data Availability Statement}

Data (allspecies_final.csv) that supports the findings of this study is available online in the OSF public repository (https://osf.io/ cka9z/). $\mathrm{R}$ code for single species model analysis is available in Appendix 6 and code for the measurement error models is in Appendix 7.

Responses to this article can be read online at: http://www.ace-eco.org/issues/responses.php/1633

\section{Acknowledgments:}

We would like to thank D. DeSante, S. Albert, and D. Kaschube from the Institute of Bird Populations for providing access to the MAPS database. We are also indebted to the National Park Service Natural Sounds and Night Sky Division for developing and providing access to the georeferenced CONUS noise map. We thank M. Link for discussions and data analysis that contributed to an earlier version of this work. Finally, we are grateful for the helpful feedback provided by the editors and two anonymous reviewers that greatly improved the manuscript. The authors have no conflict of interests to report.

\section{LITERATURE CITED}

Barber, J. R., K. R. Crooks, and K. M. Fristrup. 2010. The costs of chronic noise exposure for terrestrial organisms. Trends in Ecology \& Evolution 25:180-189. https://doi.org/10.1016/j. tree.2009.08.002

Bayne, E. M., L. Habib, and S. Boutin. 2008. Impacts of chronic anthropogenic noise from energy-sector activity on abundance of songbirds in the boreal forest. Conservation Biology 22:1186-1193. https://doi.org/10.1111/j.1523-1739.2008.00973.x

Bock, C. E., and Z. F. Jones. 2004. Avian habitat evaluation: should counting birds count? Frontiers in Ecology and the Environment 2:403-410. https://doi.org/10.1890/1540-9295(2004) 002[0403:ahescb]2.0.co;2

Buckland, S. T., D. R. Anderson, K. P. Burnham, and J. L. Laake. 1993. Distance sampling: estimating abundance of biological populations. Chapman \& Hall, London, UK.

Bürkner, P.-C. 2018. Advanced Bayesian multilevel modeling with the $\mathrm{R}$ package brms. $R$ Journal 10:395-411. https://doi. org/10.32614/RJ-2018-017

Burnham, K. P., and D. R. Anderson. 2002. Model selection and multimodel inference. Springer Verlag, New York, New York, USA. https://doi.org/10.1007/b97636

Burnham, K. P., and D. R. Anderson. 2004. Multimodel inference: understanding AIC and BIC in model selection. Sociological Methods and Research 33(2):261-304. https://doi. org/10.1177/0049124104268644

Buxton, R. T., M. F. McKenna, D. Mennitt, K. Fristrup, K. Crooks, L. Angeloni, and G. Wittemyer. 2017. Noise pollution is pervasive in U.S. protected areas. Science 356:531-533. https://doi. org/10.1126/science.aah4783

Cardoso, G. C. 2014. Nesting and acoustic ecology, but not phylogeny, influence passerine urban tolerance. Global Change Biology 20:803-810. https://doi.org/10.1111/gcb.12410

Cardoso, G. C., Y. Hu, and C. D. Francis. 2018. The comparative evidence for urban species sorting by anthropogenic noise. Royal Society Open Science 5:172059. https://doi.org/10.1098/ rsos. 172059

Connell, J. H. 1978. Diversity in tropical rain forests and coral reefs. Science 199:1302-1310. https://doi.org/10.1126/ science.199.4335.1302

Cuddington, K. 2011. Legacy effects: the persistent impact of ecological interactions. Biological Theory 6:203-210. https://doi. org/10.1007/s13752-012-0027-5

DeSante, D. F., and D. R. Kaschube. 2009. The monitoring avian productivity and survivorship (MAPS) program 2004, 2005, and 2006 report. Bird Populations 9:86-169.

DeSante, D. F., D. R. Kaschube, and J. F. Saracco. 2015. Vital rates of North American landbirds. The Institute for Bird Populations, Petaluma, California, USA.

Dormann, C. F., J. Elith, S. Bacher, C. Buchmann, G. Carl, G. Carré, J. R. G. Marquéz, B. Gruber, B. Lafourcade, P. J. Leitão, T. Münkemüller, C. McClean, P. E. Osborne, B. Reineking, B. Schröder, A. K. Skidmore, D. Zurell, and S. Lautenbach. 2013. Collinearity: a review of methods to deal with it and a simulation 
study evaluating their performance. Ecography 36:27-46. https:// doi.org/10.1111/j.1600-0587.2012.07348.x

Dunn, E. H., and C. J. Ralph. 2004. The use of mist nets as a tool for bird population monitoring. Studies in Avian Biology 29:1-6.

Eglington, S. M., R. Julliard, G. Gargallo, H. P. van der Jeugd, J. W. Pearce-Higgins, S. R. Baillie, and R. A. Robinson. 2015. Latitudinal gradients in the productivity of European migrant warblers have not shifted northwards during a period of climate change. Global Ecology and Biogeography 24:427-436. https://doi. org/10.1111/geb.12267

Faanes, C. A., and D. Bystrak. 1981. The role of observer bias in the North American Breeding Bird Survey. Studies in Avian Biology 6:353-359.

Francis, C. D. 2015. Vocal traits and diet explain avian sensitivities to anthropogenic noise. Global Change Biology 21:1809-1820. https://doi.org/10.1111/gcb.12862

Francis, C. D., C. P. Ortega, and A. Cruz. 2009. Noise pollution changes avian communities and species interactions. Current Biology 19:1415-1419. https://doi.org/10.1016/j.cub.2009.06.052

Francis, C. D., J. Paritsis, C. P. Ortega, and A. Cruz. 2011. Landscape patterns of avian habitat use and nest success are affected by chronic gas well compressor noise. Landscape Ecology 26:1269-1280. https://doi.org/10.1007/s10980-011-9609-Z

Gill, S. A., J. R. Job, K. Myers, K. Naghshineh, and M. J. Vonhof. 2015. Toward a broader characterization of anthropogenic noise and its effects on wildlife. Behavioral Ecology 26:328-333. https:// doi.org/10.1093/beheco/aru219

Goodwin, S. E., and W. G. Shriver. 2011. Effects of traffic noise on occupancy patterns of forest birds. Conservation Biology 25:406-411. https://doi.org/10.1111/j.1523-1739.2010.01602.x

Habib, L., E. M. Bayne, and S. Boutin. 2007. Chronic industrial noise affects pairing success and age structure of ovenbirds Seiurus aurocapilla. Journal of Applied Ecology 44:176-184. https://doi.org/10.1111/j.1365-2664.2006.01234.x

Haldane, J. B. S. 1956. The relation between density regulation and natural selection. Proceedings of the Royal Society of London B: Biological Sciences 145:306-308. https://doi.org/10.1098/ rspb.1956.0039

Halfwerk, W., C. Both, and H. Slabbekoorn. 2016. Noise affects nest-box choice of 2 competing songbird species, but not their reproduction. Behavioral Ecology 27:1592-1600. https://doi. org/10.1093/beheco/arw095

Halfwerk, W., L. J. M. Holleman, C. M. Lessells, and H. Slabbekoorn. 2011. Negative impact of traffic noise on avian reproductive success. Journal of Applied Ecology 48:210-219. https://doi.org/10.1111/j.1365-2664.2010.01914.x

Halfwerk, W., and H. Slabbekoorn. 2009. A behavioural mechanism explaining noise-dependent frequency use in urban birdsong. Animal Behaviour 78:1301-1307. https://doi.org/10.1016/ j.anbehav.2009.09.015

Hastie, T. J., and R. J. Tibshirani. 1990. Generalized additive models. Chapman \& Hall/CRC Press, Boca Raton, Florida, USA.
Homer, C., J. Dewitz, L. Yang, S. Jin, P. Danielson, G. Xian, J. Coulston, N. Herold, J. Wickham, and K. Megown. 2015. Completion of the 2011 National land cover database for the conterminous United States - representing a decade of land cover change information. Photogrammetric Engineering \& Remote Sensing 81:345-354.

Homer, C. H., J. A. Fry, and C. A. Barnes. 2012. The National Land Cover Database, U.S. Geological Survey Fact Sheet 2012-3020. U.S. Department of the Interior, Washington, D.C., USA.

Hu, Y., and G. C. Cardoso. 2009. Are bird species that vocalize at higher frequencies preadapted to inhabit noisy urban areas? Behavioral Ecology 20:1268-1273. https://doi.org/10.1093/ beheco/arp131

Khan, I., S. U. Khan, M. Zhao, and A. A. Khan. 2019. Exploring the spatial heterogeneity of individual preferences for integrated river basin management: an example of Heihe river basin. Environmental Science and Pollution Research 26:6911-6921. https://doi.org/10.1007/s11356-019-04178-2

Kleist, N. J., R. P. Guralnick, A. Cruz, and C. D. Francis. 2017. Sound settlement: noise surpasses land cover in explaining breeding habitat selection of secondary cavity-nesting birds. Ecological Applications 27:260-273. https://doi.org/10.1002/ eap. 1437

McClure, C. J. W., A. C. Korte, J. A. Heath, and J. R. Barber. 2015. Pavement and riparian forest shape the bird community along an urban river corridor. Global Ecology and Conservation 4:291-310. https://doi.org/10.1016/j.gecco.2015.07.004

McClure, C. J. W., H. E. Ware, J. D. Carlisle, and J. R. Barber. 2017. Noise from a phantom road experiment alters the age structure of a community of migrating birds. Animal Conservation 20:164-172. https://doi.org/10.1111/acv.12302

McClure, C. J., H. E. Ware, J. Carlisle, G. Kaltenecker, and J. R. Barber. 2013. An experimental investigation into the effects of traffic noise on distributions of birds: avoiding the phantom road. Proceedings of the Royal Society of London B: Biological Sciences 280:20132290. https://doi.org/10.1098/rspb.2013.2290

McElreath, R. 2020. Statistical rethinking: a Bayesian course with examples in R and Stan. CRC Press/Taylor \& Francis Group, Boca Raton, Florida, USA.

Meillère, A., F. Brischoux, C. Parenteau, and F. Angelier. 2015. Influence of urbanization on body size, condition, and physiology in an urban exploiter: a multi-component approach. PLOS ONE 10:e0135685. https://doi.org/10.1371/journal.pone.0135685

Melles, S., S. Glenn, and K. Martin. 2003. Urban bird diversity and landscape complexity: species-environment associations along a multiscale habitat gradient. Conservation Ecology 7(1):5. https://doi.org/10.5751/ES-00478-070105

Mennitt, D., K. Sherrill, and K. Fristrup. 2014. A geospatial model of ambient sound pressure levels in the contiguous United States. Journal of the Acoustical Society of America 135 (5):2746-2764. https://doi.org/10.1121/1.4870481

Moiron, M., C. González-Lagos, H. Slabbekoorn, and D. Sol. 2015. Singing in the city: high song frequencies are no guarantee 
for urban success in birds. Behavioral Ecology 26:843-850. https:// doi.org/10.1093/beheco/arv026

Møller, A. P., and M. D. Jennions. 2001. Testing and adjusting for publication bias. Trends in Ecology \& Evolution 16:580-586. https://doi.org/10.1016/S0169-5347(01)02235-2

Murdoch, W. W. 1994. Population regulation in theory and practice. Ecology 75:271-287. https://doi.org/10.2307/1939533

Piironen, J., M. Paasiniemi, A. Vehtari, J. Gabry, P.-C. Bürkner, and M. Colombo. 2019. projpred: Projection predictive feature selection. $\mathrm{R}$ package.

Piironen, J., and A. Vehtari. 2017. Sparsity information and regularization in the horseshoe and other shrinkage priors. Electronic Journal of Statistics 11:5018-5051. https://doi. org/10.1214/17-EJS1337SI

Proppe, D. S., C. B. Sturdy, and C. C. St Clair. 2013. Anthropogenic noise decreases urban songbird diversity and may contribute to homogenization. Global Change Biology 19:1075-1084. https://doi.org/10.1111/gcb.12098

Pulliam, H. R. 1988. Sources, sinks, and population regulation. American Naturalist 132:652-661. https://doi.org/10.1086/284880

R Core Team. 2019. R: A language and environment for statistical computing. R Foundation for Statistical Computing, Vienna, Austria. [online] URL: https://www.R-project.org/

Rahbek, C. 2005. The role of spatial scale and the perception of large-scale species-richness patterns. Ecology Letters 8:224-239. https://doi.org/10.1111/j.1461-0248.2004.00701.x

Reijnen, R., and R. Foppen. 1995. The effects of car traffic on breeding bird populations in woodland. IV. Influence of population size on the reduction of density close to a highway. Journal of Applied Ecology 32:481-491. https://doi.org/10.2307/2404646

Rushing, C. S., T. B. Ryder, and P. P. Marra. 2016. Quantifying drivers of population dynamics for a migratory bird throughout the annual cycle. Proceedings of the Royal Society B: Biological Sciences 283(1823):20152846. https://doi.org/10.1098/rspb.2015.2846

Sallabanks, R., E. B. Arnett, and J. M. Marzluff. 2000. An evaluation of research on the effects of timber harvest on bird populations. Wildlife Society Bulletin (1973-2006) 28:1144-1155.

Schoener, T. W. 1983. Field experiments on interspecific competition. American Naturalist 122:240-285. https://doi. org/10.1086/284133

Schroeder, J., S. Nakagawa, I. R. Cleasby, and T. Burke. 2012. Passerine birds breeding under chronic noise experience reduced fitness. PLoS ONE 7:e39200. https://doi.org/10.1371/journal. pone. 0039200

Shannon, G., M. F. McKenna, L. M. Angeloni, K. R. Crooks, K. M. Fristrup, E. Brown, K. A. Warner, M. D. Nelson, C. White, J.
Briggs, S. McFarland, and G. Wittemyer. 2016. A synthesis of two decades of research documenting the effects of noise on wildlife. Biological Reviews 91:982-1005. https://doi.org/10.1111/ brv. 12207

Sherry, T. W., and R. T. Holmes. 1988. Habitat selection by breeding American Redstarts in response to a dominant competitor, the Least Flycatcher. Auk 105:350-364. https://doi. org/10.2307/4087501

Simons, T. R., K. H. Pollock, J. M. Wettroth, M. W. Alldredge, K. Pacifici, and J. Brewster. 2009. Sources of measurement error, misclassification error, and bias in auditory avian point count data. Pages 237-254 in D. L. Thomson, E. G. Cooch, and M. J. Conroy, editors. Modeling demographic processes in marked populations. Springer, New York, New York, USA. https://doi. org/10.1007/978-0-387-78151-8_10

Slabbekoorn, H., and M. Peet. 2003. Ecology: birds sing at a higher pitch in urban noise. Nature 424:267. https://doi. org/10.1038/424267a

Van Horne, B. 1983. Density as a misleading indicator of habitat quality. Journal of Wildlife Management 47:893-901. https://doi. org/10.2307/3808148

Vehtari, A., A. Gelman, and J. S. Gabry. 2017. Practical Bayesian model evaluation using leave-one-out cross-validation and WAIC. Statistics and Computing 27:1413-1432. https://doi. org/10.1007/s11222-016-9696-4

Wood, S. N. 2011. Fast stable restricted maximum likelihood and marginal likelihood estimation of semiparametric generalized linear models. Journal of the Royal Statistical Society (B) 73 (1):3-36. https://doi.org/10.1111/j.1467-9868.2010.00749.x

Wood, S. N. 2017. Generalized additive models: an introduction with R. CRC Press/Taylor \& Francis Group, Boca Raton, Florida, USA.

Yang, Z., X. Liu, M. Zhou, D. Ai, G. Wang, Y. Wang, C. Chu, and J. T. Lundholm. 2015. The effect of environmental heterogeneity on species richness depends on community position along the environmental gradient. Scientific Reports 5:15723. https://doi.org/10.1038/srep15723

Zuur, A. F., E. N. Ieno, N. Walker, A. A. Saveliev, and G. M. Smith. 2009. Mixed effects models and extensions in ecology with R. Springer Verlag, New York, New York, USA. https://doi. org/10.1007/978-0-387-87458-6
Editor-in-Chief: Alexander L.Bond

Subject Editor: Erin Bayne
Sponsored by the Society of Canadian Ornithologists and Birds Canada Parrainée par la Société des ornithologistes du Canada et Oiseaux Canada

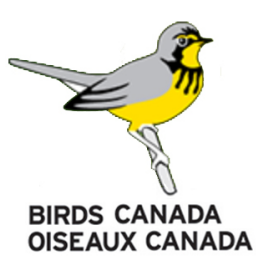


Appendix 1 | Regional studies selected for continental comparisons

${ }^{1}$ Bayne et al. 2008, Conservation Biology

Species

Ovenbird

Red-Eyed Vireo

Tennessee Warbler

White-Throated Sparrow

Yellow-rumped Warbler

2 Dietz et al. 2013, The Wilson Journal of Ornithology

${ }^{3}$ Forman et al. 2002, Environmental Management

White-crowned Sparrow

Bobolink

${ }^{4}$ Francis et al. 2011, Landscape Ecology

${ }^{5}$ Francis et al. 2011, Plos One

${ }^{6}$ Goodwin and Shriver 2011, Conservation Biology

${ }^{7}$ Kight and Swaddle 2012, Ecological Applications

${ }^{8}$ Lackey et al, 2012, Ornithological Monographs

${ }^{9}$ McClure et al. 2013, Proceedings of the Royal Society $B$

${ }^{10}$ Meillere et al. 2015, Biology Letters

${ }^{11}$ Mulholland 2016 - Dissertation Cal Poly

12 Proppe et al. 2013, Global Change BiologyAppen

${ }^{13}$ Schroeder et al. 2012, Plos One

† Superscripts correspond with notations in Table 1

Easten Meadow Lark

Gray Flycatcher

Western Scrub-Jay

Black-headed Grosbeak

Black-Chinned Hummingbird

Bushtit

Chipping Sparrow

House Finch

Mourning Dove

Western Tanager

Acadian Flycatcher

Carolina Wren

Great Crested Flycatcher

Ovenbird

Scarlet Tanager

White Breasted Nuthatch

Wood Thrush

Yellow-Billed Cuckoo

Eastern Bluebird

Golden-Cheeked Warbler

American Robin

Cassin's Finch

Cassin's Vireo

Cedar Waxwing

Chipping Sparrow

Dark-eyed Junco

Dusky Flycatcher

Evening Grosbeak

Golden Crowned Kinglet

Lazuli Bunting

Macgillivray's Warbler

Mountain Chickadee

Pine Sisken

Red-Breasted Nuthatch

Ruby-Crowned Kinglet

Spotted Towhee

Townsend's Solitaire

Townsend's Warbler

Western Tanager

White-crowned Sparrow

Yellow Warbler

Yellow-rumped Warbler

House Sparrow

Western Bluebird

Ash-throated Flycatcher

Black-capped Chickadee

Least Flycatcher

Red-Breasted Nuthatch

Red-Eyed Vireo

Western Tanager

White-Throated Sparrow

Yellow Warbler

House Sparrow
Demographic measure Directional effect of noise

Abundance None

Abundance Negative

Abundance None

Abundance Negative

Abundance Negative

Productivity Negative

Abundance, Productivity Negative

Abundance, Productivity Negative

Abundance, Productivity Negative, Postive

Abundance

Abundance, Productivity Positive

Abundance, Productivity Positive

Abundance, Productivity Neutral

Abundance, Productivity Positive

Abundance, Productivity Negative

Abundance, Productivity Negative

Abundance None

Abundance None

Abundance None

Abundance None

Abundance None

Abundance Negative

Abundance None

Abundance Negative

Productivity Negative

Productivity None

Abundance Negative

Abundance Positive

Abundance Negative

Abundance Negative

Abundance Negative

Abundance Neutral

Abundance Negative

Abandance Neutral

Abandance Neutral

Abundance Neutral

Abundance Neutral

Abundance Negative

Abundance Neutral

Abundance Negative

Abundance Negative

Abundance Negative

Abundance Neutral

Abundance Negative

Abundance Negative

Abundance Neutral

Abundance Negative

Abundance Neutral

Productivity None

Productivity None

Productivity Negative

Abundance None

Abundance Negative

Abundance Negative

Abundance None

Abundance Negative

Abundance None

Abundance None

Productivity Negative 
Appendix 2 | Results from principal components analysis. Component names are deduced from up to three of the strongest contributors, which are bolded.

\begin{tabular}{|c|c|c|c|c|c|c|c|c|c|c|c|}
\hline & PC1 & PC2 & PC3 & PC4 & PC5 & PC6 & PC7 & PC8 & PC9 & PC10 & PC11 \\
\hline Percent variance explained & 20.08 & 11.75 & 10.78 & 9.22 & 8.15 & 7.49 & 7.46 & 6.84 & 6.37 & 6.02 & 5.85 \\
\hline Cumulative variance explained & 20.08 & 31.83 & 42.60 & 51.82 & 59.97 & 67.47 & 74.92 & 81.77 & 88.14 & 94.15 & 100.00 \\
\hline Dominant terms & Development & Wetland & Shrub & Barren & Marsh & Grassland & Water & Urban & NA & NA & NA \\
\hline Developed: Open/Low & 0.60 & -0.34 & 0.04 & 0.05 & 0.00 & 0.04 & -0.03 & -0.72 & NA & NA & NA \\
\hline Developed: Mid/High & 0.59 & -0.33 & 0.08 & 0.14 & 0.03 & -0.10 & -0.07 & 0.61 & NA & NA & NA \\
\hline Cultivated & 0.19 & 0.20 & -0.26 & -0.62 & -0.57 & 0.06 & 0.15 & -0.04 & NA & NA & NA \\
\hline Water & 0.17 & 0.31 & -0.29 & 0.44 & 0.12 & 0.22 & 0.71 & -0.03 & NA & NA & NA \\
\hline Wetlands & 0.16 & 0.51 & -0.30 & 0.04 & 0.41 & -0.27 & -0.47 & -0.16 & NA & NA & NA \\
\hline Herbaceous & 0.06 & 0.15 & 0.46 & -0.24 & 0.33 & 0.73 & -0.08 & -0.01 & NA & NA & NA \\
\hline Barren & 0.01 & 0.21 & 0.05 & 0.55 & -0.60 & 0.32 & -0.43 & -0.01 & NA & NA & NA \\
\hline Shrubland & -0.10 & 0.16 & 0.68 & 0.13 & -0.15 & -0.45 & 0.22 & -0.20 & NA & NA & NA \\
\hline Forest & -0.44 & -0.53 & -0.27 & 0.14 & 0.05 & 0.14 & -0.04 & -0.19 & NA & NA & NA \\
\hline
\end{tabular}




\section{Appendix 3: \\ Tables with best models for individual bird species}

Note: Models for the Golden-cheeked Warbler (Setophaga chrysoparia) and Tennessee Warbler (Leiothlypis peregrina) could not be fitted, as stated in the main text.

\begin{tabular}{lrrrr}
\hline A. parametric coefficients & Estimate & Std. Error & t-value & p-value \\
Intercept & -2.6054 & 0.3132 & -8.3175 & $<0.0001$ \\
Noise Standard Deviation & -0.0160 & 0.2096 & -0.0762 & 0.9393 \\
PC 1 & -0.3465 & 0.2204 & -1.5720 & 0.1162 \\
PC 2 & 0.3556 & 0.1567 & 2.2696 & 0.0234 \\
PC 3 & 0.0741 & 0.2932 & 0.2526 & 0.8006 \\
PC 4 & -0.1644 & 0.1642 & -1.0011 & 0.3169 \\
PC 5 & 0.0868 & 0.1801 & 0.4818 & 0.6300 \\
PC 6 & 0.1050 & 0.3005 & 0.3495 & 0.7268 \\
PC 7 & 0.2130 & 0.1528 & 1.3936 & 0.1637 \\
PC 8 & -0.8047 & 0.3873 & -2.0775 & 0.0380 \\
\hline B. smooth terms & edf & Ref.df & F-value & p-value \\
Location Smooth & 73.4369 & 249.0000 & 0.7489 & $<0.0001$ \\
Year Smooth & 0.0007 & 19.0000 & 0.0000 & 0.3630 \\
Station Random Effect & 0.0001 & 7.0000 & 0.0000 & 0.8370 \\
\hline
\end{tabular}

Table A3.1: Best productivity model for the Acadian Flycatcher (Empidonax virescens). The model was a quasi-Poisson GAMM with log link function. Based on AIC/QIC, support for including the noise level as a predictor of productivity was: none; support for including the standard deviation of noise was: weak. 


\begin{tabular}{lrrrr}
\hline A. parametric coefficients & Estimate & Std. Error & t-value & p-value \\
Intercept & -5.8182 & 0.2129 & -27.3236 & $<0.0001$ \\
PC 1 & 0.1128 & 0.0926 & 1.2189 & 0.2230 \\
PC 2 & 0.1211 & 0.1220 & 0.9933 & 0.3207 \\
PC 3 & -0.0489 & 0.2944 & -0.1660 & 0.8682 \\
PC 4 & -0.1536 & 0.1439 & -1.0678 & 0.2857 \\
PC 5 & 0.3898 & 0.1645 & 2.3701 & 0.0179 \\
PC 6 & 0.4306 & 0.2533 & 1.7002 & 0.0892 \\
PC 7 & -0.2547 & 0.1586 & -1.6061 & 0.1084 \\
PC 8 & 0.1965 & 0.1273 & 1.5433 & 0.1229 \\
\hline B. smooth terms & edf & Ref.df & F-value & p-value \\
Location Smooth & 15.3216 & 19.0000 & 3580.1975 & $<0.0001$ \\
Year Smooth & 4.5580 & 7.0000 & 51.6030 & 0.0057 \\
Station Random Effect & 197.5729 & 257.0000 & 9.0390 & $<0.0001$ \\
\hline
\end{tabular}

Table A3.2: Best abundance model for the Acadian Flycatcher (Empidonax virescens). The model was a quasi-Poisson GAMM with log link function. Based on AIC/QIC, support for including the noise level as a predictor of abundance was: none; support for including the standard deviation of noise was: none.

\begin{tabular}{lrrrr}
\hline A. parametric coefficients & Estimate & Std. Error & t-value & p-value \\
Intercept & -5.4605 & 0.4377 & -12.4746 & $<0.0001$ \\
PC 1 & -0.0294 & 0.2727 & -0.1077 & 0.9142 \\
PC 2 & -0.0476 & 0.3322 & -0.1432 & 0.8861 \\
PC 3 & 0.0263 & 0.3658 & 0.0719 & 0.9427 \\
PC 4 & 0.2151 & 0.3093 & 0.6954 & 0.4869 \\
PC 5 & 0.0735 & 0.3913 & 0.1877 & 0.8511 \\
PC 6 & -0.3887 & 0.3792 & -1.0252 & 0.3054 \\
PC 7 & 0.1133 & 0.3527 & 0.3211 & 0.7482 \\
PC 8 & 0.0440 & 0.4541 & 0.0969 & 0.9228 \\
\hline B. smooth terms & edf & Ref.df & F-value & p-value \\
Location Smooth & 140.6609 & 424.0000 & 0.5737 & $<0.0001$ \\
Year Smooth & 3.8026 & 19.0000 & 123.6690 & 0.0822 \\
Station Random Effect & 5.9106 & 7.0000 & 45.6381 & 0.0585 \\
\hline
\end{tabular}

Table A3.3: Best productivity model for the American Goldfinch (Spinus tristis). The model was a quasi-Poisson GAMM with log link function. Based on AIC/QIC, support for including the noise level as a predictor of productivity was: none; support for including the standard deviation of noise was: none. 


\begin{tabular}{lrrrr}
\hline A. parametric coefficients & Estimate & Std. Error & t-value & p-value \\
Intercept & -6.3278 & 1.1690 & -5.4131 & $<0.0001$ \\
Noise Level & 0.0231 & 0.0304 & 0.7576 & 0.4487 \\
Noise Standard Deviation & -0.1615 & 0.1671 & -0.9661 & 0.3341 \\
PC 1 & 0.1727 & 0.0905 & 1.9072 & 0.0566 \\
PC 2 & 0.2258 & 0.0794 & 2.8441 & 0.0045 \\
PC 3 & 0.1506 & 0.1053 & 1.4303 & 0.1527 \\
PC 4 & -0.2066 & 0.0650 & -3.1805 & 0.0015 \\
PC 5 & -0.2447 & 0.0804 & -3.0427 & 0.0024 \\
PC 6 & 0.1136 & 0.0906 & 1.2540 & 0.2099 \\
PC 7 & 0.1356 & 0.0775 & 1.7487 & 0.0804 \\
PC 8 & -0.0539 & 0.1058 & -0.5090 & 0.6108 \\
\hline B. smooth terms & edf & Ref.df & F-value & p-value \\
Location Smooth & 12.4968 & 19.0000 & 5169.9010 & $<0.0001$ \\
Year Smooth & 5.9262 & 7.0000 & 522.0755 & $<0.0001$ \\
Station Random Effect & 346.3808 & 429.0000 & 12.5333 & $<0.0001$ \\
\hline
\end{tabular}

Table A3.4: Best abundance model for the American Goldfinch (Spinus tristis). The model was a quasi-Poisson GAMM with log link function. Based on AIC/QIC, support for including the noise level as a predictor of abundance was: weak; support for including the standard deviation of noise was: weak.

\begin{tabular}{lrrrr}
\hline A. parametric coefficients & Estimate & Std. Error & t-value & p-value \\
Intercept & 1.2183 & 1.9011 & 0.6409 & 0.5218 \\
Noise Level & -0.0803 & 0.0504 & -1.5927 & 0.1115 \\
PC 1 & 0.0304 & 0.1688 & 0.1798 & 0.8573 \\
PC 2 & -0.2137 & 0.1107 & -1.9302 & 0.0539 \\
PC 3 & -0.0203 & 0.2268 & -0.0894 & 0.9288 \\
PC 4 & 0.0264 & 0.1167 & 0.2266 & 0.8208 \\
PC 5 & 0.1184 & 0.1434 & 0.8254 & 0.4093 \\
PC 6 & 0.0006 & 0.2495 & 0.0026 & 0.9980 \\
PC 7 & 0.1096 & 0.1290 & 0.8493 & 0.3959 \\
PC 8 & 0.2311 & 0.2448 & 0.9441 & 0.3453 \\
\hline B. smooth terms & edf & Ref.df & F-value & p-value \\
Location Smooth & 71.3130 & 258.0000 & 1.0426 & $<0.0001$ \\
Year Smooth & 1.8607 & 19.0000 & 3.1724 & 0.0631 \\
Station Random Effect & 1.7293 & 7.0000 & 1.5247 & 0.1378 \\
\hline
\end{tabular}

Table A3.5: Best productivity model for the American Redstart (Setophaga ruticilla). The model was a quasi-Poisson GAMM with log link function. Based on AIC/QIC, support for including the noise level as a predictor of productivity was: strong; support for including the standard deviation of noise was: none. 


\begin{tabular}{lrrrr}
\hline A. parametric coefficients & Estimate & Std. Error & t-value & p-value \\
Intercept & -3.3482 & 1.8305 & -1.8291 & 0.0675 \\
Noise Level & -0.0957 & 0.0473 & -2.0216 & 0.0433 \\
Noise Standard Deviation & 0.5576 & 0.2594 & 2.1497 & 0.0317 \\
PC 1 & 0.1551 & 0.1573 & 0.9858 & 0.3243 \\
PC 2 & -0.2293 & 0.1199 & -1.9119 & 0.0560 \\
PC 3 & -0.6612 & 0.2301 & -2.8731 & 0.0041 \\
PC 4 & 0.1547 & 0.1257 & 1.2312 & 0.2184 \\
PC 5 & 0.1730 & 0.1550 & 1.1163 & 0.2644 \\
PC 6 & 0.4227 & 0.2245 & 1.8828 & 0.0599 \\
PC 7 & -0.2754 & 0.1356 & -2.0310 & 0.0424 \\
PC 8 & 0.2093 & 0.2234 & 0.9371 & 0.3488 \\
\hline B. smooth terms & edf & Ref.df & F-value & p-value \\
Location Smooth & 10.8871 & 19.0000 & 2557.0681 & 0.0058 \\
Year Smooth & 5.2537 & 7.0000 & 103.8002 & $<0.0001$ \\
Station Random Effect & 206.6827 & 272.0000 & 10.3051 & $<0.0001$ \\
\hline
\end{tabular}

Table A3.6: Best abundance model for the American Redstart (Setophaga ruticilla). The model was a quasi-Poisson GAMM with log link function. Based on AIC/QIC, support for including the noise level as a predictor of abundance was: weak; support for including the standard deviation of noise was: strong.

\begin{tabular}{lrrrr}
\hline A. parametric coefficients & Estimate & Std. Error & t-value & p-value \\
Intercept & -3.3731 & 0.7286 & -4.6295 & $<0.0001$ \\
Noise Level & 0.0522 & 0.0198 & 2.6306 & 0.0086 \\
PC 1 & 0.0379 & 0.0598 & 0.6334 & 0.5265 \\
PC 2 & 0.1401 & 0.0452 & 3.1016 & 0.0019 \\
PC 3 & 0.1095 & 0.0592 & 1.8503 & 0.0644 \\
PC 4 & -0.0127 & 0.0456 & -0.2794 & 0.7800 \\
PC 5 & -0.0606 & 0.0562 & -1.0773 & 0.2814 \\
PC 6 & -0.1283 & 0.0659 & -1.9467 & 0.0517 \\
PC 7 & 0.0903 & 0.0556 & 1.6227 & 0.1047 \\
PC 8 & -0.0481 & 0.0726 & -0.6617 & 0.5082 \\
\hline B. smooth terms & edf & Ref.df & F-value & p-value \\
Location Smooth & 307.6078 & 607.0000 & 2.6698 & $<0.0001$ \\
Year Smooth & 5.4690 & 19.0000 & 38.4965 & 0.0348 \\
Station Random Effect & 5.9820 & 7.0000 & 12.3412 & 0.0361 \\
\hline
\end{tabular}

Table A3.7: Best productivity model for the American Robin (Turdus migratorius). The model was a quasi-Poisson GAMM with log link function. Based on AIC/QIC, support for including the noise level as a predictor of productivity was: strong; support for including the standard deviation of noise was: none. 


\begin{tabular}{lrrrr}
\hline A. parametric coefficients & Estimate & Std. Error & t-value & p-value \\
Intercept & -5.7230 & 0.7946 & -7.2026 & $<0.0001$ \\
Noise Level & 0.0116 & 0.0214 & 0.5419 & 0.5879 \\
PC 1 & 0.2202 & 0.0614 & 3.5828 & 0.0003 \\
PC 2 & 0.0123 & 0.0441 & 0.2795 & 0.7799 \\
PC 3 & -0.1303 & 0.0568 & -2.2952 & 0.0218 \\
PC 4 & -0.0652 & 0.0447 & -1.4590 & 0.1446 \\
PC 5 & -0.0957 & 0.0548 & -1.7483 & 0.0805 \\
PC 6 & -0.0219 & 0.0604 & -0.3631 & 0.7166 \\
PC 7 & -0.0199 & 0.0550 & -0.3614 & 0.7178 \\
PC 8 & -0.0849 & 0.0679 & -1.2495 & 0.2115 \\
\hline B. smooth terms & edf & Ref.df & F-value & p-value \\
Location Smooth & 14.5356 & 19.0000 & 4729.4167 & $<0.0001$ \\
Year Smooth & 5.6922 & 7.0000 & 173.0054 & $<0.0001$ \\
Station Random Effect & 506.6956 & 637.0000 & 8.5346 & $<0.0001$ \\
\hline
\end{tabular}

Table A3.8: Best abundance model for the American Robin (Turdus migratorius). The model was a quasi-Poisson GAMM with log link function. Based on AIC/QIC, support for including the noise level as a predictor of abundance was: weak; support for including the standard deviation of noise was: none.

\begin{tabular}{lrrrr}
\hline A. parametric coefficients & Estimate & Std. Error & t-value & p-value \\
Intercept & -2.6963 & 0.3776 & -7.1402 & $<0.0001$ \\
PC 1 & 0.2097 & 0.1791 & 1.1706 & 0.2422 \\
PC 2 & 0.1713 & 0.1998 & 0.8576 & 0.3915 \\
PC 3 & 0.0040 & 0.1332 & 0.0300 & 0.9761 \\
PC 4 & 0.1157 & 0.1138 & 1.0166 & 0.3098 \\
PC 5 & 0.0110 & 0.1121 & 0.0984 & 0.9216 \\
PC 6 & -0.0816 & 0.1243 & -0.6560 & 0.5121 \\
PC 7 & 0.1390 & 0.1792 & 0.7754 & 0.4384 \\
PC 8 & 0.0549 & 0.2342 & 0.2345 & 0.8147 \\
\hline B. smooth terms & edf & Ref.df & F-value & p-value \\
Location Smooth & 55.3676 & 148.0000 & 0.8702 & $<0.0001$ \\
Year Smooth & 0.0004 & 19.0000 & 0.0000 & 0.7308 \\
Station Random Effect & 5.6358 & 7.0000 & 5.3877 & 0.0392 \\
\hline
\end{tabular}

Table A3.9: Best productivity model for the Ash-throated Flycatcher (Myiarchus cinerascens). The model was a quasi-Poisson GAMM with log link function. Based on AIC/QIC, support for including the noise level as a predictor of productivity was: none; support for including the standard deviation of noise was: none. 


\begin{tabular}{lrrrr}
\hline A. parametric coefficients & Estimate & Std. Error & t-value & p-value \\
Intercept & -6.0511 & 0.2915 & -20.7570 & $<0.0001$ \\
Noise Standard Deviation & -0.2026 & 0.1934 & -1.0479 & 0.2949 \\
PC 1 & -0.0118 & 0.1203 & -0.0977 & 0.9222 \\
PC 2 & 0.0609 & 0.1425 & 0.4274 & 0.6692 \\
PC 3 & 0.2494 & 0.0919 & 2.7154 & 0.0067 \\
PC 4 & -0.2344 & 0.0870 & -2.6949 & 0.0072 \\
PC 5 & -0.2046 & 0.0829 & -2.4669 & 0.0138 \\
PC 6 & -0.0044 & 0.0863 & -0.0510 & 0.9593 \\
PC 7 & -0.0768 & 0.1312 & -0.5855 & 0.5583 \\
PC 8 & 0.1848 & 0.1605 & 1.1513 & 0.2499 \\
\hline B. smooth terms & edf & Ref.df & F-value & p-value \\
Location Smooth & 11.8523 & 19.0000 & 74.0664 & 0.3836 \\
Year Smooth & 2.1086 & 7.0000 & 1.6268 & 0.3635 \\
Station Random Effect & 101.8911 & 149.0000 & 3.6268 & $<0.0001$ \\
\hline
\end{tabular}

Table A3.10: Best abundance model for the Ash-throated Flycatcher (Myiarchus cinerascens). The model was a quasi-Poisson GAMM with log link function. Based on AIC/QIC, support for including the noise level as a predictor of abundance was: none; support for including the standard deviation of noise was: strong.

\begin{tabular}{lrrrr}
\hline A. parametric coefficients & Estimate & Std. Error & t-value & p-value \\
Intercept & -1.3428 & 0.3130 & -4.2896 & $<0.0001$ \\
PC 1 & 0.2268 & 0.4113 & 0.5516 & 0.5813 \\
PC 2 & -0.0367 & 0.2851 & -0.1287 & 0.8976 \\
PC 3 & 0.0756 & 0.2027 & 0.3729 & 0.7093 \\
PC 4 & 0.2694 & 0.3561 & 0.7567 & 0.4494 \\
PC 5 & 0.3612 & 0.3212 & 1.1242 & 0.2611 \\
PC 6 & 0.1017 & 0.3067 & 0.3318 & 0.7401 \\
PC 7 & -0.0366 & 0.4325 & -0.0847 & 0.9325 \\
PC 8 & 0.9056 & 0.9846 & 0.9198 & 0.3579 \\
\hline B. smooth terms & edf & Ref.df & F-value & p-value \\
Location Smooth & 68.1433 & 194.0000 & 2.3328 & $<0.0001$ \\
Year Smooth & 5.3469 & 19.0000 & 14.1368 & 0.3694 \\
Station Random Effect & 4.8053 & 7.0000 & 13.1307 & $<0.0001$ \\
\hline
\end{tabular}

Table A3.11: Best productivity model for the Audubon's Warbler (Setophaga coronata auduboni). The model was a quasi-Poisson GAMM with log link function. Based on AIC/QIC, support for including the noise level as a predictor of productivity was: none; support for including the standard deviation of noise was: none. 


\begin{tabular}{lrrrr}
\hline A. parametric coefficients & Estimate & Std. Error & t-value & p-value \\
Intercept & -6.1749 & 0.2760 & -22.3736 & $<0.0001$ \\
Noise Standard Deviation & 0.4229 & 0.2122 & 1.9927 & 0.0464 \\
PC 1 & -0.5592 & 0.2361 & -2.3685 & 0.0180 \\
PC 2 & -0.1569 & 0.1680 & -0.9338 & 0.3505 \\
PC 3 & -0.1914 & 0.1223 & -1.5657 & 0.1176 \\
PC 4 & -0.0749 & 0.1790 & -0.4182 & 0.6759 \\
PC 5 & 0.0359 & 0.1686 & 0.2127 & 0.8316 \\
PC 6 & -0.0379 & 0.1834 & -0.2066 & 0.8364 \\
PC 7 & -0.2844 & 0.2367 & -1.2014 & 0.2297 \\
PC 8 & -0.0390 & 0.4898 & -0.0797 & 0.9365 \\
\hline B. smooth terms & edf & Ref.df & F-value & p-value \\
Location Smooth & 11.1191 & 19.0000 & 1767.6669 & 0.0016 \\
Year Smooth & 4.6043 & 7.0000 & 43.4057 & $<0.0001$ \\
Station Random Effect & 153.7531 & 194.0000 & 9.1552 & $<0.0001$ \\
\hline
\end{tabular}

Table A3.12: Best abundance model for the Audubon's Warbler (Setophaga coronata auduboni). The model was a quasi-Poisson GAMM with log link function. Based on AIC/QIC, support for including the noise level as a predictor of abundance was: none; support for including the standard deviation of noise was: weak.

\begin{tabular}{lrrrr}
\hline A. parametric coefficients & Estimate & Std. Error & t-value & p-value \\
Intercept & -0.1873 & 0.1012 & -1.8505 & 0.0644 \\
Noise Standard Deviation & -0.1769 & 0.0955 & -1.8513 & 0.0643 \\
PC 1 & 0.0603 & 0.0326 & 1.8484 & 0.0647 \\
PC 2 & 0.0271 & 0.0412 & 0.6575 & 0.5109 \\
PC 3 & -0.0277 & 0.0577 & -0.4809 & 0.6306 \\
PC 4 & -0.0650 & 0.0420 & -1.5469 & 0.1221 \\
PC 5 & 0.0127 & 0.0532 & 0.2380 & 0.8119 \\
PC 6 & 0.0837 & 0.0574 & 1.4574 & 0.1452 \\
PC 7 & 0.0056 & 0.0478 & 0.1162 & 0.9075 \\
PC 8 & -0.0227 & 0.0593 & -0.3826 & 0.7021 \\
\hline B. smooth terms & edf & Ref.df & F-value & p-value \\
Location Smooth & 134.6444 & 331.0000 & 1.4308 & $<0.0001$ \\
Year Smooth & 10.0831 & 19.0000 & 29.4261 & $<0.0001$ \\
Station Random Effect & 0.0014 & 7.0000 & 0.0001 & 0.4870 \\
\hline
\end{tabular}

Table A3.13: Best productivity model for the Black-capped Chickadee (Poecile atricapillus). The model was a quasi-Poisson GAMM with log link function. Based on AIC/QIC, support for including the noise level as a predictor of productivity was: none; support for including the standard deviation of noise was: strong. 


\begin{tabular}{lrrrr}
\hline A. parametric coefficients & Estimate & Std. Error & t-value & p-value \\
Intercept & -7.0465 & 0.9919 & -7.1038 & $<0.0001$ \\
Noise Level & 0.0459 & 0.0265 & 1.7322 & 0.0834 \\
PC 1 & 0.1359 & 0.0690 & 1.9702 & 0.0489 \\
PC 2 & 0.1119 & 0.0572 & 1.9558 & 0.0506 \\
PC 3 & 0.0096 & 0.0848 & 0.1127 & 0.9103 \\
PC 4 & -0.0772 & 0.0541 & -1.4276 & 0.1535 \\
PC 5 & -0.0352 & 0.0683 & -0.5153 & 0.6064 \\
PC 6 & -0.1204 & 0.0831 & -1.4481 & 0.1477 \\
PC 7 & -0.0249 & 0.0648 & -0.3835 & 0.7014 \\
PC 8 & -0.0127 & 0.0788 & -0.1608 & 0.8722 \\
\hline B. smooth terms & edf & Ref.df & F-value & p-value \\
Location Smooth & 14.9680 & 19.0000 & 1042.2007 & $<0.0001$ \\
Year Smooth & 3.9038 & 7.0000 & 82.2930 & $<0.0001$ \\
Station Random Effect & 263.4399 & 347.0000 & 5.5480 & $<0.0001$ \\
\hline
\end{tabular}

Table A3.14: Best abundance model for the Black-capped Chickadee (Poecile atricapillus). The model was a quasi-Poisson GAMM with log link function. Based on AIC/QIC, support for including the noise level as a predictor of abundance was: weak; support for including the standard deviation of noise was: none.

\begin{tabular}{lrrrr}
\hline A. parametric coefficients & Estimate & Std. Error & t-value & p-value \\
Intercept & -2.0586 & 0.2002 & -10.2847 & $<0.0001$ \\
PC 1 & 0.1545 & 0.1100 & 1.4040 & 0.1610 \\
PC 2 & -0.0253 & 0.1437 & -0.1761 & 0.8603 \\
PC 3 & 0.2652 & 0.1041 & 2.5482 & 0.0112 \\
PC 4 & 0.1897 & 0.1373 & 1.3816 & 0.1678 \\
PC 5 & 0.2162 & 0.1295 & 1.6702 & 0.0956 \\
PC 6 & -0.0167 & 0.1118 & -0.1495 & 0.8813 \\
PC 7 & 0.0870 & 0.1916 & 0.4541 & 0.6500 \\
PC 8 & 0.0482 & 0.1754 & 0.2750 & 0.7834 \\
\hline B. smooth terms & edf & Ref.df & F-value & p-value \\
Location Smooth & 22.5160 & 128.0000 & 0.2599 & 0.0055 \\
Year Smooth & 5.1232 & 19.0000 & 2.5231 & 0.0019 \\
Station Random Effect & 5.4445 & 7.0000 & 5.2407 & 0.0003 \\
\hline
\end{tabular}

Table A3.15: Best productivity model for the Black-chinned Hummingbird (Archilochus alexandri). The model was a quasi-Poisson GAMM with log link function. Based on AIC/QIC, support for including the noise level as a predictor of productivity was: none; support for including the standard deviation of noise was: none. 


\begin{tabular}{lrrrr}
\hline A. parametric coefficients & Estimate & Std. Error & t-value & p-value \\
Intercept & -7.9847 & 1.4577 & -5.4776 & $<0.0001$ \\
Noise Level & 0.0304 & 0.0397 & 0.7672 & 0.4431 \\
PC 1 & 0.2863 & 0.1425 & 2.0089 & 0.0448 \\
PC 2 & 0.2334 & 0.1387 & 1.6829 & 0.0927 \\
PC 3 & 0.1414 & 0.0996 & 1.4192 & 0.1561 \\
PC 4 & -0.1509 & 0.1103 & -1.3677 & 0.1717 \\
PC 5 & -0.0919 & 0.1092 & -0.8417 & 0.4001 \\
PC 6 & -0.0481 & 0.1066 & -0.4508 & 0.6522 \\
PC 7 & 0.1518 & 0.1583 & 0.9591 & 0.3377 \\
PC 8 & -0.0054 & 0.1736 & -0.0308 & 0.9754 \\
\hline B. smooth terms & edf & Ref.df & F-value & p-value \\
Location Smooth & 10.8660 & 19.0000 & 112.8999 & 0.0027 \\
Year Smooth & 4.6568 & 7.0000 & 17.6253 & $<0.0001$ \\
Station Random Effect & 88.4969 & 149.0000 & 2.7157 & $<0.0001$ \\
\hline
\end{tabular}

Table A3.16: Best abundance model for the Black-chinned Hummingbird (Archilochus alexandri). The model was a quasi-Poisson GAMM with log link function. Based on AIC/QIC, support for including the noise level as a predictor of abundance was: weak; support for including the standard deviation of noise was: none.

\begin{tabular}{lrrrr}
\hline A. parametric coefficients & Estimate & Std. Error & t-value & p-value \\
Intercept & 0.0423 & 0.0894 & 0.4730 & 0.6363 \\
PC 1 & 0.0561 & 0.0451 & 1.2436 & 0.2139 \\
PC 2 & 0.0448 & 0.0516 & 0.8679 & 0.3856 \\
PC 3 & -0.0146 & 0.0411 & -0.3546 & 0.7229 \\
PC 4 & 0.0441 & 0.0350 & 1.2601 & 0.2079 \\
PC 5 & 0.0546 & 0.0373 & 1.4617 & 0.1441 \\
PC 6 & 0.0223 & 0.0360 & 0.6182 & 0.5366 \\
PC 7 & -0.0719 & 0.0438 & -1.6422 & 0.1008 \\
PC 8 & -0.0918 & 0.0578 & -1.5899 & 0.1121 \\
\hline B. smooth terms & edf & Ref.df & F-value & p-value \\
Location Smooth & 75.8113 & 222.0000 & 0.8799 & $<0.0001$ \\
Year Smooth & 8.3592 & 19.0000 & 10.7069 & 0.0001 \\
Station Random Effect & 0.0011 & 7.0000 & 0.0000 & 0.7215 \\
\hline
\end{tabular}

Table A3.17: Best productivity model for the Bewick's Wren (Thryomanes bewickii). The model was a quasi-Poisson GAMM with log link function. Based on AIC/QIC, support for including the noise level as a predictor of productivity was: none; support for including the standard deviation of noise was: none. 


\begin{tabular}{lrrrr}
\hline A. parametric coefficients & Estimate & Std. Error & t-value & p-value \\
Intercept & -7.2381 & 1.1159 & -6.4866 & $<0.0001$ \\
Noise Level & 0.0359 & 0.0301 & 1.1924 & 0.2333 \\
PC 1 & 0.4829 & 0.0991 & 4.8717 & $<0.0001$ \\
PC 2 & 0.5611 & 0.0878 & 6.3939 & $<0.0001$ \\
PC 3 & 0.2051 & 0.0771 & 2.6583 & 0.0079 \\
PC 4 & -0.1392 & 0.0735 & -1.8942 & 0.0584 \\
PC 5 & 0.0286 & 0.0767 & 0.3729 & 0.7093 \\
PC 6 & -0.0979 & 0.0751 & -1.3047 & 0.1922 \\
PC 7 & 0.0060 & 0.0996 & 0.0604 & 0.9518 \\
PC 8 & -0.2243 & 0.0961 & -2.3336 & 0.0197 \\
\hline B. smooth terms & edf & Ref.df & F-value & p-value \\
Location Smooth & 13.6171 & 19.0000 & 1291.7416 & 0.0064 \\
Year Smooth & 6.3564 & 7.0000 & 50.2081 & 0.0035 \\
Station Random Effect & 190.2886 & 246.0000 & 6.3088 & $<0.0001$ \\
\hline
\end{tabular}

Table A3.18: Best abundance model for the Bewick's Wren (Thryomanes bewickii). The model was a quasi-Poisson GAMM with log link function. Based on AIC/QIC, support for including the noise level as a predictor of abundance was: weak; support for including the standard deviation of noise was: none.

\begin{tabular}{lrrrr}
\hline A. parametric coefficients & Estimate & Std. Error & t-value & p-value \\
Intercept & -3.5978 & 1.2746 & -2.8225 & 0.0048 \\
Noise Level & 0.0402 & 0.0365 & 1.1031 & 0.2701 \\
Noise Standard Deviation & 0.2941 & 0.1724 & 1.7056 & 0.0883 \\
PC 1 & 0.0092 & 0.1225 & 0.0749 & 0.9403 \\
PC 2 & -0.1386 & 0.0977 & -1.4193 & 0.1560 \\
PC 3 & 0.1209 & 0.0854 & 1.4161 & 0.1569 \\
PC 4 & -0.0382 & 0.1013 & -0.3772 & 0.7061 \\
PC 5 & 0.0696 & 0.1125 & 0.6189 & 0.5361 \\
PC 6 & -0.0656 & 0.1016 & -0.6455 & 0.5187 \\
PC 7 & 0.0146 & 0.1505 & 0.0972 & 0.9226 \\
PC 8 & 0.0552 & 0.2425 & 0.2278 & 0.8199 \\
\hline B. smooth terms & edf & Ref.df & F-value & p-value \\
Location Smooth & 147.4505 & 336.0000 & 1.8884 & $<0.0001$ \\
Year Smooth & 8.7913 & 19.0000 & 126.5078 & 0.0001 \\
Station Random Effect & 4.3344 & 7.0000 & 9.6514 & 0.0044 \\
\hline
\end{tabular}

Table A3.19: Best productivity model for the Black-headed Grosbeak (Pheucticus melanocephalus). The model was a quasi-Poisson GAMM with log link function. Based on AIC/QIC, support for including the noise level as a predictor of productivity was: weak; support for including the standard deviation of noise was: weak. 


\begin{tabular}{lrrrr}
\hline A. parametric coefficients & Estimate & Std. Error & t-value & p-value \\
Intercept & -5.6345 & 0.1587 & -35.5058 & $<0.0001$ \\
Noise Standard Deviation & 0.2087 & 0.1492 & 1.3990 & 0.1619 \\
PC 1 & -0.0236 & 0.0786 & -0.3004 & 0.7639 \\
PC 2 & 0.2273 & 0.0807 & 2.8155 & 0.0049 \\
PC 3 & 0.1081 & 0.0697 & 1.5498 & 0.1213 \\
PC 4 & -0.2049 & 0.0787 & -2.6021 & 0.0093 \\
PC 5 & -0.0296 & 0.0858 & -0.3443 & 0.7306 \\
PC 6 & -0.0164 & 0.0811 & -0.2020 & 0.8399 \\
PC 7 & -0.0212 & 0.1187 & -0.1786 & 0.8583 \\
PC 8 & -0.2684 & 0.1986 & -1.3511 & 0.1768 \\
\hline B. smooth terms & edf & Ref.df & F-value & p-value \\
Location Smooth & 5.6946 & 19.0000 & 501.6604 & 0.0354 \\
Year Smooth & 4.9862 & 7.0000 & 49.4116 & 0.0001 \\
Station Random Effect & 284.4565 & 339.0000 & 9.7032 & $<0.0001$ \\
\hline
\end{tabular}

Table A3.20: Best abundance model for the Black-headed Grosbeak (Pheucticus melanocephalus). The model was a quasi-Poisson GAMM with log link function. Based on AIC/QIC, support for including the noise level as a predictor of abundance was: none; support for including the standard deviation of noise was: weak. 


\begin{tabular}{lrrrr}
\hline A. parametric coefficients & Estimate & Std. Error & t-value & p-value \\
Intercept & 241.9885 & 849.1624 & 0.2850 & 0.7767 \\
PC 1 & 5.8962 & 78.0746 & 0.0755 & 0.9401 \\
PC 2 & 344.0676 & 731.9009 & 0.4701 & 0.6400 \\
PC 3 & 760.4124 & 1812.7817 & 0.4195 & 0.6764 \\
PC 4 & 85.5022 & 247.2369 & 0.3458 & 0.7307 \\
PC 5 & -172.8094 & 414.9852 & -0.4164 & 0.6786 \\
PC 6 & -493.8407 & 1187.9236 & -0.4157 & 0.6792 \\
PC 7 & 224.7659 & 536.4689 & 0.4190 & 0.6768 \\
PC 8 & -83.1538 & 179.9530 & -0.4621 & 0.6458 \\
\hline B. smooth terms & edf & Ref.df & F-value & p-value \\
Location Smooth & 2.6753 & 11.0000 & 0.5260 & 0.0573 \\
Year Smooth & 0.0000 & 12.0000 & 0.0000 & 1.0000 \\
Station Random Effect & 2.4670 & 7.0000 & 1.5394 & 0.0150 \\
\hline
\end{tabular}

Table A3.21: Best productivity model for the Bobolink (Dolichonyx oryzivorus). The model was a quasi-Poisson GAMM with log link function. Based on AIC/QIC, support for including the noise level as a predictor of productivity was: none; support for including the standard deviation of noise was: none. 


\begin{tabular}{lrrrr}
\hline A. parametric coefficients & Estimate & Std. Error & t-value & p-value \\
Intercept & -61.6408 & 17.3759 & -3.5475 & 0.0006 \\
Noise Level & 0.3970 & 0.1865 & 2.1292 & 0.0356 \\
PC 1 & 5.3710 & 3.4004 & 1.5795 & 0.1173 \\
PC 2 & -9.9407 & 4.4909 & -2.2135 & 0.0291 \\
PC 3 & -45.2715 & 17.1151 & -2.6451 & 0.0094 \\
PC 4 & -8.3728 & 2.7242 & -3.0736 & 0.0027 \\
PC 5 & 9.7500 & 3.4113 & 2.8581 & 0.0052 \\
PC 6 & 31.3942 & 11.9804 & 2.6205 & 0.0101 \\
PC 7 & -14.0158 & 5.6204 & -2.4937 & 0.0142 \\
PC 8 & -0.6568 & 2.4184 & -0.2716 & 0.7865 \\
\hline B. smooth terms & edf & Ref.df & F-value & p-value \\
Location Smooth & 0.0000 & 15.0000 & 0.0000 & 0.1009 \\
Year Smooth & 1.3381 & 7.0000 & 1.6193 & 0.0006 \\
Station Random Effect & 0.0124 & 13.0000 & 0.0014 & 0.1532 \\
\hline
\end{tabular}

Table A3.22: Best abundance model for the Bobolink (Dolichonyx oryzivorus). The model was a quasi-Poisson GAMM with log link function. Based on AIC/QIC, support for including the noise level as a predictor of abundance was: weak; support for including the standard deviation of noise was: none.

\begin{tabular}{lrrrr}
\hline A. parametric coefficients & Estimate & Std. Error & t-value & p-value \\
Intercept & -0.1415 & 0.0832 & -1.7000 & 0.0896 \\
PC 1 & 0.0873 & 0.0407 & 2.1454 & 0.0322 \\
PC 2 & 0.0783 & 0.0504 & 1.5531 & 0.1208 \\
PC 3 & -0.0572 & 0.0469 & -1.2189 & 0.2233 \\
PC 4 & -0.0488 & 0.0395 & -1.2353 & 0.2171 \\
PC 5 & 0.1060 & 0.0424 & 2.5017 & 0.0126 \\
PC 6 & 0.0787 & 0.0449 & 1.7538 & 0.0799 \\
PC 7 & 0.0210 & 0.0512 & 0.4099 & 0.6820 \\
PC 8 & 0.0278 & 0.0525 & 0.5301 & 0.5962 \\
\hline B. smooth terms & edf & Ref.df & F-value & p-value \\
Location Smooth & 37.7975 & 191.0000 & 0.3895 & $<0.0001$ \\
Year Smooth & 5.2956 & 19.0000 & 8.3461 & $<0.0001$ \\
Station Random Effect & 4.5758 & 7.0000 & 4.6005 & 0.0131 \\
\hline
\end{tabular}

Table A3.23: Best productivity model for the Bushtit (Psaltriparus minimus). The model was a quasi-Poisson GAMM with log link function. Based on AIC/QIC, support for including the noise level as a predictor of productivity was: none; support for including the standard deviation of noise was: none. 


\begin{tabular}{lrrrr}
\hline A. parametric coefficients & Estimate & Std. Error & t-value & p-value \\
Intercept & -8.3768 & 1.1303 & -7.4112 & $<0.0001$ \\
Noise Level & 0.0684 & 0.0312 & 2.1926 & 0.0285 \\
Noise Standard Deviation & -0.1036 & 0.1795 & -0.5771 & 0.5640 \\
PC 1 & 0.2336 & 0.0989 & 2.3619 & 0.0183 \\
PC 2 & 0.2296 & 0.0892 & 2.5735 & 0.0102 \\
PC 3 & 0.1533 & 0.0858 & 1.7868 & 0.0742 \\
PC 4 & -0.0796 & 0.0813 & -0.9792 & 0.3276 \\
PC 5 & -0.0371 & 0.0870 & -0.4261 & 0.6701 \\
PC 6 & -0.1292 & 0.0919 & -1.4053 & 0.1601 \\
PC 7 & -0.2308 & 0.1139 & -2.0263 & 0.0429 \\
PC 8 & -0.2197 & 0.1025 & -2.1446 & 0.0321 \\
\hline B. smooth terms & edf & Ref.df & F-value & p-value \\
Location Smooth & 9.3771 & 19.0000 & 78.1211 & 0.0150 \\
Year Smooth & 2.9076 & 7.0000 & 23.0472 & 0.0002 \\
Station Random Effect & 136.9324 & 205.0000 & 3.3576 & $<0.0001$ \\
\hline
\end{tabular}

Table A3.24: Best abundance model for the Bushtit (Psaltriparus minimus). The model was a quasi-Poisson GAMM with log link function. Based on AIC/QIC, support for including the noise level as a predictor of abundance was: weak; support for including the standard deviation of noise was: weak.

\begin{tabular}{lrrrr}
\hline A. parametric coefficients & Estimate & Std. Error & t-value & p-value \\
Intercept & -3.4361 & 1.2832 & -2.6777 & 0.0077 \\
Noise Standard Deviation & -0.2245 & 0.4662 & -0.4816 & 0.6303 \\
PC 1 & -0.4102 & 0.5143 & -0.7975 & 0.4256 \\
PC 2 & -1.2898 & 0.8106 & -1.5912 & 0.1123 \\
PC 3 & 0.3199 & 0.6629 & 0.4825 & 0.6297 \\
PC 4 & -2.8095 & 2.0674 & -1.3589 & 0.1749 \\
PC 5 & 3.5520 & 1.9252 & 1.8451 & 0.0657 \\
PC 6 & -2.0730 & 1.0577 & -1.9599 & 0.0507 \\
PC 7 & 0.5724 & 1.7781 & 0.3219 & 0.7477 \\
PC 8 & -0.7182 & 1.3832 & -0.5192 & 0.6039 \\
\hline B. smooth terms & edf & Ref.df & F-value & p-value \\
Location Smooth & 26.5768 & 80.0000 & 0.5908 & 0.0020 \\
Year Smooth & 0.0004 & 19.0000 & 0.0000 & 0.9825 \\
Station Random Effect & 4.5409 & 7.0000 & 4.2274 & 0.0158 \\
\hline
\end{tabular}

Table A3.25: Best productivity model for the Cassin's Finch (Haemorhous cassinii). The model was a quasi-Poisson GAMM with log link function. Based on AIC/QIC, support for including the noise level as a predictor of productivity was: none; support for including the standard deviation of noise was: weak. 


\begin{tabular}{lrrrr}
\hline A. parametric coefficients & Estimate & Std. Error & t-value & p-value \\
Intercept & -6.5465 & 0.3422 & -19.1321 & $<0.0001$ \\
PC 1 & 0.3351 & 0.3712 & 0.9029 & 0.3668 \\
PC 2 & -0.4436 & 0.3098 & -1.4322 & 0.1524 \\
PC 3 & 0.0436 & 0.2310 & 0.1887 & 0.8504 \\
PC 4 & -0.0458 & 0.5648 & -0.0811 & 0.9354 \\
PC 5 & 0.7401 & 0.5583 & 1.3257 & 0.1852 \\
PC 6 & -0.5035 & 0.3503 & -1.4374 & 0.1509 \\
PC 7 & 0.2221 & 0.5686 & 0.3906 & 0.6962 \\
PC 8 & 0.0186 & 0.9722 & 0.0192 & 0.9847 \\
\hline B. smooth terms & edf & Ref.df & F-value & p-value \\
Location Smooth & 8.8941 & 19.0000 & 119.6817 & 0.0174 \\
Year Smooth & 3.9498 & 7.0000 & 3.5014 & 0.0035 \\
Station Random Effect & 56.9732 & 85.0000 & 3.5740 & $<0.0001$ \\
\hline
\end{tabular}

Table A3.26: Best abundance model for the Cassin's Finch (Haemorhous cassinii). The model was a quasi-Poisson GAMM with log link function. Based on AIC/QIC, support for including the noise level as a predictor of abundance was: none; support for including the standard deviation of noise was: none.

\begin{tabular}{lrrrr}
\hline A. parametric coefficients & Estimate & Std. Error & t-value & p-value \\
Intercept & -1.5683 & 0.7437 & -2.1088 & 0.0351 \\
Noise Level & 0.0286 & 0.0184 & 1.5566 & 0.1197 \\
Noise Standard Deviation & -0.1584 & 0.1130 & -1.4025 & 0.1609 \\
PC 1 & -0.1938 & 0.0690 & -2.8063 & 0.0051 \\
PC 2 & 0.0139 & 0.0563 & 0.2473 & 0.8047 \\
PC 3 & -0.0697 & 0.0882 & -0.7905 & 0.4293 \\
PC 4 & -0.0126 & 0.0642 & -0.1957 & 0.8449 \\
PC 5 & -0.0067 & 0.0732 & -0.0915 & 0.9271 \\
PC 6 & 0.0965 & 0.0782 & 1.2348 & 0.2170 \\
PC 7 & -0.0973 & 0.0654 & -1.4867 & 0.1373 \\
PC 8 & -0.2501 & 0.1030 & -2.4289 & 0.0152 \\
\hline B. smooth terms & edf & Ref.df & F-value & p-value \\
Location Smooth & 143.4443 & 332.0000 & 1.3249 & $<0.0001$ \\
Year Smooth & 5.5145 & 19.0000 & 10.3885 & 0.0025 \\
Station Random Effect & 1.6470 & 7.0000 & 3.2895 & 0.0063 \\
\hline
\end{tabular}

Table A3.27: Best productivity model for the Carolina Wren (Thryothorus ludovicianus). The model was a quasi-Poisson GAMM with log link function. Based on AIC/QIC, support for including the noise level as a predictor of productivity was: weak; support for including the standard deviation of noise was: weak. 


\begin{tabular}{lrrrr}
\hline A. parametric coefficients & Estimate & Std. Error & t-value & p-value \\
Intercept & -8.5026 & 0.9000 & -9.4472 & $<0.0001$ \\
Noise Level & 0.0759 & 0.0225 & 3.3729 & 0.0008 \\
PC 1 & 0.1038 & 0.0711 & 1.4595 & 0.1445 \\
PC 2 & 0.1131 & 0.0638 & 1.7725 & 0.0764 \\
PC 3 & -0.3183 & 0.1162 & -2.7392 & 0.0062 \\
PC 4 & 0.0567 & 0.0807 & 0.7021 & 0.4827 \\
PC 5 & -0.0184 & 0.0918 & -0.2007 & 0.8410 \\
PC 6 & 0.1792 & 0.0943 & 1.9001 & 0.0575 \\
PC 7 & -0.0515 & 0.0828 & -0.6225 & 0.5336 \\
PC 8 & -0.1126 & 0.0824 & -1.3665 & 0.1719 \\
\hline B. smooth terms & edf & Ref.df & F-value & p-value \\
Location Smooth & 12.2227 & 19.0000 & 1506.1239 & $<0.0001$ \\
Year Smooth & 5.6918 & 7.0000 & 78.4667 & $<0.0001$ \\
Station Random Effect & 264.6517 & 366.0000 & 3.9928 & $<0.0001$ \\
\hline
\end{tabular}

Table A3.28: Best abundance model for the Carolina Wren (Thryothorus ludovicianus). The model was a quasi-Poisson GAMM with log link function. Based on AIC/QIC, support for including the noise level as a predictor of abundance was: weak; support for including the standard deviation of noise was: none.

\begin{tabular}{lrrrr}
\hline A. parametric coefficients & Estimate & Std. Error & t-value & p-value \\
Intercept & 2.8389 & 5.7203 & 0.4963 & 0.6199 \\
Noise Level & -0.2942 & 0.1374 & -2.1417 & 0.0327 \\
Noise Standard Deviation & 0.8211 & 0.3571 & 2.2995 & 0.0219 \\
PC 1 & -7.2553 & 5.6719 & -1.2792 & 0.2014 \\
PC 2 & 3.5619 & 3.2105 & 1.1095 & 0.2677 \\
PC 3 & -1.1850 & 0.8750 & -1.3543 & 0.1762 \\
PC 4 & -1.7115 & 1.3297 & -1.2872 & 0.1986 \\
PC 5 & 0.9505 & 0.6820 & 1.3937 & 0.1640 \\
PC 6 & 1.3344 & 1.3052 & 1.0224 & 0.3071 \\
PC 7 & 1.0495 & 0.8561 & 1.2259 & 0.2208 \\
PC 8 & -11.5740 & 7.4724 & -1.5489 & 0.1220 \\
\hline B. smooth terms & edf & Ref.df & F-value & p-value \\
Location Smooth & 41.7476 & 129.0000 & 0.9929 & $<0.0001$ \\
Year Smooth & 6.3003 & 19.0000 & 15.8998 & 0.1600 \\
Station Random Effect & 1.6528 & 7.0000 & 0.5214 & 0.2043 \\
\hline
\end{tabular}

Table A3.29: Best productivity model for the Cassin's Vireo (Vireo cassinii). The model was a quasi-Poisson GAMM with log link function. Based on AIC/QIC, support for including the noise level as a predictor of productivity was: strong; support for including the standard deviation of noise was: strong. 


\begin{tabular}{lrrrr}
\hline A. parametric coefficients & Estimate & Std. Error & t-value & p-value \\
Intercept & -4.5487 & 2.9299 & -1.5525 & 0.1208 \\
Noise Level & -0.0706 & 0.0829 & -0.8514 & 0.3947 \\
PC 1 & -0.1157 & 0.3035 & -0.3811 & 0.7032 \\
PC 2 & -0.0338 & 0.1952 & -0.1730 & 0.8627 \\
PC 3 & 0.1723 & 0.1649 & 1.0450 & 0.2962 \\
PC 4 & -0.1234 & 0.1968 & -0.6270 & 0.5308 \\
PC 5 & -0.0202 & 0.1998 & -0.1012 & 0.9194 \\
PC 6 & -0.1801 & 0.1815 & -0.9919 & 0.3214 \\
PC 7 & -0.4093 & 0.2960 & -1.3827 & 0.1670 \\
PC 8 & -0.4566 & 0.5082 & -0.8984 & 0.3691 \\
\hline B. smooth terms & edf & Ref.df & F-value & p-value \\
Location Smooth & 4.3253 & 19.0000 & 75.2618 & 0.3160 \\
Year Smooth & 4.0510 & 7.0000 & 10.2045 & 0.0001 \\
Station Random Effect & 116.4088 & 146.0000 & 8.2133 & $<0.0001$ \\
\hline
\end{tabular}

Table A3.30: Best abundance model for the Cassin's Vireo (Vireo cassinii). The model was a quasi-Poisson GAMM with log link function. Based on AIC/QIC, support for including the noise level as a predictor of abundance was: strong; support for including the standard deviation of noise was: none.

\begin{tabular}{lrrrr}
\hline A. parametric coefficients & Estimate & Std. Error & t-value & p-value \\
Intercept & -6.2426 & 3.5839 & -1.7419 & 0.0818 \\
Noise Level & 0.0548 & 0.0945 & 0.5805 & 0.5617 \\
Noise Standard Deviation & 0.1575 & 0.5742 & 0.2742 & 0.7840 \\
PC 1 & -0.2935 & 0.3832 & -0.7661 & 0.4438 \\
PC 2 & 0.1534 & 0.2735 & 0.5608 & 0.5751 \\
PC 3 & 0.1695 & 0.2980 & 0.5689 & 0.5696 \\
PC 4 & -0.0470 & 0.2094 & -0.2244 & 0.8225 \\
PC 5 & -0.0233 & 0.2797 & -0.0834 & 0.9335 \\
PC 6 & 0.2029 & 0.3428 & 0.5920 & 0.5540 \\
PC 7 & 0.0556 & 0.2467 & 0.2254 & 0.8217 \\
PC 8 & -0.0624 & 0.6243 & -0.0999 & 0.9204 \\
\hline B. smooth terms & edf & Ref.df & F-value & p-value \\
Location Smooth & 108.8276 & 284.0000 & 0.7956 & $<0.0001$ \\
Year Smooth & 0.0023 & 19.0000 & 0.0001 & 0.6026 \\
Station Random Effect & 4.3043 & 7.0000 & 18.0158 & 0.0956 \\
\hline
\end{tabular}

Table A3.31: Best productivity model for the Cedar Waxwing (Bombycilla cedrorum). The model was a quasi-Poisson GAMM with log link function. Based on AIC/QIC, support for including the noise level as a predictor of productivity was: strong; support for including the standard deviation of noise was: strong. 


\begin{tabular}{lrrrr}
\hline A. parametric coefficients & Estimate & Std. Error & t-value & p-value \\
Intercept & -6.8835 & 1.5092 & -4.5611 & $<0.0001$ \\
Noise Level & 0.0238 & 0.0403 & 0.5897 & 0.5555 \\
Noise Standard Deviation & 0.0436 & 0.2136 & 0.2040 & 0.8384 \\
PC 1 & 0.0630 & 0.1181 & 0.5334 & 0.5938 \\
PC 2 & 0.2728 & 0.0891 & 3.0608 & 0.0022 \\
PC 3 & 0.0504 & 0.1270 & 0.3968 & 0.6915 \\
PC 4 & -0.0523 & 0.0798 & -0.6554 & 0.5122 \\
PC 5 & 0.0511 & 0.1046 & 0.4888 & 0.6250 \\
PC 6 & 0.1410 & 0.1343 & 1.0505 & 0.2936 \\
PC 7 & -0.0541 & 0.0955 & -0.5664 & 0.5712 \\
PC 8 & 0.1476 & 0.1469 & 1.0048 & 0.3151 \\
\hline B. smooth terms & edf & Ref.df & F-value & p-value \\
Location Smooth & 10.8692 & 19.0000 & 1070.3742 & 0.0004 \\
Year Smooth & 3.9320 & 7.0000 & 99.4260 & $<0.0001$ \\
Station Random Effect & 210.4918 & 284.0000 & 5.6006 & $<0.0001$ \\
\hline
\end{tabular}

Table A3.32: Best abundance model for the Cedar Waxwing (Bombycilla cedrorum). The model was a quasi-Poisson GAMM with log link function. Based on AIC/QIC, support for including the noise level as a predictor of abundance was: weak; support for including the standard deviation of noise was: weak.

\begin{tabular}{lrrrr}
\hline A. parametric coefficients & Estimate & Std. Error & t-value & p-value \\
Intercept & -1.8152 & 0.2633 & -6.8932 & $<0.0001$ \\
Noise Standard Deviation & 0.1101 & 0.2096 & 0.5254 & 0.5994 \\
PC 1 & -0.0261 & 0.2181 & -0.1199 & 0.9046 \\
PC 2 & -0.0327 & 0.1599 & -0.2044 & 0.8381 \\
PC 3 & 0.1414 & 0.1017 & 1.3897 & 0.1649 \\
PC 4 & -0.2262 & 0.1461 & -1.5488 & 0.1217 \\
PC 5 & 0.0685 & 0.1453 & 0.4714 & 0.6374 \\
PC 6 & 0.0753 & 0.1499 & 0.5024 & 0.6155 \\
PC 7 & 0.0026 & 0.1784 & 0.0144 & 0.9885 \\
PC 8 & -0.2438 & 0.3749 & -0.6503 & 0.5156 \\
\hline B. smooth terms & edf & Ref.df & F-value & p-value \\
Location Smooth & 100.3499 & 294.0000 & 0.8624 & $<0.0001$ \\
Year Smooth & 0.0004 & 19.0000 & 0.0000 & 1.0000 \\
Station Random Effect & 4.3460 & 7.0000 & 3.9805 & 0.2584 \\
\hline
\end{tabular}

Table A3.33: Best productivity model for the Chipping Sparrow (Spizella passerina). The model was a quasi-Poisson GAMM with log link function. Based on AIC/QIC, support for including the noise level as a predictor of productivity was: none; support for including the standard deviation of noise was: weak. 


\begin{tabular}{lrrrr}
\hline A. parametric coefficients & Estimate & Std. Error & t-value & p-value \\
Intercept & -4.7767 & 1.5386 & -3.1046 & 0.0019 \\
Noise Level & -0.0579 & 0.0429 & -1.3494 & 0.1773 \\
Noise Standard Deviation & 0.3523 & 0.2037 & 1.7300 & 0.0837 \\
PC 1 & -0.2199 & 0.1844 & -1.1927 & 0.2331 \\
PC 2 & -0.0508 & 0.1343 & -0.3781 & 0.7054 \\
PC 3 & 0.1205 & 0.1154 & 1.0443 & 0.2964 \\
PC 4 & -0.0663 & 0.1164 & -0.5701 & 0.5687 \\
PC 5 & 0.0493 & 0.1218 & 0.4050 & 0.6855 \\
PC 6 & 0.2667 & 0.1381 & 1.9313 & 0.0535 \\
PC 7 & 0.1031 & 0.1516 & 0.6798 & 0.4967 \\
PC 8 & 0.0634 & 0.3245 & 0.1954 & 0.8451 \\
\hline B. smooth terms & edf & Ref.df & F-value & p-value \\
Location Smooth & 3.3000 & 19.0000 & 115.5005 & 0.1835 \\
Year Smooth & 4.8780 & 7.0000 & 74.0300 & $<0.0001$ \\
Station Random Effect & 245.6036 & 313.0000 & 8.5210 & $<0.0001$ \\
\hline
\end{tabular}

Table A3.34: Best abundance model for the Chipping Sparrow (Spizella passerina). The model was a quasi-Poisson GAMM with log link function. Based on AIC/QIC, support for including the noise level as a predictor of abundance was: weak; support for including the standard deviation of noise was: weak.

\begin{tabular}{lrrrr}
\hline A. parametric coefficients & Estimate & Std. Error & t-value & p-value \\
Intercept & -1.5023 & 0.0509 & -29.4905 & $<0.0001$ \\
PC 1 & 0.0427 & 0.0407 & 1.0482 & 0.2946 \\
PC 2 & 0.1201 & 0.0435 & 2.7610 & 0.0058 \\
PC 3 & -0.0597 & 0.0625 & -0.9556 & 0.3394 \\
PC 4 & -0.0039 & 0.0370 & -0.1054 & 0.9161 \\
PC 5 & 0.0259 & 0.0424 & 0.6108 & 0.5414 \\
PC 6 & 0.0029 & 0.0570 & 0.0502 & 0.9600 \\
PC 7 & -0.0647 & 0.0397 & -1.6290 & 0.1034 \\
PC 8 & 0.0182 & 0.0779 & 0.2337 & 0.8153 \\
\hline B. smooth terms & edf & Ref.df & F-value & p-value \\
Location Smooth & 196.4300 & 589.0000 & 1.4030 & $<0.0001$ \\
Year Smooth & 12.8385 & 19.0000 & 264.7959 & $<0.0001$ \\
Station Random Effect & 4.6787 & 7.0000 & 2.8846 & 0.3412 \\
\hline
\end{tabular}

Table A3.35: Best productivity model for the Common Yellowthroat (Geothlypis trichas). The model was a quasi-Poisson GAMM with log link function. Based on AIC/QIC, support for including the noise level as a predictor of productivity was: none; support for including the standard deviation of noise was: none. 


\begin{tabular}{lrrrr}
\hline A. parametric coefficients & Estimate & Std. Error & t-value & p-value \\
Intercept & -6.2324 & 1.0356 & -6.0180 & $<0.0001$ \\
Noise Level & 0.0246 & 0.0270 & 0.9116 & 0.3620 \\
PC 1 & 0.0881 & 0.0835 & 1.0550 & 0.2915 \\
PC 2 & 0.2965 & 0.0707 & 4.1941 & $<0.0001$ \\
PC 3 & 0.0797 & 0.0954 & 0.8361 & 0.4031 \\
PC 4 & -0.0710 & 0.0613 & -1.1594 & 0.2464 \\
PC 5 & -0.0701 & 0.0697 & -1.0066 & 0.3142 \\
PC 6 & 0.0306 & 0.0833 & 0.3680 & 0.7129 \\
PC 7 & 0.0382 & 0.0683 & 0.5594 & 0.5759 \\
PC 8 & 0.0919 & 0.1089 & 0.8436 & 0.3989 \\
\hline B. smooth terms & edf & Ref.df & F-value & p-value \\
Location Smooth & 13.1816 & 19.0000 & 30975.2207 & $<0.0001$ \\
Year Smooth & 6.1323 & 7.0000 & 1614.8061 & $<0.0001$ \\
Station Random Effect & 513.7181 & 608.0000 & 17.2780 & $<0.0001$ \\
\hline
\end{tabular}

Table A3.36: Best abundance model for the Common Yellowthroat (Geothlypis trichas). The model was a quasi-Poisson GAMM with log link function. Based on AIC/QIC, support for including the noise level as a predictor of abundance was: weak; support for including the standard deviation of noise was: none.

\begin{tabular}{lrrrr}
\hline A. parametric coefficients & Estimate & Std. Error & t-value & p-value \\
Intercept & 0.8116 & 1.4555 & 0.5576 & 0.5772 \\
Noise Level & -0.0396 & 0.0415 & -0.9546 & 0.3399 \\
PC 1 & 0.1964 & 0.1807 & 1.0869 & 0.2773 \\
PC 2 & -0.1623 & 0.1507 & -1.0765 & 0.2819 \\
PC 3 & 0.0534 & 0.1206 & 0.4427 & 0.6581 \\
PC 4 & -0.4297 & 0.2722 & -1.5785 & 0.1147 \\
PC 5 & 0.5889 & 0.3240 & 1.8173 & 0.0694 \\
PC 6 & -0.3108 & 0.1999 & -1.5544 & 0.1203 \\
PC 7 & 0.7529 & 0.2551 & 2.9508 & 0.0032 \\
PC 8 & 0.1370 & 0.3321 & 0.4126 & 0.6799 \\
\hline B. smooth terms & edf & Ref.df & F-value & p-value \\
Location Smooth & 87.3808 & 166.0000 & 4.8069 & $<0.0001$ \\
Year Smooth & 1.5232 & 19.0000 & 13.5513 & 0.0592 \\
Station Random Effect & 4.1431 & 7.0000 & 10.0300 & 0.0002 \\
\hline
\end{tabular}

Table A3.37: Best productivity model for the Dark-eyed Junco (Junco hyemalis). The model was a quasi-Poisson GAMM with log link function. Based on AIC/QIC, support for including the noise level as a predictor of productivity was: weak; support for including the standard deviation of noise was: none. 


\begin{tabular}{lrrrr}
\hline A. parametric coefficients & Estimate & Std. Error & t-value & p-value \\
Intercept & -5.5723 & 0.1962 & -28.4078 & $<0.0001$ \\
PC 1 & -0.6625 & 0.2017 & -3.2848 & 0.0010 \\
PC 2 & -0.5440 & 0.1931 & -2.8164 & 0.0049 \\
PC 3 & -0.2985 & 0.1707 & -1.7484 & 0.0805 \\
PC 4 & -0.3588 & 0.2853 & -1.2576 & 0.2087 \\
PC 5 & 0.5446 & 0.3487 & 1.5619 & 0.1185 \\
PC 6 & -0.5210 & 0.2387 & -2.1829 & 0.0292 \\
PC 7 & -0.4009 & 0.3236 & -1.2390 & 0.2155 \\
PC 8 & -0.1320 & 0.4350 & -0.3034 & 0.7616 \\
\hline B. smooth terms & edf & Ref.df & F-value & p-value \\
Location Smooth & 8.7611 & 19.0000 & 8632.7063 & 0.0007 \\
Year Smooth & 4.1625 & 7.0000 & 80.8565 & $<0.0001$ \\
Station Random Effect & 148.2819 & 186.0000 & 12.8068 & $<0.0001$ \\
\hline
\end{tabular}

Table A3.38: Best abundance model for the Dark-eyed Junco (Junco hyemalis). The model was a quasi-Poisson GAMM with log link function. Based on AIC/QIC, support for including the noise level as a predictor of abundance was: none; support for including the standard deviation of noise was: none.

\begin{tabular}{lrrrr}
\hline A. parametric coefficients & Estimate & Std. Error & t-value & p-value \\
Intercept & -1.1962 & 2.4734 & -0.4836 & 0.6288 \\
Noise Level & -0.0416 & 0.0727 & -0.5717 & 0.5677 \\
PC 1 & -0.2133 & 0.4730 & -0.4509 & 0.6521 \\
PC 2 & 0.0129 & 0.3045 & 0.0424 & 0.9662 \\
PC 3 & -0.1553 & 0.1713 & -0.9063 & 0.3650 \\
PC 4 & 0.1478 & 0.3015 & 0.4902 & 0.6241 \\
PC 5 & 0.3208 & 0.2524 & 1.2708 & 0.2041 \\
PC 6 & 0.2181 & 0.2133 & 1.0224 & 0.3068 \\
PC 7 & -0.0591 & 0.3036 & -0.1948 & 0.8456 \\
PC 8 & -0.4577 & 0.8653 & -0.5290 & 0.5969 \\
\hline B. smooth terms & edf & Ref.df & F-value & p-value \\
Location Smooth & 46.6119 & 174.0000 & 0.7285 & $<0.0001$ \\
Year Smooth & 0.0019 & 19.0000 & 0.0001 & 0.6193 \\
Station Random Effect & 0.0012 & 7.0000 & 0.0001 & 0.5061 \\
\hline
\end{tabular}

Table A3.39: Best productivity model for the Dusky Flycatcher (Empidonax oberholseri). The model was a quasi-Poisson GAMM with log link function. Based on AIC/QIC, support for including the noise level as a predictor of productivity was: weak; support for including the standard deviation of noise was: none. 


\begin{tabular}{lrrrr}
\hline A. parametric coefficients & Estimate & Std. Error & t-value & p-value \\
Intercept & -3.6109 & 2.3117 & -1.5620 & 0.1185 \\
Noise Level & -0.0735 & 0.0673 & -1.0925 & 0.2748 \\
PC 1 & -0.2475 & 0.2661 & -0.9302 & 0.3524 \\
PC 2 & -0.2639 & 0.2016 & -1.3090 & 0.1907 \\
PC 3 & 0.1465 & 0.1503 & 0.9742 & 0.3301 \\
PC 4 & -0.0026 & 0.2111 & -0.0123 & 0.9902 \\
PC 5 & -0.0516 & 0.1823 & -0.2830 & 0.7772 \\
PC 6 & -0.1897 & 0.1919 & -0.9887 & 0.3230 \\
PC 7 & -0.0185 & 0.3109 & -0.0595 & 0.9526 \\
PC 8 & 0.0709 & 0.5260 & 0.1348 & 0.8928 \\
\hline B. smooth terms & edf & Ref.df & F-value & p-value \\
Location Smooth & 6.1217 & 19.0000 & 760.8768 & 0.1128 \\
Year Smooth & 1.7281 & 7.0000 & 6.9451 & 0.0008 \\
Station Random Effect & 140.6265 & 176.0000 & 10.7241 & $<0.0001$ \\
\hline
\end{tabular}

Table A3.40: Best abundance model for the Dusky Flycatcher (Empidonax oberholseri). The model was a quasi-Poisson GAMM with log link function. Based on AIC/QIC, support for including the noise level as a predictor of abundance was: weak; support for including the standard deviation of noise was: none. 


\begin{tabular}{lrrrr}
\hline A. parametric coefficients & Estimate & Std. Error & t-value & p-value \\
Intercept & 0.2517 & 0.3271 & 0.7695 & 0.4422 \\
Noise Standard Deviation & -0.7839 & 0.3766 & -2.0813 & 0.0382 \\
PC 1 & -0.0791 & 0.2787 & -0.2836 & 0.7769 \\
PC 2 & 0.1828 & 0.2052 & 0.8909 & 0.3737 \\
PC 3 & 0.2305 & 0.1362 & 1.6927 & 0.0915 \\
PC 4 & 0.1135 & 0.1688 & 0.6724 & 0.5019 \\
PC 5 & -0.0620 & 0.2204 & -0.2811 & 0.7788 \\
PC 6 & 0.0085 & 0.1727 & 0.0491 & 0.9608 \\
PC 7 & 0.0381 & 0.1481 & 0.2571 & 0.7972 \\
PC 8 & -0.2513 & 0.4443 & -0.5656 & 0.5721 \\
\hline B. smooth terms & edf & Ref.df & F-value & p-value \\
Location Smooth & 38.6779 & 122.0000 & 0.8640 & $<0.0001$ \\
Year Smooth & 0.0002 & 19.0000 & 0.0000 & 0.7764 \\
Station Random Effect & 1.4025 & 7.0000 & 1.1520 & 0.0644 \\
\hline
\end{tabular}

Table A3.41: Best productivity model for the Eastern Bluebird (Sialia sialis). The model was a quasi-Poisson GAMM with log link function. Based on AIC/QIC, support for including the noise level as a predictor of productivity was: none; support for including the standard deviation of noise was: strong. 


\begin{tabular}{lrrrr}
\hline A. parametric coefficients & Estimate & Std. Error & t-value & p-value \\
Intercept & -6.9718 & 0.3563 & -19.5662 & $<0.0001$ \\
Noise Standard Deviation & -0.2963 & 0.3573 & -0.8293 & 0.4071 \\
PC 1 & 0.2960 & 0.3056 & 0.9684 & 0.3331 \\
PC 2 & 0.0955 & 0.2236 & 0.4272 & 0.6693 \\
PC 3 & 0.1264 & 0.1811 & 0.6981 & 0.4852 \\
PC 4 & 0.0292 & 0.2249 & 0.1298 & 0.8968 \\
PC 5 & -0.1168 & 0.2707 & -0.4315 & 0.6662 \\
PC 6 & 0.1156 & 0.2161 & 0.5351 & 0.5927 \\
PC 7 & 0.0696 & 0.1939 & 0.3588 & 0.7198 \\
PC 8 & 0.0447 & 0.4955 & 0.0903 & 0.9281 \\
\hline B. smooth terms & edf & Ref.df & F-value & p-value \\
Location Smooth & 0.0003 & 19.0000 & 0.0000 & 0.7622 \\
Year Smooth & 5.3904 & 7.0000 & 14.8540 & 0.0085 \\
Station Random Effect & 100.6254 & 142.0000 & 2.8797 & $<0.0001$ \\
\hline
\end{tabular}

Table A3.42: Best abundance model for the Eastern Bluebird (Sialia sialis). The model was a quasi-Poisson GAMM with log link function. Based on AIC/QIC, support for including the noise level as a predictor of abundance was: none; support for including the standard deviation of noise was: strong.

\begin{tabular}{lrrrr}
\hline A. parametric coefficients & Estimate & Std. Error & t-value & p-value \\
Intercept & -2972.7913 & 61676288.1524 & -0.0000 & 1.0000 \\
Noise Level & 0.6374 & 0.4846 & 1.3155 & 0.1903 \\
Noise Standard Deviation & -0.2319 & 0.9485 & -0.2445 & 0.8072 \\
PC 1 & 572.0390 & 12277144.3986 & 0.0000 & 1.0000 \\
PC 2 & -819.1095 & 17366174.7207 & -0.0000 & 1.0000 \\
PC 3 & -3322.2675 & 69808776.8317 & -0.0000 & 1.0000 \\
PC 4 & -523.2120 & 10931472.7711 & -0.0000 & 1.0000 \\
PC 5 & 649.8152 & 13670888.4804 & 0.0000 & 1.0000 \\
PC 6 & 2331.5805 & 48967916.2447 & 0.0000 & 1.0000 \\
PC 7 & -1097.7646 & 23107693.2602 & -0.0000 & 1.0000 \\
PC 8 & 220.6682 & 4913826.2778 & 0.0000 & 1.0000 \\
\hline B. smooth terms & edf & Ref.df & F-value & p-value \\
Location Smooth & 0.0000 & 15.0000 & 0.0000 & 0.1222 \\
Year Smooth & 0.0000 & 7.0000 & 0.0000 & 0.5340 \\
Station Random Effect & 3.3106 & 15.0000 & 0.4425 & 0.0327 \\
\hline
\end{tabular}

Table A3.43: Best abundance model for the Eastern Meadowlark (Sturnella magna). The model was a quasi-Poisson GAMM with log link function. Based on AIC/QIC, support for including the noise level as a predictor of abundance was: strong; support for including the standard deviation of noise was: strong. 


\begin{tabular}{lrrrr}
\hline A. parametric coefficients & Estimate & Std. Error & t-value & p-value \\
Intercept & -8.5060 & 9.8321 & -0.8651 & 0.3879 \\
Noise Level & 0.2070 & 0.2235 & 0.9263 & 0.3553 \\
Noise Standard Deviation & -0.4910 & 0.8288 & -0.5925 & 0.5542 \\
PC 1 & 1.7284 & 11.8125 & 0.1463 & 0.8838 \\
PC 2 & -1.2648 & 6.7869 & -0.1864 & 0.8523 \\
PC 3 & 0.4197 & 1.8143 & 0.2313 & 0.8173 \\
PC 4 & -1.2980 & 2.8948 & -0.4484 & 0.6543 \\
PC 5 & 2.3741 & 2.1446 & 1.1070 & 0.2695 \\
PC 6 & -1.3798 & 3.0971 & -0.4455 & 0.6564 \\
PC 7 & 0.7407 & 1.8055 & 0.4102 & 0.6820 \\
PC 8 & 3.5497 & 16.8204 & 0.2110 & 0.8331 \\
\hline B. smooth terms & edf & Ref.df & F-value & p-value \\
Location Smooth & 13.4441 & 58.0000 & 0.6277 & $<0.0001$ \\
Year Smooth & 0.0001 & 19.0000 & 0.0000 & 0.6218 \\
Station Random Effect & 0.0000 & 7.0000 & 0.0000 & 1.0000 \\
\hline
\end{tabular}

Table A3.44: Best productivity model for the Evening Grosbeak (Coccothraustes vespertinus). The model was a quasi-Poisson GAMM with log link function. Based on AIC/QIC, support for including the noise level as a predictor of productivity was: strong; support for including the standard deviation of noise was: strong.

\begin{tabular}{lrrrr}
\hline A. parametric coefficients & Estimate & Std. Error & t-value & p-value \\
Intercept & -7.6580 & 6.5219 & -1.1742 & 0.2406 \\
Noise Standard Deviation & 0.4699 & 0.5605 & 0.8384 & 0.4020 \\
PC 1 & 0.5929 & 11.8231 & 0.0502 & 0.9600 \\
PC 2 & -1.2578 & 6.7572 & -0.1861 & 0.8524 \\
PC 3 & 0.5714 & 1.7883 & 0.3195 & 0.7494 \\
PC 4 & 0.5402 & 2.5070 & 0.2155 & 0.8294 \\
PC 5 & -0.1425 & 1.1389 & -0.1251 & 0.9005 \\
PC 6 & -0.1953 & 2.8658 & -0.0681 & 0.9457 \\
PC 7 & -0.3825 & 1.4461 & -0.2645 & 0.7914 \\
PC 8 & 2.1182 & 15.8795 & 0.1334 & 0.8939 \\
\hline B. smooth terms & edf & Ref.df & F-value & p-value \\
Location Smooth & 0.0012 & 19.0000 & 0.0000 & 0.8365 \\
Year Smooth & 5.5842 & 7.0000 & 19.4798 & $<0.0001$ \\
Station Random Effect & 45.5433 & 61.0000 & 7.7654 & $<0.0001$ \\
\hline
\end{tabular}

Table A3.45: Best abundance model for the Evening Grosbeak (Coccothraustes vespertinus). The model was a quasi-Poisson GAMM with log link function. Based on AIC/QIC, support for including the noise level as a predictor of abundance was: none; support for including the standard deviation of noise was: weak. 


\begin{tabular}{lrrrr}
\hline A. parametric coefficients & Estimate & Std. Error & t-value & p-value \\
Intercept & -5.9585 & 1.6813 & -3.5441 & 0.0004 \\
Noise Standard Deviation & 1.0216 & 1.7252 & 0.5922 & 0.5539 \\
PC 1 & -0.1685 & 0.8455 & -0.1994 & 0.8420 \\
PC 2 & 0.2537 & 0.8307 & 0.3054 & 0.7602 \\
PC 3 & 0.2254 & 0.8466 & 0.2663 & 0.7901 \\
PC 4 & -0.1467 & 1.1013 & -0.1332 & 0.8941 \\
PC 5 & 0.3846 & 1.2851 & 0.2993 & 0.7648 \\
PC 6 & -0.3905 & 0.9912 & -0.3939 & 0.6937 \\
PC 7 & 0.5423 & 0.9002 & 0.6024 & 0.5471 \\
PC 8 & -0.6178 & 1.7002 & -0.3634 & 0.7164 \\
\hline B. smooth terms & edf & Ref.df & F-value & p-value \\
Location Smooth & 105.2900 & 261.0000 & 0.3022 & 0.9917 \\
Year Smooth & 0.0000 & 19.0000 & 0.0000 & 1.0000 \\
Station Random Effect & 6.6562 & 7.0000 & 81.8866 & 0.3197 \\
\hline
\end{tabular}

Table A3.46: Best productivity model for the Great Crested Flycatcher (Myiarchus crinitus). The model was a quasi-Poisson GAMM with log link function. Based on AIC/QIC, support for including the noise level as a predictor of productivity was: none; support for including the standard deviation of noise was: strong.

\begin{tabular}{lrrrr}
\hline A. parametric coefficients & Estimate & Std. Error & t-value & p-value \\
Intercept & -5.1106 & 1.2350 & -4.1380 & $<0.0001$ \\
Noise Level & -0.0479 & 0.0312 & -1.5329 & 0.1254 \\
PC 1 & 0.2517 & 0.0895 & 2.8107 & 0.0050 \\
PC 2 & 0.1454 & 0.0793 & 1.8343 & 0.0667 \\
PC 3 & 0.0631 & 0.1375 & 0.4586 & 0.6466 \\
PC 4 & 0.1091 & 0.1016 & 1.0736 & 0.2831 \\
PC 5 & -0.1393 & 0.1155 & -1.2063 & 0.2278 \\
PC 6 & 0.2013 & 0.1049 & 1.9191 & 0.0551 \\
PC 7 & -0.0392 & 0.0920 & -0.4256 & 0.6704 \\
PC 8 & 0.0139 & 0.0942 & 0.1479 & 0.8824 \\
\hline B. smooth terms & edf & Ref.df & F-value & p-value \\
Location Smooth & 10.8623 & 19.0000 & 77.0680 & $<0.0001$ \\
Year Smooth & 0.0008 & 7.0000 & 0.0000 & 0.8331 \\
Station Random Effect & 168.0046 & 272.0000 & 2.2700 & $<0.0001$ \\
\hline
\end{tabular}

Table A3.47: Best abundance model for the Great Crested Flycatcher (Myiarchus crinitus). The model was a quasi-Poisson GAMM with log link function. Based on AIC/QIC, support for including the noise level as a predictor of abundance was: strong; support for including the standard deviation of noise was: none. 


\begin{tabular}{lrrrr}
\hline A. parametric coefficients & Estimate & Std. Error & t-value & p-value \\
Intercept & -0.8355 & 0.4623 & -1.8073 & 0.0712 \\
PC 1 & -1.0513 & 0.4999 & -2.1030 & 0.0359 \\
PC 2 & 0.2179 & 0.4007 & 0.5437 & 0.5868 \\
PC 3 & 0.1258 & 0.3140 & 0.4008 & 0.6887 \\
PC 4 & 0.0010 & 0.4563 & 0.0021 & 0.9983 \\
PC 5 & -0.5405 & 0.5340 & -1.0122 & 0.3119 \\
PC 6 & -0.3590 & 0.4527 & -0.7931 & 0.4280 \\
PC 7 & 0.9127 & 0.3654 & 2.4978 & 0.0128 \\
PC 8 & -1.8630 & 0.8824 & -2.1112 & 0.0352 \\
\hline B. smooth terms & edf & Ref.df & F-value & p-value \\
Location Smooth & 46.1816 & 117.0000 & 2.5648 & $<0.0001$ \\
Year Smooth & 2.1210 & 19.0000 & 3.1654 & 0.2438 \\
Station Random Effect & 4.2618 & 7.0000 & 9.2539 & $<0.0001$ \\
\hline
\end{tabular}

Table A3.48: Best productivity model for the Golden-crowned Kinglet (Regulus satrapa). The model was a quasi-Poisson GAMM with log link function. Based on AIC/QIC, support for including the noise level as a predictor of productivity was: none; support for including the standard deviation of noise was: none.

\begin{tabular}{lrrrr}
\hline A. parametric coefficients & Estimate & Std. Error & t-value & p-value \\
Intercept & -7.4683 & 0.4529 & -16.4884 & $<0.0001$ \\
Noise Standard Deviation & 0.6425 & 0.3475 & 1.8487 & 0.0647 \\
PC 1 & 0.1183 & 0.4118 & 0.2872 & 0.7740 \\
PC 2 & -0.2743 & 0.2894 & -0.9478 & 0.3434 \\
PC 3 & -0.2361 & 0.2720 & -0.8678 & 0.3856 \\
PC 4 & 0.2053 & 0.3143 & 0.6531 & 0.5137 \\
PC 5 & -0.1312 & 0.3391 & -0.3871 & 0.6988 \\
PC 6 & -0.3887 & 0.3549 & -1.0953 & 0.2735 \\
PC 7 & 0.0019 & 0.2724 & 0.0068 & 0.9946 \\
PC 8 & -0.0088 & 0.8371 & -0.0105 & 0.9917 \\
\hline B. smooth terms & edf & Ref.df & F-value & p-value \\
Location Smooth & 13.8307 & 19.0000 & 1354.6830 & 0.0002 \\
Year Smooth & 4.3945 & 7.0000 & 7.4189 & $<0.0001$ \\
Station Random Effect & 94.9795 & 138.0000 & 6.5432 & $<0.0001$ \\
\hline
\end{tabular}

Table A3.49: Best abundance model for the Golden-crowned Kinglet (Regulus satrapa). The model was a quasi-Poisson GAMM with log link function. Based on AIC/QIC, support for including the noise level as a predictor of abundance was: none; support for including the standard deviation of noise was: strong. 


\begin{tabular}{lrrrr}
\hline A. parametric coefficients & Estimate & Std. Error & t-value & p-value \\
Intercept & -1.6069 & 0.1368 & -11.7450 & $<0.0001$ \\
Noise Standard Deviation & 0.1922 & 0.1211 & 1.5878 & 0.1125 \\
PC 1 & 0.0843 & 0.0409 & 2.0608 & 0.0395 \\
PC 2 & 0.0379 & 0.0532 & 0.7128 & 0.4761 \\
PC 3 & 0.1388 & 0.0935 & 1.4839 & 0.1380 \\
PC 4 & -0.1065 & 0.0608 & -1.7516 & 0.0800 \\
PC 5 & 0.0308 & 0.0736 & 0.4187 & 0.6755 \\
PC 6 & 0.0095 & 0.0794 & 0.1201 & 0.9044 \\
PC 7 & 0.0947 & 0.0653 & 1.4502 & 0.1472 \\
PC 8 & -0.0617 & 0.0615 & -1.0023 & 0.3163 \\
\hline B. smooth terms & edf & Ref.df & F-value & p-value \\
Location Smooth & 166.4613 & 387.0000 & 2.8805 & $<0.0001$ \\
Year Smooth & 4.8990 & 19.0000 & 68.1787 & 0.0146 \\
Station Random Effect & 1.1626 & 7.0000 & 4.1632 & 0.0679 \\
\hline
\end{tabular}

Table A3.50: Best productivity model for the Gray Catbird (Dumetella carolinensis). The model was a quasi-Poisson GAMM with log link function. Based on AIC/QIC, support for including the noise level as a predictor of productivity was: none; support for including the standard deviation of noise was: strong.

\begin{tabular}{lrrrr}
\hline A. parametric coefficients & Estimate & Std. Error & t-value & p-value \\
Intercept & -4.9919 & 0.1876 & -26.6020 & $<0.0001$ \\
Noise Standard Deviation & 0.2173 & 0.1823 & 1.1921 & 0.2333 \\
PC 1 & 0.2967 & 0.0649 & 4.5699 & $<0.0001$ \\
PC 2 & 0.2130 & 0.0840 & 2.5353 & 0.0113 \\
PC 3 & 0.2540 & 0.1321 & 1.9231 & 0.0546 \\
PC 4 & -0.3250 & 0.1004 & -3.2363 & 0.0012 \\
PC 5 & 0.0183 & 0.1166 & 0.1567 & 0.8755 \\
PC 6 & 0.0631 & 0.1229 & 0.5131 & 0.6079 \\
PC 7 & 0.2366 & 0.1015 & 2.3312 & 0.0198 \\
PC 8 & -0.0015 & 0.1095 & -0.0133 & 0.9894 \\
\hline B. smooth terms & edf & Ref.df & F-value & p-value \\
Location Smooth & 14.8522 & 19.0000 & 33031.6034 & $<0.0001$ \\
Year Smooth & 5.0077 & 7.0000 & 482.4494 & $<0.0001$ \\
Station Random Effect & 324.9407 & 395.0000 & 16.5162 & $<0.0001$ \\
\hline
\end{tabular}

Table A3.51: Best abundance model for the Gray Catbird (Dumetella carolinensis). The model was a quasi-Poisson GAMM with log link function. Based on AIC/QIC, support for including the noise level as a predictor of abundance was: none; support for including the standard deviation of noise was: weak. 


\begin{tabular}{lrrrr}
\hline A. parametric coefficients & Estimate & Std. Error & t-value & p-value \\
Intercept & -1977.5077 & 28085588.9940 & -0.0001 & 0.9999 \\
Noise Level & 0.0949 & 0.2724 & 0.3485 & 0.7281 \\
Noise Standard Deviation & -0.7480 & 2.4219 & -0.3088 & 0.7580 \\
PC 1 & -3472.4891 & 49450410.5066 & -0.0001 & 0.9999 \\
PC 2 & 1944.1071 & 27692425.1635 & 0.0001 & 0.9999 \\
PC 3 & -527.1750 & 7516880.1482 & -0.0001 & 0.9999 \\
PC 4 & -763.5831 & 10891359.2795 & -0.0001 & 0.9999 \\
PC 5 & -169.3485 & 2431859.7519 & -0.0001 & 0.9999 \\
PC 6 & 753.8032 & 10724774.1527 & 0.0001 & 0.9999 \\
PC 7 & 416.7894 & 5941793.3038 & 0.0001 & 0.9999 \\
PC 8 & -4472.2331 & 63707666.5731 & -0.0001 & 0.9999 \\
\hline B. smooth terms & edf & Ref.df & F-value & p-value \\
Location Smooth & 0.0000 & 32.0000 & 0.0000 & 0.6983 \\
Year Smooth & 3.8706 & 19.0000 & 1.0754 & 0.0001 \\
Station Random Effect & 1.9745 & 7.0000 & 0.4765 & 0.1707 \\
\hline
\end{tabular}

Table A3.52: Best productivity model for the Gray Flycatcher (Empidonax wrightii). The model was a quasi-Poisson GAMM with log link function. Based on AIC/QIC, support for including the noise level as a predictor of productivity was: strong; support for including the standard deviation of noise was: strong.

\begin{tabular}{lrrrr}
\hline A. parametric coefficients & Estimate & Std. Error & t-value & p-value \\
Intercept & -4.0092 & 3.5361 & -1.1338 & 0.2574 \\
Noise Level & -0.1243 & 0.1169 & -1.0628 & 0.2884 \\
Noise Standard Deviation & 0.4627 & 0.8058 & 0.5743 & 0.5660 \\
PC 1 & -1.2021 & 1.1739 & -1.0241 & 0.3063 \\
PC 2 & 0.7951 & 1.0198 & 0.7796 & 0.4360 \\
PC 3 & -0.2671 & 0.5958 & -0.4482 & 0.6542 \\
PC 4 & -0.1850 & 0.7343 & -0.2519 & 0.8012 \\
PC 5 & 0.2691 & 0.3217 & 0.8366 & 0.4032 \\
PC 6 & 0.5565 & 0.6093 & 0.9132 & 0.3615 \\
PC 7 & 0.1474 & 1.2433 & 0.1185 & 0.9057 \\
PC 8 & -1.0044 & 2.1619 & -0.4646 & 0.6424 \\
\hline B. smooth terms & edf & Ref.df & F-value & p-value \\
Location Smooth & 1.6134 & 19.0000 & 4.4547 & 0.0597 \\
Year Smooth & 3.1822 & 7.0000 & 0.7765 & 0.2196 \\
Station Random Effect & 23.8302 & 40.0000 & 1.9369 & $<0.0001$ \\
\hline
\end{tabular}

Table A3.53: Best abundance model for the Gray Flycatcher (Empidonax wrightii). The model was a quasi-Poisson GAMM with log link function. Based on AIC/QIC, support for including the noise level as a predictor of abundance was: weak; support for including the standard deviation of noise was: weak. 


\begin{tabular}{lrrrr}
\hline A. parametric coefficients & Estimate & Std. Error & t-value & p-value \\
Intercept & 0.1298 & 5.6856 & 0.0228 & 0.9818 \\
Noise Level & -0.1256 & 0.1498 & -0.8386 & 0.4019 \\
Noise Standard Deviation & 0.1106 & 0.4053 & 0.2729 & 0.7850 \\
PC 1 & -2.1137 & 3.6469 & -0.5796 & 0.5624 \\
PC 2 & 0.0757 & 2.0637 & 0.0367 & 0.9707 \\
PC 3 & -0.3813 & 0.6068 & -0.6284 & 0.5299 \\
PC 4 & -2.1069 & 1.1642 & -1.8098 & 0.0707 \\
PC 5 & 1.1211 & 0.9213 & 1.2168 & 0.2240 \\
PC 6 & -0.7511 & 0.9705 & -0.7739 & 0.4392 \\
PC 7 & 0.0779 & 0.9792 & 0.0795 & 0.9366 \\
PC 8 & -2.7226 & 4.9174 & -0.5537 & 0.5800 \\
\hline B. smooth terms & edf & Ref.df & F-value & p-value \\
Location Smooth & 60.8421 & 140.0000 & 2.9254 & $<0.0001$ \\
Year Smooth & 0.0010 & 19.0000 & 0.0000 & 0.6960 \\
Station Random Effect & 4.6296 & 7.0000 & 3.7084 & 0.0869 \\
\hline
\end{tabular}

Table A3.54: Best productivity model for the Hammond's Flycatcher (Empidonax hammondii). The model was a quasi-Poisson GAMM with log link function. Based on AIC/QIC, support for including the noise level as a predictor of productivity was: weak; support for including the standard deviation of noise was: weak.

\begin{tabular}{lrrrr}
\hline A. parametric coefficients & Estimate & Std. Error & t-value & p-value \\
Intercept & -6.9246 & 0.3218 & -21.5211 & $<0.0001$ \\
Noise Standard Deviation & 0.4976 & 0.2575 & 1.9326 & 0.0534 \\
PC 1 & -0.3397 & 0.2443 & -1.3902 & 0.1647 \\
PC 2 & 0.0202 & 0.2074 & 0.0974 & 0.9224 \\
PC 3 & 0.0730 & 0.1684 & 0.4337 & 0.6646 \\
PC 4 & -0.0409 & 0.2405 & -0.1699 & 0.8651 \\
PC 5 & 0.2038 & 0.2243 & 0.9088 & 0.3636 \\
PC 6 & -0.2940 & 0.2196 & -1.3393 & 0.1807 \\
PC 7 & -0.4467 & 0.2960 & -1.5090 & 0.1315 \\
PC 8 & -0.0996 & 0.7208 & -0.1381 & 0.8902 \\
\hline B. smooth terms & edf & Ref.df & F-value & p-value \\
Location Smooth & 5.6307 & 19.0000 & 400.2779 & 0.0002 \\
Year Smooth & 3.6186 & 7.0000 & 15.0644 & $<0.0001$ \\
Station Random Effect & 113.6663 & 148.0000 & 5.3190 & $<0.0001$ \\
\hline
\end{tabular}

Table A3.55: Best abundance model for the Hammond's Flycatcher (Empidonax hammondii). The model was a quasi-Poisson GAMM with log link function. Based on AIC/QIC, support for including the noise level as a predictor of abundance was: none; support for including the standard deviation of noise was: weak. 


\begin{tabular}{lrrrr}
\hline A. parametric coefficients & Estimate & Std. Error & t-value & p-value \\
Intercept & 0.9433 & 2.2089 & 0.4270 & 0.6695 \\
Noise Level & -0.0787 & 0.0633 & -1.2437 & 0.2139 \\
PC 1 & 0.0470 & 0.2470 & 0.1903 & 0.8491 \\
PC 2 & -0.0225 & 0.1757 & -0.1281 & 0.8981 \\
PC 3 & -0.1219 & 0.1571 & -0.7757 & 0.4381 \\
PC 4 & -0.0738 & 0.1858 & -0.3970 & 0.6915 \\
PC 5 & 0.1731 & 0.1884 & 0.9188 & 0.3584 \\
PC 6 & 0.2305 & 0.2121 & 1.0872 & 0.2772 \\
PC 7 & 0.0991 & 0.1490 & 0.6653 & 0.5060 \\
PC 8 & -0.5199 & 0.4613 & -1.1270 & 0.2600 \\
\hline B. smooth terms & edf & Ref.df & F-value & p-value \\
Location Smooth & 74.9000 & 228.0000 & 0.7392 & $<0.0001$ \\
Year Smooth & 5.5922 & 19.0000 & 14.0722 & $<0.0001$ \\
Station Random Effect & 0.5521 & 7.0000 & 0.3357 & 0.1795 \\
\hline
\end{tabular}

Table A3.56: Best productivity model for the Hermit Thrush (Catharus guttatus). The model was a quasi-Poisson GAMM with log link function. Based on AIC/QIC, support for including the noise level as a predictor of productivity was: strong; support for including the standard deviation of noise was: none.

\begin{tabular}{lrrrr}
\hline A. parametric coefficients & Estimate & Std. Error & t-value & p-value \\
Intercept & -3.2609 & 2.3848 & -1.3674 & 0.1716 \\
Noise Level & -0.0987 & 0.0695 & -1.4201 & 0.1557 \\
Noise Standard Deviation & 0.0391 & 0.2822 & 0.1386 & 0.8898 \\
PC 1 & -0.0687 & 0.1905 & -0.3608 & 0.7183 \\
PC 2 & -0.2417 & 0.1421 & -1.7006 & 0.0892 \\
PC 3 & -0.1177 & 0.1603 & -0.7340 & 0.4630 \\
PC 4 & 0.0703 & 0.1787 & 0.3934 & 0.6941 \\
PC 5 & 0.2639 & 0.1813 & 1.4552 & 0.1457 \\
PC 6 & 0.1892 & 0.2241 & 0.8444 & 0.3985 \\
PC 7 & 0.0329 & 0.1522 & 0.2164 & 0.8287 \\
PC 8 & 0.1984 & 0.3752 & 0.5288 & 0.5970 \\
\hline B. smooth terms & edf & Ref.df & F-value & p-value \\
Location Smooth & 12.7934 & 19.0000 & 1578.6367 & 0.0011 \\
Year Smooth & 2.2617 & 7.0000 & 2.9104 & 0.2369 \\
Station Random Effect & 187.8392 & 246.0000 & 5.6911 & $<0.0001$ \\
\hline
\end{tabular}

Table A3.57: Best abundance model for the Hermit Thrush (Catharus guttatus). The model was a quasi-Poisson GAMM with log link function. Based on AIC/QIC, support for including the noise level as a predictor of abundance was: strong; support for including the standard deviation of noise was: weak. 


\begin{tabular}{lrrrr}
\hline A. parametric coefficients & Estimate & Std. Error & t-value & p-value \\
Intercept & -0.5561 & 0.2440 & -2.2795 & 0.0230 \\
Noise Standard Deviation & 0.1813 & 0.1829 & 0.9913 & 0.3219 \\
PC 1 & 0.1246 & 0.0901 & 1.3827 & 0.1673 \\
PC 2 & 0.0048 & 0.1022 & 0.0471 & 0.9624 \\
PC 3 & 0.0016 & 0.0735 & 0.0220 & 0.9824 \\
PC 4 & -0.0255 & 0.0839 & -0.3036 & 0.7615 \\
PC 5 & 0.1793 & 0.1035 & 1.7324 & 0.0837 \\
PC 6 & 0.0014 & 0.0853 & 0.0160 & 0.9872 \\
PC 7 & -0.0172 & 0.1098 & -0.1568 & 0.8755 \\
PC 8 & 0.0120 & 0.0833 & 0.1442 & 0.8854 \\
\hline B. smooth terms & edf & Ref.df & F-value & p-value \\
Location Smooth & 90.3872 & 205.0000 & 2.4188 & $<0.0001$ \\
Year Smooth & 0.0003 & 19.0000 & 0.0000 & 1.0000 \\
Station Random Effect & 0.0004 & 7.0000 & 0.0000 & 0.4120 \\
\hline
\end{tabular}

Table A3.58: Best productivity model for the House Finch (Haemorhous mexicanus). The model was a quasi-Poisson GAMM with log link function. Based on AIC/QIC, support for including the noise level as a predictor of productivity was: none; support for including the standard deviation of noise was: weak.

\begin{tabular}{lrrrr}
\hline A. parametric coefficients & Estimate & Std. Error & t-value & p-value \\
Intercept & -8.2585 & 1.2772 & -6.4661 & $<0.0001$ \\
Noise Level & 0.0280 & 0.0339 & 0.8242 & 0.4100 \\
PC 1 & 0.5837 & 0.1257 & 4.6437 & $<0.0001$ \\
PC 2 & 0.5201 & 0.1254 & 4.1463 & $<0.0001$ \\
PC 3 & 0.3322 & 0.1084 & 3.0647 & 0.0022 \\
PC 4 & -0.3046 & 0.0916 & -3.3241 & 0.0009 \\
PC 5 & -0.1073 & 0.1076 & -0.9975 & 0.3187 \\
PC 6 & -0.2256 & 0.1072 & -2.1058 & 0.0354 \\
PC 7 & 0.0384 & 0.1265 & 0.3031 & 0.7618 \\
PC 8 & 0.1939 & 0.1135 & 1.7093 & 0.0876 \\
\hline B. smooth terms & edf & Ref.df & F-value & p-value \\
Location Smooth & 2.8036 & 19.0000 & 113.1749 & 0.0066 \\
Year Smooth & 5.9394 & 7.0000 & 537.7151 & $<0.0001$ \\
Station Random Effect & 182.5558 & 241.0000 & 9.0364 & $<0.0001$ \\
\hline
\end{tabular}

Table A3.59: Best abundance model for the House Finch (Haemorhous mexicanus). The model was a quasi-Poisson GAMM with log link function. Based on AIC/QIC, support for including the noise level as a predictor of abundance was: weak; support for including the standard deviation of noise was: none. 


\begin{tabular}{lrrrr}
\hline A. parametric coefficients & Estimate & Std. Error & t-value & p-value \\
Intercept & -2.0424 & 0.7859 & -2.5988 & 0.0106 \\
Noise Standard Deviation & 0.5559 & 0.5273 & 1.0543 & 0.2940 \\
PC 1 & 0.0535 & 0.2703 & 0.1979 & 0.8434 \\
PC 2 & -0.1928 & 0.3423 & -0.5632 & 0.5744 \\
PC 3 & 0.1696 & 0.3159 & 0.5370 & 0.5923 \\
PC 4 & -0.3313 & 0.2649 & -1.2508 & 0.2136 \\
PC 5 & -0.0790 & 0.3750 & -0.2105 & 0.8336 \\
PC 6 & 0.3095 & 0.4779 & 0.6477 & 0.5185 \\
PC 7 & 0.1313 & 0.4711 & 0.2788 & 0.7809 \\
PC 8 & 0.0263 & 0.1794 & 0.1465 & 0.8838 \\
\hline B. smooth terms & edf & Ref.df & F-value & p-value \\
Location Smooth & 13.7906 & 57.0000 & 0.6624 & 0.0001 \\
Year Smooth & 0.0000 & 19.0000 & 0.0000 & 0.9938 \\
Station Random Effect & 0.8481 & 7.0000 & 2.2571 & 0.0424 \\
\hline
\end{tabular}

Table A3.60: Best productivity model for the House Sparrow (Passer domesticus). The model was a quasi-Poisson GAMM with log link function. Based on AIC/QIC, support for including the noise level as a predictor of productivity was: none; support for including the standard deviation of noise was: weak. 


\begin{tabular}{lrrrr}
\hline A. parametric coefficients & Estimate & Std. Error & t-value & p-value \\
Intercept & -8.0697 & 0.4143 & -19.4800 & $<0.0001$ \\
PC 1 & 0.5308 & 0.2067 & 2.5679 & 0.0105 \\
PC 2 & 0.1055 & 0.2475 & 0.4263 & 0.6700 \\
PC 3 & 0.1046 & 0.2567 & 0.4076 & 0.6837 \\
PC 4 & -0.2542 & 0.1970 & -1.2899 & 0.1976 \\
PC 5 & -0.3191 & 0.2798 & -1.1406 & 0.2545 \\
PC 6 & 0.0170 & 0.3882 & 0.0437 & 0.9651 \\
PC 7 & 0.1143 & 0.2529 & 0.4520 & 0.6514 \\
PC 8 & 0.0721 & 0.1753 & 0.4113 & 0.6810 \\
\hline B. smooth terms & edf & Ref.df & F-value & p-value \\
Location Smooth & 0.0004 & 19.0000 & 0.0000 & 0.5679 \\
Year Smooth & 4.6763 & 7.0000 & 11.5020 & 0.3038 \\
Station Random Effect & 53.2272 & 68.0000 & 5.6859 & $<0.0001$ \\
\hline
\end{tabular}

Table A3.61: Best abundance model for the House Sparrow (Passer domesticus). The model was a quasi-Poisson GAMM with log link function. Based on AIC/QIC, support for including the noise level as a predictor of abundance was: none; support for including the standard deviation of noise was: none.

\begin{tabular}{lrrrr}
\hline A. parametric coefficients & Estimate & Std. Error & t-value & p-value \\
Intercept & -1.5330 & 0.6730 & -2.2780 & 0.0229 \\
Noise Level & 0.0187 & 0.0180 & 1.0394 & 0.2988 \\
PC 1 & 0.0431 & 0.0780 & 0.5526 & 0.5806 \\
PC 2 & 0.1531 & 0.0602 & 2.5432 & 0.0111 \\
PC 3 & 0.0110 & 0.0479 & 0.2305 & 0.8178 \\
PC 4 & -0.0050 & 0.0574 & -0.0864 & 0.9312 \\
PC 5 & 0.0251 & 0.0652 & 0.3857 & 0.6998 \\
PC 6 & 0.0195 & 0.0534 & 0.3652 & 0.7151 \\
PC 7 & -0.0982 & 0.0648 & -1.5167 & 0.1296 \\
PC 8 & -0.2473 & 0.1217 & -2.0315 & 0.0424 \\
\hline B. smooth terms & edf & Ref.df & F-value & p-value \\
Location Smooth & 120.6938 & 315.0000 & 2.0014 & $<0.0001$ \\
Year Smooth & 4.1641 & 19.0000 & 14.2159 & 0.0194 \\
Station Random Effect & 0.3552 & 7.0000 & 0.2239 & 0.2448 \\
\hline
\end{tabular}

Table A3.62: Best productivity model for the House Wren (Troglodytes aedon). The model was a quasi-Poisson GAMM with log link function. Based on AIC/QIC, support for including the noise level as a predictor of productivity was: weak; support for including the standard deviation of noise was: none. 


\begin{tabular}{lrrrr}
\hline A. parametric coefficients & Estimate & Std. Error & t-value & p-value \\
Intercept & -6.1583 & 0.1244 & -49.4988 & $<0.0001$ \\
PC 1 & 0.0993 & 0.0990 & 1.0034 & 0.3157 \\
PC 2 & 0.3390 & 0.1050 & 3.2289 & 0.0013 \\
PC 3 & 0.0610 & 0.1026 & 0.5945 & 0.5522 \\
PC 4 & -0.3261 & 0.0990 & -3.2938 & 0.0010 \\
PC 5 & 0.0850 & 0.1179 & 0.7213 & 0.4708 \\
PC 6 & 0.0681 & 0.1068 & 0.6374 & 0.5239 \\
PC 7 & 0.2232 & 0.1156 & 1.9301 & 0.0537 \\
PC 8 & -0.1306 & 0.1841 & -0.7094 & 0.4781 \\
\hline B. smooth terms & edf & Ref.df & F-value & p-value \\
Location Smooth & 10.8154 & 19.0000 & 2972.3615 & 0.1970 \\
Year Smooth & 3.2325 & 7.0000 & 286.4170 & 0.0001 \\
Station Random Effect & 311.4291 & 390.0000 & 8.3659 & $<0.0001$ \\
\hline
\end{tabular}

Table A3.63: Best abundance model for the House Wren (Troglodytes aedon). The model was a quasi-Poisson GAMM with log link function. Based on AIC/QIC, support for including the noise level as a predictor of abundance was: none; support for including the standard deviation of noise was: none.

\begin{tabular}{lrrrr}
\hline A. parametric coefficients & Estimate & Std. Error & t-value & p-value \\
Intercept & -2.7423 & 0.2196 & -12.4869 & $<0.0001$ \\
Noise Standard Deviation & 0.0490 & 0.1887 & 0.2598 & 0.7950 \\
PC 1 & -0.1292 & 0.0981 & -1.3171 & 0.1880 \\
PC 2 & -0.2683 & 0.1099 & -2.4412 & 0.0147 \\
PC 3 & -0.0924 & 0.2116 & -0.4370 & 0.6622 \\
PC 4 & -0.1370 & 0.1468 & -0.9332 & 0.3508 \\
PC 5 & 0.2497 & 0.1630 & 1.5319 & 0.1257 \\
PC 6 & -0.0749 & 0.1659 & -0.4516 & 0.6516 \\
PC 7 & 0.1195 & 0.1449 & 0.8247 & 0.4096 \\
PC 8 & -0.0430 & 0.1871 & -0.2299 & 0.8182 \\
\hline B. smooth terms & edf & Ref.df & F-value & p-value \\
Location Smooth & 107.6952 & 369.0000 & 0.5919 & $<0.0001$ \\
Year Smooth & 9.8512 & 19.0000 & 18.9718 & $<0.0001$ \\
Station Random Effect & 5.5957 & 7.0000 & 7.8887 & 0.0003 \\
\hline
\end{tabular}

Table A3.64: Best productivity model for the Indigo Bunting (Passerina cyanea). The model was a quasi-Poisson GAMM with log link function. Based on AIC/QIC, support for including the noise level as a predictor of productivity was: none; support for including the standard deviation of noise was: weak. 


\begin{tabular}{lrrrr}
\hline A. parametric coefficients & Estimate & Std. Error & t-value & p-value \\
Intercept & -5.5185 & 0.1761 & -31.3297 & $<0.0001$ \\
Noise Standard Deviation & 0.1192 & 0.1742 & 0.6843 & 0.4938 \\
PC 1 & 0.0187 & 0.0767 & 0.2435 & 0.8076 \\
PC 2 & 0.0157 & 0.0872 & 0.1803 & 0.8570 \\
PC 3 & -0.2282 & 0.1514 & -1.5071 & 0.1319 \\
PC 4 & -0.2115 & 0.1112 & -1.9011 & 0.0574 \\
PC 5 & 0.1016 & 0.1254 & 0.8102 & 0.4179 \\
PC 6 & -0.0829 & 0.1255 & -0.6601 & 0.5092 \\
PC 7 & 0.1133 & 0.1102 & 1.0277 & 0.3042 \\
PC 8 & -0.1573 & 0.1607 & -0.9794 & 0.3275 \\
\hline B. smooth terms & edf & Ref.df & F-value & p-value \\
Location Smooth & 12.8156 & 19.0000 & 1544.5176 & $<0.0001$ \\
Year Smooth & 5.4171 & 7.0000 & 92.1541 & $<0.0001$ \\
Station Random Effect & 286.0567 & 375.0000 & 7.1383 & $<0.0001$ \\
\hline
\end{tabular}

Table A3.65: Best abundance model for the Indigo Bunting (Passerina cyanea). The model was a quasi-Poisson GAMM with log link function. Based on AIC/QIC, support for including the noise level as a predictor of abundance was: none; support for including the standard deviation of noise was: weak.

\begin{tabular}{lrrrr}
\hline A. parametric coefficients & Estimate & Std. Error & t-value & p-value \\
Intercept & -2.3337 & 0.2439 & -9.5674 & $<0.0001$ \\
Noise Standard Deviation & 0.2320 & 0.2097 & 1.1062 & 0.2689 \\
PC 1 & -0.0647 & 0.1247 & -0.5187 & 0.6041 \\
PC 2 & -0.2385 & 0.1379 & -1.7301 & 0.0840 \\
PC 3 & 0.1044 & 0.1086 & 0.9608 & 0.3369 \\
PC 4 & -0.1672 & 0.1370 & -1.2204 & 0.2226 \\
PC 5 & 0.0203 & 0.1211 & 0.1678 & 0.8668 \\
PC 6 & -0.2486 & 0.1264 & -1.9672 & 0.0495 \\
PC 7 & -0.0961 & 0.2321 & -0.4141 & 0.6789 \\
PC 8 & 0.1147 & 0.3511 & 0.3268 & 0.7439 \\
\hline B. smooth terms & edf & Ref.df & F-value & p-value \\
Location Smooth & 87.1417 & 227.0000 & 1.3944 & $<0.0001$ \\
Year Smooth & 1.6605 & 19.0000 & 40.5738 & 0.0028 \\
Station Random Effect & 1.0217 & 7.0000 & 1.0753 & 0.1250 \\
\hline
\end{tabular}

Table A3.66: Best productivity model for the Lazuli Bunting (Passerina amoena). The model was a quasi-Poisson GAMM with log link function. Based on AIC/QIC, support for including the noise level as a predictor of productivity was: none; support for including the standard deviation of noise was: weak. 


\begin{tabular}{lrrrr}
\hline A. parametric coefficients & Estimate & Std. Error & t-value & p-value \\
Intercept & -6.3314 & 0.1365 & -46.3813 & $<0.0001$ \\
PC 1 & -0.0590 & 0.1349 & -0.4373 & 0.6619 \\
PC 2 & 0.0489 & 0.1271 & 0.3845 & 0.7006 \\
PC 3 & 0.3363 & 0.1030 & 3.2660 & 0.0011 \\
PC 4 & -0.3855 & 0.1126 & -3.4232 & 0.0006 \\
PC 5 & -0.2219 & 0.1112 & -1.9961 & 0.0461 \\
PC 6 & 0.0010 & 0.1165 & 0.0087 & 0.9931 \\
PC 7 & -0.0655 & 0.1697 & -0.3862 & 0.6994 \\
PC 8 & -0.0264 & 0.3594 & -0.0734 & 0.9415 \\
\hline B. smooth terms & edf & Ref.df & F-value & p-value \\
Location Smooth & 4.3696 & 19.0000 & 140.3974 & 0.3490 \\
Year Smooth & 5.5901 & 7.0000 & 23.2137 & $<0.0001$ \\
Station Random Effect & 185.1872 & 241.0000 & 6.8078 & $<0.0001$ \\
\hline
\end{tabular}

Table A3.67: Best abundance model for the Lazuli Bunting (Passerina amoena). The model was a quasi-Poisson GAMM with log link function. Based on AIC/QIC, support for including the noise level as a predictor of abundance was: none; support for including the standard deviation of noise was: none.

\begin{tabular}{lrrrr}
\hline A. parametric coefficients & Estimate & Std. Error & t-value & p-value \\
Intercept & -2.2694 & 0.3490 & -6.5023 & $<0.0001$ \\
PC 1 & -0.3451 & 0.3151 & -1.0953 & 0.2743 \\
PC 2 & -0.0407 & 0.2848 & -0.1430 & 0.8864 \\
PC 3 & -0.4802 & 0.3868 & -1.2414 & 0.2154 \\
PC 4 & -0.1933 & 0.4095 & -0.4720 & 0.6373 \\
PC 5 & 0.3802 & 0.5136 & 0.7403 & 0.4597 \\
PC 6 & 0.2687 & 0.4565 & 0.5886 & 0.5566 \\
PC 7 & -0.3132 & 0.3524 & -0.8889 & 0.3747 \\
PC 8 & 1.0459 & 0.8352 & 1.2523 & 0.2114 \\
\hline B. smooth terms & edf & Ref.df & F-value & p-value \\
Location Smooth & 20.0315 & 106.0000 & 0.2732 & 0.0210 \\
Year Smooth & 1.6296 & 19.0000 & 0.4901 & 0.1358 \\
Station Random Effect & 4.3473 & 7.0000 & 3.0040 & 0.0356 \\
\hline
\end{tabular}

Table A3.68: Best productivity model for the Least Flycatcher (Empidonax minimus). The model was a quasi-Poisson GAMM with log link function. Based on AIC/QIC, support for including the noise level as a predictor of productivity was: none; support for including the standard deviation of noise was: none. 


\begin{tabular}{lrrrr}
\hline A. parametric coefficients & Estimate & Std. Error & t-value & p-value \\
Intercept & -2.9064 & 2.8208 & -1.0303 & 0.3031 \\
Noise Level & -0.1091 & 0.0761 & -1.4350 & 0.1516 \\
PC 1 & 0.2488 & 0.2502 & 0.9945 & 0.3202 \\
PC 2 & -0.0537 & 0.1917 & -0.2803 & 0.7793 \\
PC 3 & -0.3773 & 0.2491 & -1.5148 & 0.1302 \\
PC 4 & -0.3562 & 0.2706 & -1.3167 & 0.1883 \\
PC 5 & 0.3116 & 0.3411 & 0.9136 & 0.3612 \\
PC 6 & 0.1126 & 0.2722 & 0.4136 & 0.6793 \\
PC 7 & 0.0645 & 0.2303 & 0.2803 & 0.7793 \\
PC 8 & -0.1474 & 0.3758 & -0.3923 & 0.6949 \\
\hline B. smooth terms & edf & Ref.df & F-value & p-value \\
Location Smooth & 9.5493 & 19.0000 & 150.9727 & 0.0008 \\
Year Smooth & 5.0032 & 7.0000 & 5.2464 & 0.0826 \\
Station Random Effect & 69.0704 & 110.0000 & 3.0388 & $<0.0001$ \\
\hline
\end{tabular}

Table A3.69: Best abundance model for the Least Flycatcher (Empidonax minimus). The model was a quasi-Poisson GAMM with log link function. Based on AIC/QIC, support for including the noise level as a predictor of abundance was: weak; support for including the standard deviation of noise was: none.

\begin{tabular}{lrrrr}
\hline A. parametric coefficients & Estimate & Std. Error & t-value & p-value \\
Intercept & 2.0993 & 1.7615 & 1.1918 & 0.2337 \\
Noise Level & -0.1194 & 0.0523 & -2.2848 & 0.0226 \\
PC 1 & 0.0441 & 0.2126 & 0.2074 & 0.8358 \\
PC 2 & -0.5771 & 0.3461 & -1.6671 & 0.0959 \\
PC 3 & 0.3960 & 0.3094 & 1.2799 & 0.2010 \\
PC 4 & -0.6791 & 0.5604 & -1.2118 & 0.2260 \\
PC 5 & 0.1924 & 0.3465 & 0.5552 & 0.5789 \\
PC 6 & -0.2579 & 0.3171 & -0.8132 & 0.4164 \\
PC 7 & -0.5691 & 0.8415 & -0.6762 & 0.4991 \\
PC 8 & -0.2408 & 0.4057 & -0.5936 & 0.5530 \\
\hline B. smooth terms & edf & Ref.df & F-value & p-value \\
Location Smooth & 23.9046 & 108.0000 & 0.8536 & $<0.0001$ \\
Year Smooth & 1.8226 & 19.0000 & 4.2375 & 0.0080 \\
Station Random Effect & 1.7810 & 7.0000 & 0.8392 & 0.0747 \\
\hline
\end{tabular}

Table A3.70: Best productivity model for the Lincoln's Sparrow (Melospiza lincolnii). The model was a quasi-Poisson GAMM with log link function. Based on AIC/QIC, support for including the noise level as a predictor of productivity was: strong; support for including the standard deviation of noise was: none. 


\begin{tabular}{lrrrr}
\hline A. parametric coefficients & Estimate & Std. Error & t-value & p-value \\
Intercept & -3.9772 & 4.1559 & -0.9570 & 0.3387 \\
Noise Level & -0.0577 & 0.1210 & -0.4766 & 0.6337 \\
PC 1 & -0.1414 & 0.4788 & -0.2953 & 0.7678 \\
PC 2 & -0.6071 & 0.3437 & -1.7663 & 0.0776 \\
PC 3 & -0.4235 & 0.2472 & -1.7131 & 0.0869 \\
PC 4 & -0.3325 & 0.5196 & -0.6399 & 0.5224 \\
PC 5 & -0.2118 & 0.6579 & -0.3219 & 0.7476 \\
PC 6 & -0.1298 & 0.4122 & -0.3148 & 0.7530 \\
PC 7 & -0.2470 & 0.5941 & -0.4159 & 0.6776 \\
PC 8 & 0.9012 & 0.9793 & 0.9203 & 0.3576 \\
\hline B. smooth terms & edf & Ref.df & F-value & p-value \\
Location Smooth & 11.5120 & 19.0000 & 22262.5235 & 0.0008 \\
Year Smooth & 4.4842 & 7.0000 & 10.3767 & 0.2003 \\
Station Random Effect & 96.0500 & 125.0000 & 13.6719 & $<0.0001$ \\
\hline
\end{tabular}

Table A3.71: Best abundance model for the Lincoln's Sparrow (Melospiza lincolnii). The model was a quasi-Poisson GAMM with log link function. Based on AIC/QIC, support for including the noise level as a predictor of abundance was: weak; support for including the standard deviation of noise was: none.

\begin{tabular}{lrrrr}
\hline A. parametric coefficients & Estimate & Std. Error & t-value & p-value \\
Intercept & -1.6233 & 0.8939 & -1.8159 & 0.0708 \\
PC 1 & -1.6246 & 0.7460 & -2.1778 & 0.0306 \\
PC 2 & 1.2203 & 0.3738 & 3.2643 & 0.0013 \\
PC 3 & 1.4342 & 0.7702 & 1.8622 & 0.0640 \\
PC 4 & -0.4467 & 0.4311 & -1.0361 & 0.3014 \\
PC 5 & 0.6230 & 0.4799 & 1.2983 & 0.1956 \\
PC 6 & 0.6847 & 1.0861 & 0.6304 & 0.5291 \\
PC 7 & 0.2566 & 0.3685 & 0.6964 & 0.4869 \\
PC 8 & 0.1637 & 1.5808 & 0.1036 & 0.9176 \\
\hline B. smooth terms & edf & Ref.df & F-value & p-value \\
Location Smooth & 4.0766 & 54.0000 & 0.1329 & 0.0458 \\
Year Smooth & 1.7566 & 19.0000 & 0.7422 & 0.0062 \\
Station Random Effect & 3.8855 & 7.0000 & 1.4187 & 0.0590 \\
\hline
\end{tabular}

Table A3.72: Best productivity model for the Magnolia Warbler (Setophaga magnolia). The model was a quasi-Poisson GAMM with log link function. Based on AIC/QIC, support for including the noise level as a predictor of productivity was: none; support for including the standard deviation of noise was: none. 


\begin{tabular}{lrrrr}
\hline A. parametric coefficients & Estimate & Std. Error & t-value & p-value \\
Intercept & -2.6439 & 3.7065 & -0.7133 & 0.4761 \\
Noise Level & -0.1013 & 0.0948 & -1.0688 & 0.2858 \\
PC 1 & -0.2503 & 0.2888 & -0.8668 & 0.3866 \\
PC 2 & 0.0027 & 0.2894 & 0.0094 & 0.9925 \\
PC 3 & -0.5147 & 0.7755 & -0.6638 & 0.5072 \\
PC 4 & 0.5345 & 0.4447 & 1.2019 & 0.2301 \\
PC 5 & -0.1516 & 0.5564 & -0.2725 & 0.7853 \\
PC 6 & -0.7959 & 1.1051 & -0.7201 & 0.4718 \\
PC 7 & -0.0731 & 0.3160 & -0.2312 & 0.8173 \\
PC 8 & 0.1135 & 0.3072 & 0.3695 & 0.7120 \\
\hline B. smooth terms & edf & Ref.df & F-value & p-value \\
Location Smooth & 1.7185 & 19.0000 & 46.4217 & 0.0031 \\
Year Smooth & 3.4054 & 7.0000 & 18.7662 & 0.0313 \\
Station Random Effect & 38.2189 & 54.0000 & 5.3567 & $<0.0001$ \\
\hline
\end{tabular}

Table A3.73: Best abundance model for the Magnolia Warbler (Setophaga magnolia). The model was a quasi-Poisson GAMM with log link function. Based on AIC/QIC, support for including the noise level as a predictor of abundance was: weak; support for including the standard deviation of noise was: none.

\begin{tabular}{lrrrr}
\hline A. parametric coefficients & Estimate & Std. Error & t-value & p-value \\
Intercept & -3.2028 & 1.2425 & -2.5778 & 0.0100 \\
Noise Level & 0.0464 & 0.0355 & 1.3076 & 0.1912 \\
PC 1 & -0.3785 & 0.2541 & -1.4898 & 0.1365 \\
PC 2 & 0.2489 & 0.1590 & 1.5659 & 0.1176 \\
PC 3 & -0.0588 & 0.0908 & -0.6478 & 0.5172 \\
PC 4 & -0.0616 & 0.1297 & -0.4749 & 0.6349 \\
PC 5 & 0.1151 & 0.1410 & 0.8167 & 0.4142 \\
PC 6 & 0.2146 & 0.1396 & 1.5379 & 0.1242 \\
PC 7 & 0.0982 & 0.1634 & 0.6013 & 0.5477 \\
PC 8 & -0.4226 & 0.4379 & -0.9650 & 0.3347 \\
\hline B. smooth terms & edf & Ref.df & F-value & p-value \\
Location Smooth & 95.4481 & 254.0000 & 1.9754 & $<0.0001$ \\
Year Smooth & 11.2501 & 19.0000 & 132.8603 & 0.0250 \\
Station Random Effect & 0.9349 & 7.0000 & 4.0141 & 0.0003 \\
\hline
\end{tabular}

Table A3.74: Best productivity model for the MacGillivray's Warbler (Geothlypis tolmiei). The model was a quasi-Poisson GAMM with log link function. Based on AIC/QIC, support for including the noise level as a predictor of productivity was: strong; support for including the standard deviation of noise was: none. 


\begin{tabular}{lrrrr}
\hline A. parametric coefficients & Estimate & Std. Error & t-value & p-value \\
Intercept & -5.5341 & 0.2119 & -26.1164 & $<0.0001$ \\
Noise Standard Deviation & 0.1247 & 0.1764 & 0.7068 & 0.4797 \\
PC 1 & -0.7184 & 0.1583 & -4.5397 & $<0.0001$ \\
PC 2 & -0.3905 & 0.1243 & -3.1409 & 0.0017 \\
PC 3 & -0.0336 & 0.0943 & -0.3564 & 0.7215 \\
PC 4 & -0.0484 & 0.1219 & -0.3973 & 0.6912 \\
PC 5 & -0.0852 & 0.1303 & -0.6539 & 0.5132 \\
PC 6 & -0.1409 & 0.1398 & -1.0080 & 0.3135 \\
PC 7 & 0.0196 & 0.1689 & 0.1160 & 0.9077 \\
PC 8 & -0.4244 & 0.3861 & -1.0993 & 0.2718 \\
\hline B. smooth terms & edf & Ref.df & F-value & p-value \\
Location Smooth & 13.6829 & 19.0000 & 5475.7041 & 0.1054 \\
Year Smooth & 4.1927 & 7.0000 & 158.7050 & $<0.0001$ \\
Station Random Effect & 199.3198 & 257.0000 & 15.4350 & $<0.0001$ \\
\hline
\end{tabular}

Table A3.75: Best abundance model for the MacGillivray's Warbler (Geothlypis tolmiei). The model was a quasi-Poisson GAMM with log link function. Based on AIC/QIC, support for including the noise level as a predictor of abundance was: none; support for including the standard deviation of noise was: weak.

\begin{tabular}{lrrrr}
\hline A. parametric coefficients & Estimate & Std. Error & t-value & p-value \\
Intercept & -0.7497 & 0.2831 & -2.6483 & 0.0083 \\
PC 1 & 0.3468 & 0.1514 & 2.2899 & 0.0223 \\
PC 2 & -0.3001 & 0.2130 & -1.4091 & 0.1592 \\
PC 3 & 0.2052 & 0.1488 & 1.3791 & 0.1683 \\
PC 4 & -0.0665 & 0.5083 & -0.1309 & 0.8959 \\
PC 5 & 1.1355 & 0.5224 & 2.1735 & 0.0301 \\
PC 6 & -0.4434 & 0.2977 & -1.4897 & 0.1367 \\
PC 7 & 0.1979 & 0.4598 & 0.4304 & 0.6670 \\
PC 8 & 0.2352 & 0.4055 & 0.5800 & 0.5621 \\
\hline B. smooth terms & edf & Ref.df & F-value & p-value \\
Location Smooth & 17.6297 & 114.0000 & 0.2361 & 0.0157 \\
Year Smooth & 1.8575 & 19.0000 & 1.6164 & 0.0006 \\
Station Random Effect & 4.6064 & 7.0000 & 1.6495 & 0.0492 \\
\hline
\end{tabular}

Table A3.76: Best productivity model for the Mountain Chickadee (Poecile gambeli). The model was a quasi-Poisson GAMM with log link function. Based on AIC/QIC, support for including the noise level as a predictor of productivity was: none; support for including the standard deviation of noise was: none. 


\begin{tabular}{lrrrr}
\hline A. parametric coefficients & Estimate & Std. Error & t-value & p-value \\
Intercept & -6.6374 & 0.3523 & -18.8427 & $<0.0001$ \\
Noise Standard Deviation & 0.2769 & 0.2246 & 1.2332 & 0.2177 \\
PC 1 & -0.2667 & 0.2993 & -0.8911 & 0.3730 \\
PC 2 & -0.3780 & 0.2282 & -1.6566 & 0.0978 \\
PC 3 & -0.0223 & 0.1657 & -0.1344 & 0.8931 \\
PC 4 & -0.7567 & 0.4173 & -1.8130 & 0.0701 \\
PC 5 & 1.4532 & 0.4285 & 3.3914 & 0.0007 \\
PC 6 & -0.2529 & 0.2700 & -0.9365 & 0.3492 \\
PC 7 & 0.8012 & 0.3800 & 2.1085 & 0.0352 \\
PC 8 & -0.7984 & 0.7932 & -1.0065 & 0.3144 \\
\hline B. smooth terms & edf & Ref.df & F-value & p-value \\
Location Smooth & 13.2785 & 19.0000 & 307.3745 & 0.0032 \\
Year Smooth & 5.0776 & 7.0000 & 8.0810 & 0.0001 \\
Station Random Effect & 80.1339 & 124.0000 & 4.4030 & $<0.0001$ \\
\hline
\end{tabular}

Table A3.77: Best abundance model for the Mountain Chickadee (Poecile gambeli). The model was a quasi-Poisson GAMM with log link function. Based on AIC/QIC, support for including the noise level as a predictor of abundance was: none; support for including the standard deviation of noise was: weak.

\begin{tabular}{lrrrr}
\hline A. parametric coefficients & Estimate & Std. Error & t-value & p-value \\
Intercept & -3.5032 & 0.6954 & -5.0379 & $<0.0001$ \\
PC 1 & -0.0045 & 0.3807 & -0.0117 & 0.9907 \\
PC 2 & -0.2250 & 0.4977 & -0.4520 & 0.6517 \\
PC 3 & -0.4373 & 0.5352 & -0.8171 & 0.4147 \\
PC 4 & 0.3907 & 0.3887 & 1.0053 & 0.3158 \\
PC 5 & -0.5794 & 0.5082 & -1.1402 & 0.2554 \\
PC 6 & -0.2315 & 0.6992 & -0.3311 & 0.7409 \\
PC 7 & 0.5587 & 0.4443 & 1.2577 & 0.2098 \\
PC 8 & -0.1948 & 0.9280 & -0.2099 & 0.8339 \\
\hline B. smooth terms & edf & Ref.df & F-value & p-value \\
Location Smooth & 68.1619 & 161.0000 & 0.4253 & 0.4342 \\
Year Smooth & 1.4339 & 19.0000 & 10.6970 & 0.4666 \\
Station Random Effect & 3.2982 & 7.0000 & 41.3259 & 0.2773 \\
\hline
\end{tabular}

Table A3.78: Best productivity model for the Mourning Dove (Zenaida macroura). The model was a quasi-Poisson GAMM with log link function. Based on AIC/QIC, support for including the noise level as a predictor of productivity was: none; support for including the standard deviation of noise was: none. 


\begin{tabular}{lrrrr}
\hline A. parametric coefficients & Estimate & Std. Error & t-value & p-value \\
Intercept & -8.1137 & 0.1307 & -62.1015 & $<0.0001$ \\
PC 1 & 0.2782 & 0.0776 & 3.5825 & 0.0003 \\
PC 2 & 0.0808 & 0.0919 & 0.8789 & 0.3796 \\
PC 3 & 0.1124 & 0.0802 & 1.4003 & 0.1616 \\
PC 4 & -0.0049 & 0.0781 & -0.0633 & 0.9496 \\
PC 5 & -0.0553 & 0.0896 & -0.6174 & 0.5371 \\
PC 6 & -0.0596 & 0.0967 & -0.6160 & 0.5380 \\
PC 7 & -0.0044 & 0.0980 & -0.0451 & 0.9641 \\
PC 8 & -0.1109 & 0.1559 & -0.7109 & 0.4772 \\
\hline B. smooth terms & edf & Ref.df & F-value & p-value \\
Location Smooth & 0.8477 & 19.0000 & 0.3361 & 0.3323 \\
Year Smooth & 4.2480 & 7.0000 & 4.3005 & 0.0981 \\
Station Random Effect & 117.8810 & 208.0000 & 1.4896 & $<0.0001$ \\
\hline
\end{tabular}

Table A3.79: Best abundance model for the Mourning Dove (Zenaida macroura). The model was a quasi-Poisson GAMM with log link function. Based on AIC/QIC, support for including the noise level as a predictor of abundance was: none; support for including the standard deviation of noise was: none. 


\begin{tabular}{lrrrr}
\hline A. parametric coefficients & Estimate & Std. Error & t-value & p-value \\
Intercept & 58.6599 & 22.1184 & 2.6521 & 0.0088 \\
Noise Level & -1.8245 & 0.6696 & -2.7248 & 0.0072 \\
Noise Standard Deviation & 4.5938 & 1.6340 & 2.8114 & 0.0056 \\
PC 1 & 3.1338 & 1.5265 & 2.0530 & 0.0418 \\
PC 2 & 0.2659 & 0.8654 & 0.3072 & 0.7591 \\
PC 3 & -1.3325 & 3.0434 & -0.4378 & 0.6621 \\
PC 4 & -1.7530 & 1.1902 & -1.4729 & 0.1428 \\
PC 5 & 2.0334 & 1.1592 & 1.7542 & 0.0814 \\
PC 6 & 4.7180 & 2.9664 & 1.5905 & 0.1138 \\
PC 7 & -1.7542 & 1.3512 & -1.2982 & 0.1962 \\
PC 8 & 5.1242 & 2.2152 & 2.3132 & 0.0220 \\
\hline B. smooth terms & edf & Ref.df & F-value & p-value \\
Location Smooth & 0.0001 & 43.0000 & 0.0000 & 0.2885 \\
Year Smooth & 0.0000 & 19.0000 & 0.0000 & 1.0000 \\
Station Random Effect & 3.3687 & 7.0000 & 1.7089 & 0.0072 \\
\hline
\end{tabular}

Table A3.80: Best productivity model for the Myrtle Warbler (Setophaga coronata coronata). The model was a quasi-Poisson GAMM with log link function. Based on AIC/QIC, support for including the noise level as a predictor of productivity was: strong; support for including the standard deviation of noise was: strong. 


\begin{tabular}{lrrrr}
\hline A. parametric coefficients & Estimate & Std. Error & t-value & p-value \\
Intercept & 10.1253 & 4.2203 & 2.3992 & 0.0170 \\
Noise Level & -0.4617 & 0.1148 & -4.0227 & 0.0001 \\
PC 1 & 0.3251 & 0.3357 & 0.9683 & 0.3336 \\
PC 2 & -0.2815 & 0.3208 & -0.8775 & 0.3808 \\
PC 3 & -0.6062 & 0.7308 & -0.8296 & 0.4073 \\
PC 4 & -0.0040 & 0.4376 & -0.0092 & 0.9927 \\
PC 5 & 0.2805 & 0.5371 & 0.5221 & 0.6019 \\
PC 6 & 0.9188 & 0.9607 & 0.9564 & 0.3395 \\
PC 7 & -0.2699 & 0.2508 & -1.0763 & 0.2826 \\
PC 8 & -0.0495 & 0.6385 & -0.0776 & 0.9382 \\
\hline B. smooth terms & edf & Ref.df & F-value & p-value \\
Location Smooth & 4.3065 & 19.0000 & 6.3119 & 0.3729 \\
Year Smooth & 0.9376 & 7.0000 & 6.5967 & 0.0002 \\
Station Random Effect & 28.5464 & 46.0000 & 2.8940 & $<0.0001$ \\
\hline
\end{tabular}

Table A3.81: Best abundance model for the Myrtle Warbler (Setophaga coronata coronata). The model was a quasi-Poisson GAMM with log link function. Based on AIC/QIC, support for including the noise level as a predictor of abundance was: strong; support for including the standard deviation of noise was: none.

\begin{tabular}{lrrrr}
\hline A. parametric coefficients & Estimate & Std. Error & t-value & p-value \\
Intercept & 4.6831 & 3.3955 & 1.3792 & 0.1683 \\
Noise Level & -0.1643 & 0.0955 & -1.7198 & 0.0860 \\
Noise Standard Deviation & 0.2902 & 0.3122 & 0.9294 & 0.3530 \\
PC 1 & 0.1893 & 0.2813 & 0.6729 & 0.5013 \\
PC 2 & -0.2445 & 0.2194 & -1.1145 & 0.2655 \\
PC 3 & -0.0634 & 0.2139 & -0.2964 & 0.7670 \\
PC 4 & -0.2941 & 0.2559 & -1.1492 & 0.2509 \\
PC 5 & 0.4100 & 0.3955 & 1.0367 & 0.3003 \\
PC 6 & 0.0854 & 0.2866 & 0.2979 & 0.7659 \\
PC 7 & 0.0699 & 0.3328 & 0.2102 & 0.8336 \\
PC 8 & -1.0212 & 0.5968 & -1.7110 & 0.0876 \\
\hline B. smooth terms & edf & Ref.df & F-value & p-value \\
Location Smooth & 56.0398 & 127.0000 & 4.1218 & $<0.0001$ \\
Year Smooth & 0.0003 & 19.0000 & 0.0000 & 0.9962 \\
Station Random Effect & 3.7514 & 7.0000 & 6.6571 & 0.0097 \\
\hline
\end{tabular}

Table A3.82: Best productivity model for the Nashville Warbler (Oreothlypis ruficapilla). The model was a quasi-Poisson GAMM with log link function. Based on AIC/QIC, support for including the noise level as a predictor of productivity was: strong; support for including the standard deviation of noise was: weak. 


\begin{tabular}{lrrrr}
\hline A. parametric coefficients & Estimate & Std. Error & t-value & p-value \\
Intercept & -3.9409 & 2.7016 & -1.4587 & 0.1449 \\
Noise Level & -0.0601 & 0.0749 & -0.8026 & 0.4224 \\
PC 1 & 0.0751 & 0.2127 & 0.3532 & 0.7240 \\
PC 2 & -0.3242 & 0.1568 & -2.0673 & 0.0389 \\
PC 3 & 0.1844 & 0.1814 & 1.0167 & 0.3095 \\
PC 4 & 0.0560 & 0.1542 & 0.3630 & 0.7167 \\
PC 5 & 0.4031 & 0.1956 & 2.0602 & 0.0396 \\
PC 6 & -0.1514 & 0.2163 & -0.6997 & 0.4843 \\
PC 7 & 0.0146 & 0.1950 & 0.0750 & 0.9403 \\
PC 8 & 0.6066 & 0.4854 & 1.2497 & 0.2117 \\
\hline B. smooth terms & edf & Ref.df & F-value & p-value \\
Location Smooth & 8.1910 & 19.0000 & 308.8057 & 0.0502 \\
Year Smooth & 4.8342 & 7.0000 & 10.8257 & 0.0020 \\
Station Random Effect & 100.5714 & 143.0000 & 5.1745 & $<0.0001$ \\
\hline
\end{tabular}

Table A3.83: Best abundance model for the Nashville Warbler (Oreothlypis ruficapilla). The model was a quasi-Poisson GAMM with log link function. Based on AIC/QIC, support for including the noise level as a predictor of abundance was: weak; support for including the standard deviation of noise was: none.

\begin{tabular}{lrrrr}
\hline A. parametric coefficients & Estimate & Std. Error & t-value & p-value \\
Intercept & -2.3137 & 0.6376 & -3.6287 & 0.0003 \\
Noise Level & 0.0260 & 0.0159 & 1.6296 & 0.1033 \\
PC 1 & -0.0176 & 0.0476 & -0.3689 & 0.7123 \\
PC 2 & 0.0353 & 0.0426 & 0.8294 & 0.4070 \\
PC 3 & 0.0641 & 0.0619 & 1.0356 & 0.3005 \\
PC 4 & -0.0539 & 0.0573 & -0.9408 & 0.3469 \\
PC 5 & 0.0196 & 0.0647 & 0.3031 & 0.7619 \\
PC 6 & -0.0177 & 0.0565 & -0.3125 & 0.7547 \\
PC 7 & 0.0649 & 0.0565 & 1.1498 & 0.2503 \\
PC 8 & -0.0636 & 0.0616 & -1.0326 & 0.3019 \\
\hline B. smooth terms & edf & Ref.df & F-value & p-value \\
Location Smooth & 188.5597 & 449.0000 & 1.6840 & $<0.0001$ \\
Year Smooth & 7.4274 & 19.0000 & 24.1008 & 0.0002 \\
Station Random Effect & 5.7339 & 7.0000 & 20.7779 & $<0.0001$ \\
\hline
\end{tabular}

Table A3.84: Best productivity model for the Northern Cardinal (Cardinalis cardinalis). The model was a quasi-Poisson GAMM with log link function. Based on AIC/QIC, support for including the noise level as a predictor of productivity was: strong; support for including the standard deviation of noise was: none. 


\begin{tabular}{lrrrr}
\hline A. parametric coefficients & Estimate & Std. Error & t-value & p-value \\
Intercept & -4.6378 & 0.0929 & -49.9066 & $<0.0001$ \\
Noise Standard Deviation & -0.0708 & 0.0981 & -0.7220 & 0.4704 \\
PC 1 & 0.2483 & 0.0352 & 7.0476 & $<0.0001$ \\
PC 2 & 0.0254 & 0.0433 & 0.5878 & 0.5567 \\
PC 3 & -0.1094 & 0.0694 & -1.5773 & 0.1148 \\
PC 4 & -0.0176 & 0.0562 & -0.3136 & 0.7538 \\
PC 5 & -0.0847 & 0.0632 & -1.3416 & 0.1798 \\
PC 6 & -0.0860 & 0.0593 & -1.4501 & 0.1471 \\
PC 7 & 0.1073 & 0.0548 & 1.9582 & 0.0503 \\
PC 8 & -0.1400 & 0.0579 & -2.4186 & 0.0156 \\
\hline B. smooth terms & edf & Ref.df & F-value & p-value \\
Location Smooth & 13.1513 & 19.0000 & 4901.6289 & $<0.0001$ \\
Year Smooth & 4.3820 & 7.0000 & 37.0642 & 0.0009 \\
Station Random Effect & 362.3280 & 450.0000 & 8.0568 & $<0.0001$ \\
\hline
\end{tabular}

Table A3.85: Best abundance model for the Northern Cardinal (Cardinalis cardinalis). The model was a quasi-Poisson GAMM with log link function. Based on AIC/QIC, support for including the noise level as a predictor of abundance was: none; support for including the standard deviation of noise was: weak.

\begin{tabular}{lrrrr}
\hline A. parametric coefficients & Estimate & Std. Error & t-value & p-value \\
Intercept & -1.0994 & 0.2061 & -5.3343 & $<0.0001$ \\
Noise Standard Deviation & 0.0473 & 0.1900 & 0.2490 & 0.8034 \\
PC 1 & -0.1443 & 0.0986 & -1.4627 & 0.1439 \\
PC 2 & 0.0650 & 0.1209 & 0.5376 & 0.5910 \\
PC 3 & -0.0943 & 0.1172 & -0.8049 & 0.4211 \\
PC 4 & -0.1603 & 0.0985 & -1.6278 & 0.1039 \\
PC 5 & -0.1383 & 0.1315 & -1.0513 & 0.2934 \\
PC 6 & 0.1222 & 0.1371 & 0.8912 & 0.3730 \\
PC 7 & 0.0204 & 0.1348 & 0.1514 & 0.8797 \\
PC 8 & -0.2018 & 0.2426 & -0.8316 & 0.4059 \\
\hline B. smooth terms & edf & Ref.df & F-value & p-value \\
Location Smooth & 82.7266 & 189.0000 & 2.5531 & $<0.0001$ \\
Year Smooth & 10.1667 & 19.0000 & 72.5568 & 0.0182 \\
Station Random Effect & 3.9339 & 7.0000 & 7.6642 & 0.0356 \\
\hline
\end{tabular}

Table A3.86: Best productivity model for the Orange-crowned Warbler (Oreothlypis celata). The model was a quasi-Poisson GAMM with log link function. Based on AIC/QIC, support for including the noise level as a predictor of productivity was: none; support for including the standard deviation of noise was: weak. 


\begin{tabular}{lrrrr}
\hline A. parametric coefficients & Estimate & Std. Error & t-value & p-value \\
Intercept & -8.8759 & 1.1066 & -8.0208 & $<0.0001$ \\
Noise Level & 0.0872 & 0.0306 & 2.8494 & 0.0044 \\
Noise Standard Deviation & -0.1001 & 0.2115 & -0.4732 & 0.6361 \\
PC 1 & -0.1872 & 0.1283 & -1.4594 & 0.1446 \\
PC 2 & 0.0597 & 0.1234 & 0.4842 & 0.6283 \\
PC 3 & 0.0338 & 0.1077 & 0.3140 & 0.7536 \\
PC 4 & 0.1134 & 0.1087 & 1.0433 & 0.2969 \\
PC 5 & 0.0675 & 0.1389 & 0.4858 & 0.6272 \\
PC 6 & -0.1052 & 0.1315 & -0.8002 & 0.4237 \\
PC 7 & -0.0294 & 0.1599 & -0.1842 & 0.8539 \\
PC 8 & -0.2225 & 0.2838 & -0.7841 & 0.4331 \\
\hline B. smooth terms & edf & Ref.df & F-value & p-value \\
Location Smooth & 1.3608 & 19.0000 & 110.1914 & 0.0617 \\
Year Smooth & 5.1321 & 7.0000 & 84.1395 & $<0.0001$ \\
Station Random Effect & 164.7677 & 203.0000 & 9.9069 & $<0.0001$ \\
\hline
\end{tabular}

Table A3.87: Best abundance model for the Orange-crowned Warbler (Oreothlypis celata). The model was a quasi-Poisson GAMM with log link function. Based on AIC/QIC, support for including the noise level as a predictor of abundance was: weak; support for including the standard deviation of noise was: weak.

\begin{tabular}{lrrrr}
\hline A. parametric coefficients & Estimate & Std. Error & t-value & p-value \\
Intercept & -1.8929 & 0.2746 & -6.8924 & $<0.0001$ \\
Noise Standard Deviation & 0.2657 & 0.1572 & 1.6903 & 0.0912 \\
PC 1 & -0.1105 & 0.0752 & -1.4691 & 0.1420 \\
PC 2 & -0.1721 & 0.0952 & -1.8066 & 0.0710 \\
PC 3 & -0.4388 & 0.2800 & -1.5670 & 0.1173 \\
PC 4 & 0.0398 & 0.1302 & 0.3056 & 0.7599 \\
PC 5 & 0.0367 & 0.1589 & 0.2310 & 0.8173 \\
PC 6 & -0.2429 & 0.3023 & -0.8034 & 0.4218 \\
PC 7 & -0.0438 & 0.1314 & -0.3337 & 0.7387 \\
PC 8 & 0.1275 & 0.1286 & 0.9909 & 0.3219 \\
\hline B. smooth terms & edf & Ref.df & F-value & p-value \\
Location Smooth & 130.6412 & 318.0000 & 1.6946 & $<0.0001$ \\
Year Smooth & 3.2576 & 19.0000 & 11.4096 & 0.0175 \\
Station Random Effect & 3.2032 & 7.0000 & 15.5396 & $<0.0001$ \\
\hline
\end{tabular}

Table A3.88: Best productivity model for the Ovenbird (Seiurus aurocapilla). The model was a quasi-Poisson GAMM with log link function. Based on AIC/QIC, support for including the noise level as a predictor of productivity was: none; support for including the standard deviation of noise was: strong. 


\begin{tabular}{lrrrr}
\hline A. parametric coefficients & Estimate & Std. Error & t-value & p-value \\
Intercept & -2.8779 & 1.2203 & -2.3583 & 0.0184 \\
Noise Level & -0.0709 & 0.0300 & -2.3650 & 0.0181 \\
Noise Standard Deviation & 0.1550 & 0.1649 & 0.9400 & 0.3473 \\
PC 1 & -0.1765 & 0.0938 & -1.8816 & 0.0600 \\
PC 2 & -0.2940 & 0.1016 & -2.8942 & 0.0038 \\
PC 3 & 0.0191 & 0.2734 & 0.0697 & 0.9444 \\
PC 4 & -0.0559 & 0.0977 & -0.5727 & 0.5669 \\
PC 5 & 0.1616 & 0.1140 & 1.4179 & 0.1564 \\
PC 6 & 0.0947 & 0.2217 & 0.4272 & 0.6692 \\
PC 7 & -0.1346 & 0.1187 & -1.1344 & 0.2567 \\
PC 8 & -0.0431 & 0.1033 & -0.4166 & 0.6770 \\
\hline B. smooth terms & edf & Ref.df & F-value & p-value \\
Location Smooth & 15.1305 & 19.0000 & 2766.3287 & $<0.0001$ \\
Year Smooth & 5.0687 & 7.0000 & 47.8087 & 0.0001 \\
Station Random Effect & 251.0550 & 329.0000 & 7.1589 & $<0.0001$ \\
\hline
\end{tabular}

Table A3.89: Best abundance model for the Ovenbird (Seiurus aurocapilla). The model was a quasi-Poisson GAMM with log link function. Based on AIC/QIC, support for including the noise level as a predictor of abundance was: strong; support for including the standard deviation of noise was: weak.

\begin{tabular}{lrrrr}
\hline A. parametric coefficients & Estimate & Std. Error & t-value & p-value \\
Intercept & 1.2309 & 3.3499 & 0.3674 & 0.7134 \\
Noise Level & -0.0825 & 0.0969 & -0.8511 & 0.3950 \\
PC 1 & 0.3732 & 0.4021 & 0.9283 & 0.3535 \\
PC 2 & 0.0992 & 0.2771 & 0.3581 & 0.7203 \\
PC 3 & 0.1594 & 0.2236 & 0.7131 & 0.4760 \\
PC 4 & 0.1487 & 0.3900 & 0.3811 & 0.7032 \\
PC 5 & 0.2507 & 0.3618 & 0.6930 & 0.4885 \\
PC 6 & -0.1172 & 0.2556 & -0.4583 & 0.6469 \\
PC 7 & 0.0078 & 0.3063 & 0.0256 & 0.9796 \\
PC 8 & -0.1937 & 0.7763 & -0.2495 & 0.8030 \\
\hline B. smooth terms & edf & Ref.df & F-value & p-value \\
Location Smooth & 50.8367 & 157.0000 & 0.8125 & $<0.0001$ \\
Year Smooth & 5.0387 & 19.0000 & 4.7697 & 0.1010 \\
Station Random Effect & 6.3952 & 7.0000 & 12.3406 & 0.0006 \\
\hline
\end{tabular}

Table A3.90: Best productivity model for the Pine Siskin (Spinus pinus). The model was a quasi-Poisson GAMM with log link function. Based on AIC/QIC, support for including the noise level as a predictor of productivity was: weak; support for including the standard deviation of noise was: none. 


\begin{tabular}{lrrrr}
\hline A. parametric coefficients & Estimate & Std. Error & t-value & p-value \\
Intercept & -5.9610 & 0.2260 & -26.3752 & $<0.0001$ \\
PC 1 & 0.6357 & 0.3092 & 2.0559 & 0.0399 \\
PC 2 & -0.7055 & 0.2219 & -3.1791 & 0.0015 \\
PC 3 & 0.2774 & 0.1604 & 1.7290 & 0.0840 \\
PC 4 & 0.7665 & 0.2564 & 2.9900 & 0.0028 \\
PC 5 & 0.4265 & 0.2354 & 1.8115 & 0.0702 \\
PC 6 & -0.3417 & 0.1940 & -1.7616 & 0.0783 \\
PC 7 & -0.4206 & 0.2511 & -1.6749 & 0.0941 \\
PC 8 & 1.7618 & 0.6102 & 2.8875 & 0.0039 \\
\hline B. smooth terms & edf & Ref.df & F-value & p-value \\
Location Smooth & 9.9008 & 19.0000 & 126.4948 & 0.1462 \\
Year Smooth & 5.7352 & 7.0000 & 19.3483 & $<0.0001$ \\
Station Random Effect & 112.4495 & 166.0000 & 3.6217 & $<0.0001$ \\
\hline
\end{tabular}

Table A3.91: Best abundance model for the Pine Siskin (Spinus pinus). The model was a quasi-Poisson GAMM with log link function. Based on AIC/QIC, support for including the noise level as a predictor of abundance was: none; support for including the standard deviation of noise was: none.

\begin{tabular}{lrrrr}
\hline A. parametric coefficients & Estimate & Std. Error & t-value & p-value \\
Intercept & -1.3606 & 0.3525 & -3.8602 & 0.0001 \\
Noise Standard Deviation & 0.2423 & 0.2232 & 1.0854 & 0.2781 \\
PC 1 & -0.1990 & 0.2932 & -0.6788 & 0.4975 \\
PC 2 & -0.1906 & 0.2231 & -0.8542 & 0.3933 \\
PC 3 & 0.0500 & 0.1619 & 0.3085 & 0.7578 \\
PC 4 & 0.3468 & 0.3174 & 1.0929 & 0.2748 \\
PC 5 & 0.1341 & 0.3487 & 0.3845 & 0.7007 \\
PC 6 & -0.0406 & 0.2275 & -0.1784 & 0.8585 \\
PC 7 & 0.1497 & 0.2339 & 0.6400 & 0.5224 \\
PC 8 & -0.2806 & 0.5404 & -0.5192 & 0.6038 \\
\hline B. smooth terms & edf & Ref.df & F-value & p-value \\
Location Smooth & 52.6359 & 180.0000 & 0.6721 & $<0.0001$ \\
Year Smooth & 4.8422 & 19.0000 & 2.6268 & 0.0599 \\
Station Random Effect & 1.9140 & 7.0000 & 1.6819 & 0.0176 \\
\hline
\end{tabular}

Table A3.92: Best productivity model for the Red-breasted Nuthatch (Sitta canadensis). The model was a quasi-Poisson GAMM with log link function. Based on AIC/QIC, support for including the noise level as a predictor of productivity was: none; support for including the standard deviation of noise was: weak. 


\begin{tabular}{lrrrr}
\hline A. parametric coefficients & Estimate & Std. Error & t-value & p-value \\
Intercept & -3.0317 & 1.4887 & -2.0365 & 0.0418 \\
Noise Level & -0.1285 & 0.0439 & -2.9274 & 0.0035 \\
Noise Standard Deviation & 0.5041 & 0.1954 & 2.5798 & 0.0100 \\
PC 1 & -0.0547 & 0.1515 & -0.3612 & 0.7180 \\
PC 2 & -0.1281 & 0.1124 & -1.1396 & 0.2546 \\
PC 3 & -0.1776 & 0.1075 & -1.6525 & 0.0986 \\
PC 4 & -0.0421 & 0.1660 & -0.2538 & 0.7997 \\
PC 5 & 0.5623 & 0.1892 & 2.9716 & 0.0030 \\
PC 6 & 0.0131 & 0.1443 & 0.0908 & 0.9277 \\
PC 7 & -0.0594 & 0.1414 & -0.4204 & 0.6742 \\
PC 8 & -0.0022 & 0.2943 & -0.0076 & 0.9939 \\
\hline B. smooth terms & edf & Ref.df & F-value & p-value \\
Location Smooth & 1.4012 & 19.0000 & 0.6237 & 0.3604 \\
Year Smooth & 6.2264 & 7.0000 & 15.0518 & $<0.0001$ \\
Station Random Effect & 131.5414 & 195.0000 & 3.2410 & $<0.0001$ \\
\hline
\end{tabular}

Table A3.93: Best abundance model for the Red-breasted Nuthatch (Sitta canadensis). The model was a quasi-Poisson GAMM with log link function. Based on AIC/QIC, support for including the noise level as a predictor of abundance was: strong; support for including the standard deviation of noise was: strong.

\begin{tabular}{lrrrr}
\hline A. parametric coefficients & Estimate & Std. Error & t-value & p-value \\
Intercept & -3.3188 & 1.0963 & -3.0273 & 0.0027 \\
PC 1 & -0.2990 & 0.8248 & -0.3625 & 0.7172 \\
PC 2 & -0.8040 & 0.8737 & -0.9203 & 0.3582 \\
PC 3 & 0.3738 & 0.5742 & 0.6509 & 0.5156 \\
PC 4 & -1.3038 & 1.6947 & -0.7693 & 0.4423 \\
PC 5 & 2.7917 & 2.1608 & 1.2920 & 0.1974 \\
PC 6 & -0.9824 & 1.1654 & -0.8429 & 0.3999 \\
PC 7 & 0.8947 & 1.4728 & 0.6075 & 0.5440 \\
PC 8 & -0.9044 & 1.8349 & -0.4929 & 0.6224 \\
\hline B. smooth terms & edf & Ref.df & F-value & p-value \\
Location Smooth & 25.8915 & 81.0000 & 1.9307 & $<0.0001$ \\
Year Smooth & 1.5697 & 19.0000 & 2.3045 & 0.0591 \\
Station Random Effect & 4.7945 & 7.0000 & 8.0258 & 0.0001 \\
\hline
\end{tabular}

Table A3.94: Best productivity model for the Ruby-crowned Kinglet (Regulus calendula). The model was a quasi-Poisson GAMM with log link function. Based on AIC/QIC, support for including the noise level as a predictor of productivity was: none; support for including the standard deviation of noise was: none. 


\begin{tabular}{lrrrr}
\hline A. parametric coefficients & Estimate & Std. Error & t-value & p-value \\
Intercept & -7.3439 & 3.6606 & -2.0062 & 0.0451 \\
Noise Level & 0.0115 & 0.1076 & 0.1065 & 0.9152 \\
PC 1 & -0.6824 & 0.4277 & -1.5954 & 0.1110 \\
PC 2 & 0.5094 & 0.2767 & 1.8409 & 0.0660 \\
PC 3 & -0.0425 & 0.2123 & -0.2004 & 0.8412 \\
PC 4 & -0.2535 & 0.2626 & -0.9654 & 0.3346 \\
PC 5 & 0.2088 & 0.3314 & 0.6301 & 0.5288 \\
PC 6 & -0.2690 & 0.3432 & -0.7839 & 0.4333 \\
PC 7 & -0.2304 & 0.3384 & -0.6808 & 0.4962 \\
PC 8 & -0.5516 & 1.1046 & -0.4993 & 0.6177 \\
\hline B. smooth terms & edf & Ref.df & F-value & p-value \\
Location Smooth & 0.8484 & 19.0000 & 3.9138 & 0.1781 \\
Year Smooth & 5.6430 & 7.0000 & 28.5318 & $<0.0001$ \\
Station Random Effect & 65.1373 & 81.0000 & 9.8695 & $<0.0001$ \\
\hline
\end{tabular}

Table A3.95: Best abundance model for the Ruby-crowned Kinglet (Regulus calendula). The model was a quasi-Poisson GAMM with log link function. Based on AIC/QIC, support for including the noise level as a predictor of abundance was: weak; support for including the standard deviation of noise was: none.

\begin{tabular}{lrrrr}
\hline A. parametric coefficients & Estimate & Std. Error & t-value & p-value \\
Intercept & -4.3790 & 1.8105 & -2.4187 & 0.0157 \\
Noise Level & 0.0168 & 0.0443 & 0.3784 & 0.7052 \\
Noise Standard Deviation & 0.3250 & 0.2759 & 1.1783 & 0.2388 \\
PC 1 & -0.3899 & 0.2257 & -1.7277 & 0.0842 \\
PC 2 & 0.0728 & 0.1713 & 0.4248 & 0.6710 \\
PC 3 & -0.3914 & 0.3356 & -1.1663 & 0.2436 \\
PC 4 & 0.0722 & 0.1685 & 0.4286 & 0.6683 \\
PC 5 & 0.0456 & 0.1933 & 0.2357 & 0.8137 \\
PC 6 & 0.3586 & 0.2929 & 1.2245 & 0.2209 \\
PC 7 & -0.0470 & 0.1811 & -0.2598 & 0.7950 \\
PC 8 & -0.4567 & 0.3605 & -1.2670 & 0.2053 \\
\hline B. smooth terms & edf & Ref.df & F-value & p-value \\
Location Smooth & 159.8349 & 437.0000 & 0.6054 & $<0.0001$ \\
Year Smooth & 9.7350 & 19.0000 & 14.4904 & 0.1531 \\
Station Random Effect & 5.1152 & 7.0000 & 7.3138 & 0.0151 \\
\hline
\end{tabular}

Table A3.96: Best productivity model for the Red-eyed Vireo (Vireo olivaceus). The model was a quasi-Poisson GAMM with log link function. Based on AIC/QIC, support for including the noise level as a predictor of productivity was: weak; support for including the standard deviation of noise was: strong. 


\begin{tabular}{lrrrr}
\hline A. parametric coefficients & Estimate & Std. Error & t-value & p-value \\
Intercept & -5.7876 & 0.9902 & -5.8449 & $<0.0001$ \\
Noise Level & -0.0055 & 0.0252 & -0.2196 & 0.8262 \\
Noise Standard Deviation & 0.3111 & 0.1434 & 2.1695 & 0.0301 \\
PC 1 & -0.1688 & 0.0786 & -2.1475 & 0.0318 \\
PC 2 & -0.1100 & 0.0670 & -1.6413 & 0.1008 \\
PC 3 & -0.2414 & 0.1285 & -1.8782 & 0.0604 \\
PC 4 & 0.1448 & 0.0854 & 1.6951 & 0.0901 \\
PC 5 & 0.2553 & 0.0978 & 2.6097 & 0.0091 \\
PC 6 & -0.0248 & 0.1169 & -0.2118 & 0.8323 \\
PC 7 & -0.0186 & 0.0848 & -0.2192 & 0.8265 \\
PC 8 & 0.0013 & 0.0939 & 0.0141 & 0.9887 \\
\hline B. smooth terms & edf & Ref.df & F-value & p-value \\
Location Smooth & 11.8426 & 19.0000 & 436.1807 & 0.0083 \\
Year Smooth & 4.4853 & 7.0000 & 49.9088 & $<0.0001$ \\
Station Random Effect & 337.7243 & 440.0000 & 6.5305 & $<0.0001$ \\
\hline
\end{tabular}

Table A3.97: Best abundance model for the Red-eyed Vireo (Vireo olivaceus). The model was a quasi-Poisson GAMM with log link function. Based on AIC/QIC, support for including the noise level as a predictor of abundance was: weak; support for including the standard deviation of noise was: strong. 


\begin{tabular}{lrrrr}
\hline A. parametric coefficients & Estimate & Std. Error & t-value & p-value \\
Intercept & -0.1031 & 1.8774 & -0.0549 & 0.9562 \\
Noise Level & -0.0153 & 0.0515 & -0.2972 & 0.7664 \\
Noise Standard Deviation & -0.1737 & 0.2510 & -0.6920 & 0.4891 \\
PC 1 & -0.2490 & 0.2301 & -1.0819 & 0.2796 \\
PC 2 & 0.2931 & 0.1462 & 2.0042 & 0.0454 \\
PC 3 & -0.2156 & 0.1743 & -1.2365 & 0.2166 \\
PC 4 & 0.1487 & 0.1493 & 0.9966 & 0.3193 \\
PC 5 & -0.3093 & 0.1748 & -1.7690 & 0.0773 \\
PC 6 & -0.7808 & 0.2958 & -2.6394 & 0.0085 \\
PC 7 & 0.0083 & 0.1689 & 0.0493 & 0.9607 \\
PC 8 & -0.5969 & 0.4316 & -1.3830 & 0.1670 \\
\hline B. smooth terms & edf & Ref.df & F-value & p-value \\
Location Smooth & 46.9424 & 124.0000 & 1.8257 & $<0.0001$ \\
Year Smooth & 8.1167 & 19.0000 & 36.1522 & 0.0017 \\
Station Random Effect & 0.0440 & 7.0000 & 0.0061 & 0.3389 \\
\hline
\end{tabular}

Table A3.98: Best productivity model for the Rufous Hummingbird (Selasphorus rufus). The model was a quasi-Poisson GAMM with log link function. Based on AIC/QIC, support for including the noise level as a predictor of productivity was: weak; support for including the standard deviation of noise was: weak. 


\begin{tabular}{lrrrr}
\hline A. parametric coefficients & Estimate & Std. Error & t-value & p-value \\
Intercept & -5.6264 & 0.1366 & -41.2015 & $<0.0001$ \\
PC 1 & 0.0348 & 0.1743 & 0.1998 & 0.8417 \\
PC 2 & -0.2080 & 0.1410 & -1.4756 & 0.1403 \\
PC 3 & 0.1117 & 0.1343 & 0.8314 & 0.4059 \\
PC 4 & 0.1091 & 0.1345 & 0.8111 & 0.4174 \\
PC 5 & -0.0646 & 0.1469 & -0.4399 & 0.6601 \\
PC 6 & -0.3158 & 0.1834 & -1.7222 & 0.0852 \\
PC 7 & 0.0601 & 0.1722 & 0.3492 & 0.7270 \\
PC 8 & 0.2897 & 0.4640 & 0.6243 & 0.5325 \\
\hline B. smooth terms & edf & Ref.df & F-value & p-value \\
Location Smooth & 8.5769 & 19.0000 & 165.4871 & 0.0150 \\
Year Smooth & 5.2671 & 7.0000 & 18.4449 & $<0.0001$ \\
Station Random Effect & 92.3668 & 139.0000 & 3.8574 & $<0.0001$ \\
\hline
\end{tabular}

Table A3.99: Best abundance model for the Rufous Hummingbird (Selasphorus rufus). The model was a quasi-Poisson GAMM with log link function. Based on AIC/QIC, support for including the noise level as a predictor of abundance was: none; support for including the standard deviation of noise was: none.

\begin{tabular}{lrrrr}
\hline A. parametric coefficients & Estimate & Std. Error & t-value & p-value \\
Intercept & -3.7821 & 2.0679 & -1.8290 & 0.0678 \\
PC 1 & -0.1069 & 0.6877 & -0.1555 & 0.8765 \\
PC 2 & -0.0375 & 0.8162 & -0.0460 & 0.9634 \\
PC 3 & -0.4498 & 2.4828 & -0.1812 & 0.8563 \\
PC 4 & -0.8354 & 0.8462 & -0.9871 & 0.3239 \\
PC 5 & 0.4943 & 0.9692 & 0.5101 & 0.6102 \\
PC 6 & 1.4105 & 2.1112 & 0.6681 & 0.5043 \\
PC 7 & -0.5916 & 1.0214 & -0.5793 & 0.5626 \\
PC 8 & -0.0256 & 1.1842 & -0.0216 & 0.9827 \\
\hline B. smooth terms & edf & Ref.df & F-value & p-value \\
Location Smooth & 92.6425 & 199.0000 & 0.6598 & 0.0007 \\
Year Smooth & 4.5153 & 19.0000 & 18.6423 & 0.3141 \\
Station Random Effect & 1.3363 & 7.0000 & 1.8350 & 0.2540 \\
\hline
\end{tabular}

Table A3.100: Best productivity model for the Scarlet Tanager (Piranga olivacea). The model was a quasi-Poisson GAMM with log link function. Based on AIC/QIC, support for including the noise level as a predictor of productivity was: none; support for including the standard deviation of noise was: none. 


\begin{tabular}{lrrrr}
\hline A. parametric coefficients & Estimate & Std. Error & t-value & p-value \\
Intercept & -4.9601 & 1.4352 & -3.4560 & 0.0006 \\
Noise Level & -0.0391 & 0.0339 & -1.1538 & 0.2487 \\
Noise Standard Deviation & -0.3710 & 0.1964 & -1.8887 & 0.0591 \\
PC 1 & -0.1434 & 0.1431 & -1.0021 & 0.3164 \\
PC 2 & -0.1472 & 0.1523 & -0.9662 & 0.3341 \\
PC 3 & -0.0609 & 0.4634 & -0.1315 & 0.8954 \\
PC 4 & 0.0471 & 0.1748 & 0.2697 & 0.7874 \\
PC 5 & 0.1269 & 0.2088 & 0.6077 & 0.5434 \\
PC 6 & 0.1340 & 0.4449 & 0.3011 & 0.7634 \\
PC 7 & -0.0498 & 0.1967 & -0.2534 & 0.8000 \\
PC 8 & -0.0392 & 0.1381 & -0.2837 & 0.7767 \\
\hline B. smooth terms & edf & Ref.df & F-value & p-value \\
Location Smooth & 9.9117 & 19.0000 & 16.7628 & 0.0626 \\
Year Smooth & 0.8590 & 7.0000 & 1.9617 & 0.0165 \\
Station Random Effect & 125.4142 & 210.0000 & 2.3140 & $<0.0001$ \\
\hline
\end{tabular}

Table A3.101: Best abundance model for the Scarlet Tanager (Piranga olivacea). The model was a quasi-Poisson GAMM with log link function. Based on AIC/QIC, support for including the noise level as a predictor of abundance was: strong; support for including the standard deviation of noise was: strong.

\begin{tabular}{lrrrr}
\hline A. parametric coefficients & Estimate & Std. Error & t-value & p-value \\
Intercept & -0.4620 & 0.0338 & -13.6707 & $<0.0001$ \\
PC 1 & 0.0185 & 0.0250 & 0.7382 & 0.4604 \\
PC 2 & 0.0190 & 0.0279 & 0.6816 & 0.4956 \\
PC 3 & -0.0568 & 0.0355 & -1.6015 & 0.1094 \\
PC 4 & -0.0318 & 0.0284 & -1.1220 & 0.2619 \\
PC 5 & 0.0687 & 0.0347 & 1.9786 & 0.0479 \\
PC 6 & -0.0044 & 0.0404 & -0.1092 & 0.9131 \\
PC 7 & -0.0299 & 0.0318 & -0.9405 & 0.3471 \\
PC 8 & -0.0212 & 0.0475 & -0.4459 & 0.6557 \\
\hline B. smooth terms & edf & Ref.df & F-value & p-value \\
Location Smooth & 221.6294 & 501.0000 & 2.8792 & $<0.0001$ \\
Year Smooth & 12.2336 & 19.0000 & 99.0189 & 0.0001 \\
Station Random Effect & 0.8993 & 7.0000 & 3.5169 & 0.0286 \\
\hline
\end{tabular}

Table A3.102: Best productivity model for the Song Sparrow (Melospiza melodia). The model was a quasi-Poisson GAMM with log link function. Based on AIC/QIC, support for including the noise level as a predictor of productivity was: none; support for including the standard deviation of noise was: none. 


\begin{tabular}{lrrrr}
\hline A. parametric coefficients & Estimate & Std. Error & t-value & p-value \\
Intercept & -4.9705 & 0.1582 & -31.4288 & $<0.0001$ \\
Noise Standard Deviation & 0.3443 & 0.1517 & 2.2696 & 0.0233 \\
PC 1 & 0.2476 & 0.0573 & 4.3251 & $<0.0001$ \\
PC 2 & 0.2925 & 0.0684 & 4.2775 & $<0.0001$ \\
PC 3 & -0.0538 & 0.0822 & -0.6544 & 0.5129 \\
PC 4 & -0.0543 & 0.0614 & -0.8838 & 0.3769 \\
PC 5 & 0.0246 & 0.0755 & 0.3258 & 0.7446 \\
PC 6 & -0.1522 & 0.0845 & -1.8015 & 0.0717 \\
PC 7 & 0.0054 & 0.0749 & 0.0715 & 0.9430 \\
PC 8 & -0.1360 & 0.1040 & -1.3079 & 0.1910 \\
\hline B. smooth terms & edf & Ref.df & F-value & p-value \\
Location Smooth & 13.4235 & 19.0000 & 17438.8744 & 0.0008 \\
Year Smooth & 6.5783 & 7.0000 & 1325.2390 & $<0.0001$ \\
Station Random Effect & 462.0270 & 537.0000 & 15.0428 & $<0.0001$ \\
\hline
\end{tabular}

Table A3.103: Best abundance model for the Song Sparrow (Melospiza melodia). The model was a quasi-Poisson GAMM with log link function. Based on AIC/QIC, support for including the noise level as a predictor of abundance was: none; support for including the standard deviation of noise was: weak.

\begin{tabular}{lrrrr}
\hline A. parametric coefficients & Estimate & Std. Error & t-value & p-value \\
Intercept & -0.8795 & 0.0766 & -11.4791 & $<0.0001$ \\
PC 1 & 0.0357 & 0.0553 & 0.6453 & 0.5188 \\
PC 2 & -0.0081 & 0.0634 & -0.1277 & 0.8984 \\
PC 3 & -0.0023 & 0.0554 & -0.0418 & 0.9667 \\
PC 4 & 0.1800 & 0.0567 & 3.1717 & 0.0016 \\
PC 5 & 0.1724 & 0.0636 & 2.7130 & 0.0068 \\
PC 6 & -0.0894 & 0.0604 & -1.4811 & 0.1388 \\
PC 7 & -0.0569 & 0.0752 & -0.7570 & 0.4492 \\
PC 8 & -0.0344 & 0.1421 & -0.2422 & 0.8087 \\
\hline B. smooth terms & edf & Ref.df & F-value & p-value \\
Location Smooth & 96.5385 & 231.0000 & 1.5137 & $<0.0001$ \\
Year Smooth & 8.3228 & 19.0000 & 24.2075 & 0.0009 \\
Station Random Effect & 3.0302 & 7.0000 & 3.5802 & 0.0097 \\
\hline
\end{tabular}

Table A3.104: Best productivity model for the Spotted Towhee (Pipilo maculatus). The model was a quasi-Poisson GAMM with log link function. Based on AIC/QIC, support for including the noise level as a predictor of productivity was: none; support for including the standard deviation of noise was: none. 


\begin{tabular}{lrrrr}
\hline A. parametric coefficients & Estimate & Std. Error & t-value & p-value \\
Intercept & -5.5179 & 0.1296 & -42.5655 & $<0.0001$ \\
PC 1 & 0.1514 & 0.0998 & 1.5180 & 0.1292 \\
PC 2 & 0.2132 & 0.1098 & 1.9417 & 0.0523 \\
PC 3 & 0.1607 & 0.0968 & 1.6613 & 0.0968 \\
PC 4 & -0.2725 & 0.0997 & -2.7326 & 0.0063 \\
PC 5 & -0.2340 & 0.1091 & -2.1442 & 0.0321 \\
PC 6 & 0.0553 & 0.1037 & 0.5330 & 0.5941 \\
PC 7 & -0.0112 & 0.1424 & -0.0789 & 0.9371 \\
PC 8 & -0.1483 & 0.2645 & -0.5607 & 0.5751 \\
\hline B. smooth terms & edf & Ref.df & F-value & p-value \\
Location Smooth & 8.3816 & 19.0000 & 3577.8848 & 0.0033 \\
Year Smooth & 5.3978 & 7.0000 & 59.6013 & 0.0228 \\
Station Random Effect & 219.8740 & 253.0000 & 9.8830 & $<0.0001$ \\
\hline
\end{tabular}

Table A3.105: Best abundance model for the Spotted Towhee (Pipilo maculatus). The model was a quasi-Poisson GAMM with log link function. Based on AIC/QIC, support for including the noise level as a predictor of abundance was: none; support for including the standard deviation of noise was: none.

\begin{tabular}{lrrrr}
\hline A. parametric coefficients & Estimate & Std. Error & t-value & p-value \\
Intercept & -3.5261 & 1.2473 & -2.8268 & 0.0048 \\
Noise Level & 0.0262 & 0.0343 & 0.7634 & 0.4453 \\
PC 1 & -0.2562 & 0.1345 & -1.9050 & 0.0569 \\
PC 2 & 0.3297 & 0.0869 & 3.7932 & 0.0002 \\
PC 3 & -0.1140 & 0.1051 & -1.0844 & 0.2783 \\
PC 4 & -0.0290 & 0.0916 & -0.3161 & 0.7520 \\
PC 5 & 0.1789 & 0.1048 & 1.7062 & 0.0881 \\
PC 6 & 0.1753 & 0.1054 & 1.6637 & 0.0963 \\
PC 7 & -0.0109 & 0.1031 & -0.1056 & 0.9159 \\
PC 8 & -0.7317 & 0.2231 & -3.2798 & 0.0011 \\
\hline B. smooth terms & edf & Ref.df & F-value & p-value \\
Location Smooth & 114.4531 & 297.0000 & 2.5796 & $<0.0001$ \\
Year Smooth & 8.1588 & 19.0000 & 38.5036 & 0.1043 \\
Station Random Effect & 6.8096 & 7.0000 & 18.9283 & 0.0035 \\
\hline
\end{tabular}

Table A3.106: Best productivity model for the Swainson's Thrush (Catharus ustulatus). The model was a quasi-Poisson GAMM with log link function. Based on AIC/QIC, support for including the noise level as a predictor of productivity was: strong; support for including the standard deviation of noise was: none. 


\begin{tabular}{lrrrr}
\hline A. parametric coefficients & Estimate & Std. Error & t-value & p-value \\
Intercept & -4.5971 & 0.0935 & -49.1437 & $<0.0001$ \\
PC 1 & -0.0505 & 0.0899 & -0.5623 & 0.5740 \\
PC 2 & -0.1435 & 0.0875 & -1.6408 & 0.1010 \\
PC 3 & -0.1396 & 0.0995 & -1.4036 & 0.1606 \\
PC 4 & 0.1015 & 0.0851 & 1.1927 & 0.2331 \\
PC 5 & 0.1633 & 0.0962 & 1.6971 & 0.0898 \\
PC 6 & 0.0006 & 0.1037 & 0.0063 & 0.9950 \\
PC 7 & 0.0189 & 0.1018 & 0.1852 & 0.8531 \\
PC 8 & 0.1454 & 0.2299 & 0.6322 & 0.5273 \\
\hline B. smooth terms & edf & Ref.df & F-value & p-value \\
Location Smooth & 16.3055 & 19.0000 & 49696.0184 & $<0.0001$ \\
Year Smooth & 3.3826 & 7.0000 & 105.3056 & $<0.0001$ \\
Station Random Effect & 226.0497 & 292.0000 & 15.4334 & $<0.0001$ \\
\hline
\end{tabular}

Table A3.107: Best abundance model for the Swainson's Thrush (Catharus ustulatus). The model was a quasi-Poisson GAMM with log link function. Based on AIC/QIC, support for including the noise level as a predictor of abundance was: none; support for including the standard deviation of noise was: none.

\begin{tabular}{lrrrr}
\hline A. parametric coefficients & Estimate & Std. Error & t-value & p-value \\
Intercept & -19.4998 & 26.7243 & -0.7297 & 0.4672 \\
Noise Level & -0.0587 & 0.2876 & -0.2040 & 0.8388 \\
Noise Standard Deviation & 0.2681 & 1.0622 & 0.2524 & 0.8012 \\
PC 1 & -5.9392 & 7.2985 & -0.8138 & 0.4176 \\
PC 2 & -6.2567 & 9.0408 & -0.6921 & 0.4904 \\
PC 3 & 6.9049 & 10.5445 & 0.6548 & 0.5140 \\
PC 4 & 18.1785 & 26.8155 & 0.6779 & 0.4993 \\
PC 5 & 19.4381 & 23.9317 & 0.8122 & 0.4184 \\
PC 6 & -0.8402 & 2.5095 & -0.3348 & 0.7384 \\
PC 7 & -2.5565 & 6.6066 & -0.3870 & 0.6995 \\
PC 8 & -1.4840 & 6.1840 & -0.2400 & 0.8108 \\
\hline B. smooth terms & edf & Ref.df & F-value & p-value \\
Location Smooth & 12.5627 & 46.0000 & 0.3080 & 0.2506 \\
Year Smooth & 1.2056 & 19.0000 & 0.1120 & 0.3819 \\
Station Random Effect & 0.1249 & 7.0000 & 0.0088 & 0.4987 \\
\hline
\end{tabular}

Table A3.108: Best productivity model for the Townsend's Solitaire (Myadestes townsendi). The model was a quasi-Poisson GAMM with log link function. Based on AIC/QIC, support for including the noise level as a predictor of productivity was: weak; support for including the standard deviation of noise was: strong. 


\begin{tabular}{lrrrr}
\hline A. parametric coefficients & Estimate & Std. Error & t-value & p-value \\
Intercept & -9.2530 & 1.6160 & -5.7258 & $<0.0001$ \\
Noise Standard Deviation & 0.6039 & 0.4194 & 1.4398 & 0.1503 \\
PC 1 & 0.8327 & 0.6279 & 1.3263 & 0.1851 \\
PC 2 & -1.1717 & 0.6697 & -1.7497 & 0.0805 \\
PC 3 & 0.7224 & 0.6592 & 1.0960 & 0.2734 \\
PC 4 & 0.9401 & 1.7056 & 0.5512 & 0.5816 \\
PC 5 & 1.1912 & 1.4819 & 0.8039 & 0.4217 \\
PC 6 & -0.4497 & 0.4918 & -0.9143 & 0.3608 \\
PC 7 & 0.3005 & 0.9146 & 0.3286 & 0.7425 \\
PC 8 & 3.2303 & 1.5124 & 2.1358 & 0.0330 \\
\hline B. smooth terms & edf & Ref.df & F-value & p-value \\
Location Smooth & 5.4441 & 19.0000 & 2.1846 & 0.3619 \\
Year Smooth & 0.4945 & 7.0000 & 0.0732 & 0.3396 \\
Station Random Effect & 29.5461 & 61.0000 & 0.8155 & 0.0007 \\
\hline
\end{tabular}

Table A3.109: Best abundance model for the Townsend's Solitaire (Myadestes townsendi). The model was a quasi-Poisson GAMM with log link function. Based on AIC/QIC, support for including the noise level as a predictor of abundance was: none; support for including the standard deviation of noise was: strong.

\begin{tabular}{lrrrr}
\hline A. parametric coefficients & Estimate & Std. Error & t-value & p-value \\
Intercept & 6.4376 & 13.3176 & 0.4834 & 0.6292 \\
Noise Level & -0.3582 & 0.3263 & -1.0980 & 0.2730 \\
Noise Standard Deviation & 1.1764 & 0.9687 & 1.2144 & 0.2255 \\
PC 1 & -5.7519 & 10.9903 & -0.5234 & 0.6011 \\
PC 2 & 3.5773 & 6.2433 & 0.5730 & 0.5671 \\
PC 3 & -1.6402 & 1.6747 & -0.9794 & 0.3281 \\
PC 4 & -1.6770 & 2.5976 & -0.6456 & 0.5190 \\
PC 5 & 0.1870 & 1.2545 & 0.1491 & 0.8816 \\
PC 6 & 0.4076 & 2.5961 & 0.1570 & 0.8753 \\
PC 7 & 1.8077 & 1.4105 & 1.2816 & 0.2009 \\
PC 8 & -3.4752 & 16.4894 & -0.2108 & 0.8332 \\
\hline B. smooth terms & edf & Ref.df & F-value & p-value \\
Location Smooth & 5.1857 & 31.0000 & 0.6496 & $<0.0001$ \\
Year Smooth & 4.3481 & 19.0000 & 2.2009 & 0.0410 \\
Station Random Effect & 2.1582 & 7.0000 & 5.7985 & $<0.0001$ \\
\hline
\end{tabular}

Table A3.110: Best productivity model for the Townsend's Warbler (Setophaga townsendi). The model was a quasi-Poisson GAMM with log link function. Based on AIC/QIC, support for including the noise level as a predictor of productivity was: weak; support for including the standard deviation of noise was: weak. 


\begin{tabular}{lrrrr}
\hline A. parametric coefficients & Estimate & Std. Error & t-value & p-value \\
Intercept & -8.9989 & 1.2818 & -7.0206 & $<0.0001$ \\
Noise Standard Deviation & 0.1366 & 0.4650 & 0.2938 & 0.7690 \\
PC 1 & -3.0628 & 2.2303 & -1.3733 & 0.1702 \\
PC 2 & 0.2351 & 1.3783 & 0.1706 & 0.8646 \\
PC 3 & -0.0318 & 0.3711 & -0.0857 & 0.9317 \\
PC 4 & 0.2339 & 0.6570 & 0.3560 & 0.7220 \\
PC 5 & -0.0843 & 0.8217 & -0.1026 & 0.9183 \\
PC 6 & 1.1610 & 0.7033 & 1.6508 & 0.0993 \\
PC 7 & 0.3909 & 0.4082 & 0.9574 & 0.3387 \\
PC 8 & -3.4977 & 3.6735 & -0.9521 & 0.3414 \\
\hline B. smooth terms & edf & Ref.df & F-value & p-value \\
Location Smooth & 11.3837 & 19.0000 & 88.9604 & 0.0010 \\
Year Smooth & 4.8972 & 7.0000 & 10.2192 & $<0.0001$ \\
Station Random Effect & 13.3808 & 38.0000 & 2.6032 & $<0.0001$ \\
\hline
\end{tabular}

Table A3.111: Best abundance model for the Townsend's Warbler (Setophaga townsendi). The model was a quasi-Poisson GAMM with log link function. Based on AIC/QIC, support for including the noise level as a predictor of abundance was: none; support for including the standard deviation of noise was: weak.

\begin{tabular}{lrrrr}
\hline A. parametric coefficients & Estimate & Std. Error & t-value & p-value \\
Intercept & -2.7312 & 0.1124 & -24.3070 & $<0.0001$ \\
PC 1 & -0.0075 & 0.1067 & -0.0700 & 0.9442 \\
PC 2 & 0.0235 & 0.0996 & 0.2360 & 0.8134 \\
PC 3 & -0.1259 & 0.0872 & -1.4442 & 0.1488 \\
PC 4 & -0.0849 & 0.1131 & -0.7512 & 0.4526 \\
PC 5 & 0.2074 & 0.1298 & 1.5977 & 0.1103 \\
PC 6 & 0.0772 & 0.1118 & 0.6900 & 0.4903 \\
PC 7 & 0.1984 & 0.1190 & 1.6670 & 0.0957 \\
PC 8 & -0.2896 & 0.2735 & -1.0590 & 0.2897 \\
\hline B. smooth terms & edf & Ref.df & F-value & p-value \\
Location Smooth & 99.7835 & 360.0000 & 0.8811 & $<0.0001$ \\
Year Smooth & 10.1708 & 19.0000 & 25.7076 & 0.0257 \\
Station Random Effect & 1.6555 & 7.0000 & 1.1432 & 0.0451 \\
\hline
\end{tabular}

Table A3.112: Best productivity model for the Warbling Vireo (Vireo gilvus). The model was a quasi-Poisson GAMM with log link function. Based on AIC/QIC, support for including the noise level as a predictor of productivity was: none; support for including the standard deviation of noise was: none. 


\begin{tabular}{lrrrr}
\hline A. parametric coefficients & Estimate & Std. Error & t-value & p-value \\
Intercept & -2.9069 & 1.1482 & -2.5317 & 0.0114 \\
Noise Level & -0.0828 & 0.0332 & -2.4919 & 0.0128 \\
Noise Standard Deviation & 0.2944 & 0.1471 & 2.0015 & 0.0454 \\
PC 1 & -0.0590 & 0.1121 & -0.5260 & 0.5989 \\
PC 2 & -0.2455 & 0.0753 & -3.2619 & 0.0011 \\
PC 3 & 0.0046 & 0.0665 & 0.0685 & 0.9454 \\
PC 4 & 0.0318 & 0.0693 & 0.4585 & 0.6466 \\
PC 5 & -0.0731 & 0.0785 & -0.9317 & 0.3516 \\
PC 6 & -0.0596 & 0.0795 & -0.7498 & 0.4534 \\
PC 7 & 0.0364 & 0.0881 & 0.4133 & 0.6794 \\
PC 8 & 0.1677 & 0.2301 & 0.7289 & 0.4661 \\
\hline B. smooth terms & edf & Ref.df & F-value & p-value \\
Location Smooth & 7.9826 & 19.0000 & 382.9377 & 0.1564 \\
Year Smooth & 3.4419 & 7.0000 & 11.2999 & 0.0249 \\
Station Random Effect & 281.9414 & 361.0000 & 9.2574 & $<0.0001$ \\
\hline
\end{tabular}

Table A3.113: Best abundance model for the Warbling Vireo (Vireo gilvus). The model was a quasi-Poisson GAMM with log link function. Based on AIC/QIC, support for including the noise level as a predictor of abundance was: weak; support for including the standard deviation of noise was: weak.

\begin{tabular}{lrrrr}
\hline A. parametric coefficients & Estimate & Std. Error & t-value & p-value \\
Intercept & -0.9886 & 1.5129 & -0.6534 & 0.5137 \\
Noise Level & -0.0162 & 0.0398 & -0.4069 & 0.6842 \\
PC 1 & 0.1949 & 0.1294 & 1.5060 & 0.1325 \\
PC 2 & 0.1849 & 0.1051 & 1.7589 & 0.0790 \\
PC 3 & 0.1179 & 0.1045 & 1.1279 & 0.2597 \\
PC 4 & 0.1170 & 0.1508 & 0.7758 & 0.4381 \\
PC 5 & -0.2917 & 0.1825 & -1.5980 & 0.1105 \\
PC 6 & 0.0954 & 0.1361 & 0.7012 & 0.4834 \\
PC 7 & -0.1562 & 0.1447 & -1.0794 & 0.2808 \\
PC 8 & 0.1191 & 0.1612 & 0.7391 & 0.4601 \\
\hline B. smooth terms & edf & Ref.df & F-value & p-value \\
Location Smooth & 93.4962 & 291.0000 & 0.5148 & $<0.0001$ \\
Year Smooth & 0.0004 & 19.0000 & 0.0000 & 0.5721 \\
Station Random Effect & 1.5168 & 7.0000 & 0.9737 & 0.2442 \\
\hline
\end{tabular}

Table A3.114: Best productivity model for the White-breasted Nuthatch (Sitta carolinensis). The model was a quasi-Poisson GAMM with log link function. Based on AIC/QIC, support for including the noise level as a predictor of productivity was: strong; support for including the standard deviation of noise was: none. 


\begin{tabular}{lrrrr}
\hline A. parametric coefficients & Estimate & Std. Error & t-value & p-value \\
Intercept & -8.8815 & 1.3480 & -6.5888 & $<0.0001$ \\
Noise Level & 0.0420 & 0.0351 & 1.1944 & 0.2324 \\
PC 1 & 0.0025 & 0.1038 & 0.0238 & 0.9810 \\
PC 2 & 0.0461 & 0.0784 & 0.5871 & 0.5572 \\
PC 3 & 0.0764 & 0.0867 & 0.8808 & 0.3785 \\
PC 4 & -0.0181 & 0.1133 & -0.1599 & 0.8730 \\
PC 5 & -0.0012 & 0.1361 & -0.0085 & 0.9932 \\
PC 6 & 0.0509 & 0.0999 & 0.5100 & 0.6101 \\
PC 7 & 0.1477 & 0.1053 & 1.4035 & 0.1606 \\
PC 8 & -0.1605 & 0.1154 & -1.3906 & 0.1644 \\
\hline B. smooth terms & edf & Ref.df & F-value & p-value \\
Location Smooth & 9.5619 & 19.0000 & 76.9712 & $<0.0001$ \\
Year Smooth & 2.4944 & 7.0000 & 25.6628 & $<0.0001$ \\
Station Random Effect & 206.6127 & 339.0000 & 1.8534 & $<0.0001$ \\
\hline
\end{tabular}

Table A3.115: Best abundance model for the White-breasted Nuthatch (Sitta carolinensis). The model was a quasi-Poisson GAMM with log link function. Based on AIC/QIC, support for including the noise level as a predictor of abundance was: strong; support for including the standard deviation of noise was: none.

\begin{tabular}{lrrrr}
\hline A. parametric coefficients & Estimate & Std. Error & t-value & p-value \\
Intercept & -8.1209 & 1.2338 & -6.5819 & $<0.0001$ \\
Noise Standard Deviation & 0.3087 & 0.9149 & 0.3375 & 0.7360 \\
PC 1 & 1.3044 & 1.3451 & 0.9697 & 0.3329 \\
PC 2 & -1.0761 & 0.9374 & -1.1480 & 0.2519 \\
PC 3 & 0.8044 & 0.4835 & 1.6637 & 0.0972 \\
PC 4 & 1.5727 & 1.2159 & 1.2934 & 0.1969 \\
PC 5 & 0.8924 & 0.9703 & 0.9198 & 0.3584 \\
PC 6 & -0.2170 & 0.9355 & -0.2320 & 0.8167 \\
PC 7 & -0.0007 & 1.2976 & -0.0005 & 0.9996 \\
PC 8 & 4.9846 & 4.3721 & 1.1401 & 0.2551 \\
\hline B. smooth terms & edf & Ref.df & F-value & p-value \\
Location Smooth & 0.0000 & 19.0000 & 0.0000 & 0.9136 \\
Year Smooth & 0.0004 & 7.0000 & 0.0000 & 0.5430 \\
Station Random Effect & 11.6486 & 20.0000 & 2.4616 & $<0.0001$ \\
\hline
\end{tabular}

Table A3.116: Best abundance model for the White-crowned Sparrow (Zonotrichia leucophrys). The model was a quasi-Poisson GAMM with log link function. Based on AIC/QIC, support for including the noise level as a predictor of abundance was: none; support for including the standard deviation of noise was: weak. 


\begin{tabular}{lrrrr}
\hline A. parametric coefficients & Estimate & Std. Error & t-value & p-value \\
Intercept & -3.2890 & 2.3336 & -1.4094 & 0.1611 \\
Noise Level & 0.0694 & 0.0684 & 1.0146 & 0.3122 \\
PC 1 & 0.0686 & 0.3709 & 0.1850 & 0.8535 \\
PC 2 & 0.3671 & 0.2023 & 1.8150 & 0.0718 \\
PC 3 & 0.0395 & 0.2005 & 0.1970 & 0.8441 \\
PC 4 & -0.1296 & 0.2314 & -0.5601 & 0.5764 \\
PC 5 & 0.0416 & 0.1364 & 0.3052 & 0.7607 \\
PC 6 & -0.3727 & 0.1835 & -2.0313 & 0.0443 \\
PC 7 & -0.2685 & 0.3529 & -0.7608 & 0.4481 \\
PC 8 & 0.9051 & 0.7802 & 1.1601 & 0.2481 \\
\hline B. smooth terms & edf & Ref.df & F-value & p-value \\
Location Smooth & 0.0004 & 34.0000 & 0.0000 & 0.1209 \\
Year Smooth & 4.1351 & 19.0000 & 1.0693 & 0.0001 \\
Station Random Effect & 0.0000 & 7.0000 & 0.0000 & 1.0000 \\
\hline
\end{tabular}

Table A3.117: Best productivity model for the Western Bluebird (Sialia mexicana). The model was a quasi-Poisson GAMM with log link function. Based on AIC/QIC, support for including the noise level as a predictor of productivity was: weak; support for including the standard deviation of noise was: none. 


\begin{tabular}{lrrrr}
\hline A. parametric coefficients & Estimate & Std. Error & t-value & p-value \\
Intercept & -5.9412 & 0.5631 & -10.5502 & $<0.0001$ \\
Noise Standard Deviation & -1.1724 & 0.5748 & -2.0395 & 0.0421 \\
PC 1 & 0.9621 & 0.3748 & 2.5671 & 0.0106 \\
PC 2 & -0.1795 & 0.2459 & -0.7298 & 0.4659 \\
PC 3 & 0.3933 & 0.2161 & 1.8201 & 0.0695 \\
PC 4 & -0.0647 & 0.2459 & -0.2632 & 0.7925 \\
PC 5 & 0.0660 & 0.1929 & 0.3422 & 0.7324 \\
PC 6 & -0.5090 & 0.2328 & -2.1863 & 0.0294 \\
PC 7 & -0.5776 & 0.3740 & -1.5443 & 0.1233 \\
PC 8 & 1.3740 & 0.9253 & 1.4849 & 0.1384 \\
\hline B. smooth terms & edf & Ref.df & F-value & p-value \\
Location Smooth & 1.3985 & 19.0000 & 3.1992 & 0.0580 \\
Year Smooth & 2.0950 & 7.0000 & 4.8361 & 0.0334 \\
Station Random Effect & 25.1865 & 39.0000 & 2.7892 & $<0.0001$ \\
\hline
\end{tabular}

Table A3.118: Best abundance model for the Western Bluebird (Sialia mexicana). The model was a quasi-Poisson GAMM with log link function. Based on AIC/QIC, support for including the noise level as a predictor of abundance was: none; support for including the standard deviation of noise was: strong.

\begin{tabular}{lrrrr}
\hline A. parametric coefficients & Estimate & Std. Error & t-value & p-value \\
Intercept & -2.3187 & 0.3985 & -5.8179 & $<0.0001$ \\
Noise Standard Deviation & 0.2665 & 0.2107 & 1.2650 & 0.2068 \\
PC 1 & 0.0469 & 0.1188 & 0.3947 & 0.6933 \\
PC 2 & -0.2794 & 0.2489 & -1.1222 & 0.2626 \\
PC 3 & 0.3041 & 0.1884 & 1.6146 & 0.1074 \\
PC 4 & 0.2178 & 0.1199 & 1.8171 & 0.0701 \\
PC 5 & -0.4322 & 0.2215 & -1.9513 & 0.0519 \\
PC 6 & 0.1698 & 0.1938 & 0.8761 & 0.3816 \\
PC 7 & 0.4670 & 0.2402 & 1.9441 & 0.0527 \\
PC 8 & -0.2683 & 0.2051 & -1.3080 & 0.1918 \\
\hline B. smooth terms & edf & Ref.df & F-value & p-value \\
Location Smooth & 10.4454 & 119.0000 & 0.0905 & 0.3514 \\
Year Smooth & 3.0656 & 19.0000 & 0.8128 & 0.0062 \\
Station Random Effect & 2.0312 & 7.0000 & 1.1672 & 0.0231 \\
\hline
\end{tabular}

Table A3.119: Best productivity model for the Western Scrub-Jay (Aphelocoma californica). The model was a quasi-Poisson GAMM with log link function. Based on AIC/QIC, support for including the noise level as a predictor of productivity was: none; support for including the standard deviation of noise was: strong. 


\begin{tabular}{lrrrr}
\hline A. parametric coefficients & Estimate & Std. Error & t-value & p-value \\
Intercept & -8.5727 & 1.0421 & -8.2260 & $<0.0001$ \\
Noise Level & 0.0355 & 0.0281 & 1.2617 & 0.2074 \\
Noise Standard Deviation & 0.1155 & 0.1751 & 0.6598 & 0.5096 \\
PC 1 & 0.1653 & 0.1039 & 1.5915 & 0.1118 \\
PC 2 & 0.0066 & 0.1124 & 0.0588 & 0.9531 \\
PC 3 & 0.1697 & 0.0843 & 2.0135 & 0.0444 \\
PC 4 & -0.2264 & 0.0823 & -2.7490 & 0.0061 \\
PC 5 & -0.0130 & 0.0954 & -0.1363 & 0.8916 \\
PC 6 & 0.1111 & 0.0930 & 1.1947 & 0.2325 \\
PC 7 & -0.2176 & 0.1281 & -1.6989 & 0.0897 \\
PC 8 & -0.2603 & 0.1598 & -1.6286 & 0.1037 \\
\hline B. smooth terms & edf & Ref.df & F-value & p-value \\
Location Smooth & 0.0003 & 19.0000 & 0.0000 & 0.8736 \\
Year Smooth & 0.0010 & 7.0000 & 0.0001 & 0.5418 \\
Station Random Effect & 80.2528 & 129.0000 & 2.2458 & $<0.0001$ \\
\hline
\end{tabular}

Table A3.120: Best abundance model for the Western Scrub-Jay (Aphelocoma californica). The model was a quasi-Poisson GAMM with log link function. Based on AIC/QIC, support for including the noise level as a predictor of abundance was: strong; support for including the standard deviation of noise was: weak.

\begin{tabular}{lrrrr}
\hline A. parametric coefficients & Estimate & Std. Error & t-value & p-value \\
Intercept & -5.0253 & 2.0596 & -2.4399 & 0.0148 \\
Noise Level & 0.0849 & 0.0592 & 1.4344 & 0.1517 \\
PC 1 & -0.2706 & 0.2110 & -1.2827 & 0.1998 \\
PC 2 & 0.1041 & 0.1728 & 0.6025 & 0.5470 \\
PC 3 & -0.1394 & 0.1391 & -1.0019 & 0.3166 \\
PC 4 & -0.2267 & 0.2032 & -1.1161 & 0.2646 \\
PC 5 & 0.2768 & 0.2321 & 1.1928 & 0.2331 \\
PC 6 & -0.0400 & 0.1888 & -0.2117 & 0.8324 \\
PC 7 & 0.4216 & 0.3032 & 1.3905 & 0.1646 \\
PC 8 & -0.4597 & 0.3855 & -1.1926 & 0.2332 \\
\hline B. smooth terms & edf & Ref.df & F-value & p-value \\
Location Smooth & 106.1816 & 276.0000 & 1.2605 & $<0.0001$ \\
Year Smooth & 9.4893 & 19.0000 & 101.5618 & 0.0066 \\
Station Random Effect & 6.3415 & 7.0000 & 10.0046 & $<0.0001$ \\
\hline
\end{tabular}

Table A3.121: Best productivity model for the Western Tanager (Piranga ludoviciana). The model was a quasi-Poisson GAMM with log link function. Based on AIC/QIC, support for including the noise level as a predictor of productivity was: weak; support for including the standard deviation of noise was: none. 


\begin{tabular}{lrrrr}
\hline A. parametric coefficients & Estimate & Std. Error & t-value & p-value \\
Intercept & -6.1426 & 0.1870 & -32.8461 & $<0.0001$ \\
Noise Standard Deviation & 0.2729 & 0.1663 & 1.6407 & 0.1010 \\
PC 1 & -0.2117 & 0.1072 & -1.9742 & 0.0485 \\
PC 2 & -0.1026 & 0.1044 & -0.9820 & 0.3262 \\
PC 3 & 0.0241 & 0.0850 & 0.2836 & 0.7767 \\
PC 4 & -0.0480 & 0.1131 & -0.4247 & 0.6711 \\
PC 5 & 0.0142 & 0.1093 & 0.1303 & 0.8963 \\
PC 6 & -0.0168 & 0.1100 & -0.1529 & 0.8785 \\
PC 7 & -0.0809 & 0.1667 & -0.4853 & 0.6275 \\
PC 8 & 0.1342 & 0.2585 & 0.5189 & 0.6039 \\
\hline B. smooth terms & edf & Ref.df & F-value & p-value \\
Location Smooth & 10.5284 & 19.0000 & 201.1588 & 0.0226 \\
Year Smooth & 5.3334 & 7.0000 & 48.8426 & $<0.0001$ \\
Station Random Effect & 196.3052 & 275.0000 & 6.4981 & $<0.0001$ \\
\hline
\end{tabular}

Table A3.122: Best abundance model for the Western Tanager (Piranga ludoviciana). The model was a quasi-Poisson GAMM with log link function. Based on AIC/QIC, support for including the noise level as a predictor of abundance was: none; support for including the standard deviation of noise was: weak.

\begin{tabular}{lrrrr}
\hline A. parametric coefficients & Estimate & Std. Error & t-value & p-value \\
Intercept & 0.8922 & 1.1108 & 0.8032 & 0.4220 \\
Noise Level & -0.0626 & 0.0276 & -2.2705 & 0.0233 \\
Noise Standard Deviation & 0.2701 & 0.1673 & 1.6148 & 0.1066 \\
PC 1 & 0.1043 & 0.1419 & 0.7350 & 0.4625 \\
PC 2 & -0.0656 & 0.1029 & -0.6377 & 0.5238 \\
PC 3 & 0.2398 & 0.0977 & 2.4542 & 0.0143 \\
PC 4 & 0.0216 & 0.0938 & 0.2305 & 0.8178 \\
PC 5 & 0.1625 & 0.1117 & 1.4540 & 0.1462 \\
PC 6 & 0.1163 & 0.1027 & 1.1326 & 0.2576 \\
PC 7 & 0.1787 & 0.1040 & 1.7185 & 0.0859 \\
PC 8 & 0.3199 & 0.2611 & 1.2255 & 0.2206 \\
\hline B. smooth terms & edf & Ref.df & F-value & p-value \\
Location Smooth & 89.4823 & 245.0000 & 1.7630 & $<0.0001$ \\
Year Smooth & 1.8783 & 19.0000 & 26.3486 & $<0.0001$ \\
Station Random Effect & 4.2974 & 7.0000 & 24.5457 & $<0.0001$ \\
\hline
\end{tabular}

Table A3.123: Best productivity model for the White-eyed Vireo (Vireo griseus). The model was a quasi-Poisson GAMM with log link function. Based on AIC/QIC, support for including the noise level as a predictor of productivity was: strong; support for including the standard deviation of noise was: strong. 


\begin{tabular}{lrrrr}
\hline A. parametric coefficients & Estimate & Std. Error & t-value & p-value \\
Intercept & -6.5775 & 1.6463 & -3.9953 & 0.0001 \\
Noise Level & 0.0269 & 0.0410 & 0.6566 & 0.5115 \\
PC 1 & -0.3005 & 0.1588 & -1.8931 & 0.0585 \\
PC 2 & 0.1389 & 0.1279 & 1.0862 & 0.2775 \\
PC 3 & -0.3465 & 0.1818 & -1.9059 & 0.0568 \\
PC 4 & 0.0137 & 0.1521 & 0.0902 & 0.9281 \\
PC 5 & -0.0039 & 0.1817 & -0.0217 & 0.9827 \\
PC 6 & 0.2126 & 0.1705 & 1.2463 & 0.2128 \\
PC 7 & -0.3512 & 0.1620 & -2.1678 & 0.0303 \\
PC 8 & -0.1194 & 0.2414 & -0.4945 & 0.6210 \\
\hline B. smooth terms & edf & Ref.df & F-value & p-value \\
Location Smooth & 4.4890 & 19.0000 & 3081.1293 & $<0.0001$ \\
Year Smooth & 4.5324 & 7.0000 & 108.4404 & $<0.0001$ \\
Station Random Effect & 209.9784 & 258.0000 & 7.6952 & $<0.0001$ \\
\hline
\end{tabular}

Table A3.124: Best abundance model for the White-eyed Vireo (Vireo griseus). The model was a quasi-Poisson GAMM with log link function. Based on AIC/QIC, support for including the noise level as a predictor of abundance was: strong; support for including the standard deviation of noise was: none.

\begin{tabular}{lrrrr}
\hline A. parametric coefficients & Estimate & Std. Error & t-value & p-value \\
Intercept & -1.8035 & 0.2136 & -8.4439 & $<0.0001$ \\
Noise Standard Deviation & -0.1473 & 0.1901 & -0.7748 & 0.4386 \\
PC 1 & -0.4171 & 0.1076 & -3.8766 & 0.0001 \\
PC 2 & -0.0583 & 0.1136 & -0.5133 & 0.6078 \\
PC 3 & -0.3685 & 0.1338 & -2.7547 & 0.0059 \\
PC 4 & 0.0092 & 0.1169 & 0.0785 & 0.9374 \\
PC 5 & 0.0215 & 0.1488 & 0.1444 & 0.8852 \\
PC 6 & 0.2193 & 0.1523 & 1.4399 & 0.1501 \\
PC 7 & 0.1533 & 0.1618 & 0.9469 & 0.3438 \\
PC 8 & -0.9138 & 0.2849 & -3.2072 & 0.0014 \\
\hline B. smooth terms & edf & Ref.df & F-value & p-value \\
Location Smooth & 113.8732 & 235.0000 & 3.5792 & $<0.0001$ \\
Year Smooth & 8.7288 & 19.0000 & 391.9643 & 0.0003 \\
Station Random Effect & 1.4231 & 7.0000 & 6.8090 & 0.0255 \\
\hline
\end{tabular}

Table A3.125: Best productivity model for the Wilson's Warbler (Cardellina pusilla). The model was a quasi-Poisson GAMM with log link function. Based on AIC/QIC, support for including the noise level as a predictor of productivity was: none; support for including the standard deviation of noise was: weak. 


\begin{tabular}{lrrrr}
\hline A. parametric coefficients & Estimate & Std. Error & t-value & p-value \\
Intercept & -3.7064 & 1.5457 & -2.3979 & 0.0166 \\
Noise Level & -0.0408 & 0.0438 & -0.9326 & 0.3511 \\
Noise Standard Deviation & 0.2853 & 0.1823 & 1.5653 & 0.1177 \\
PC 1 & 0.1302 & 0.1399 & 0.9307 & 0.3521 \\
PC 2 & 0.0684 & 0.0969 & 0.7062 & 0.4801 \\
PC 3 & -0.0494 & 0.1055 & -0.4680 & 0.6398 \\
PC 4 & -0.0504 & 0.0969 & -0.5201 & 0.6030 \\
PC 5 & -0.0002 & 0.1159 & -0.0021 & 0.9983 \\
PC 6 & -0.2077 & 0.1178 & -1.7634 & 0.0780 \\
PC 7 & -0.0182 & 0.1396 & -0.1302 & 0.8964 \\
PC 8 & -0.1653 & 0.2683 & -0.6161 & 0.5379 \\
\hline B. smooth terms & edf & Ref.df & F-value & p-value \\
Location Smooth & 12.0896 & 19.0000 & 2066.2913 & 0.0015 \\
Year Smooth & 4.6708 & 7.0000 & 41.6297 & $<0.0001$ \\
Station Random Effect & 181.7727 & 238.0000 & 9.3733 & $<0.0001$ \\
\hline
\end{tabular}

Table A3.126: Best abundance model for the Wilson's Warbler (Cardellina pusilla). The model was a quasi-Poisson GAMM with log link function. Based on AIC/QIC, support for including the noise level as a predictor of abundance was: weak; support for including the standard deviation of noise was: weak.

\begin{tabular}{lrrrr}
\hline A. parametric coefficients & Estimate & Std. Error & t-value & p-value \\
Intercept & -3.1867 & 1.1024 & -2.8908 & 0.0039 \\
Noise Level & 0.0260 & 0.0269 & 0.9668 & 0.3338 \\
PC 1 & 0.0128 & 0.0918 & 0.1390 & 0.8895 \\
PC 2 & -0.1177 & 0.1007 & -1.1683 & 0.2428 \\
PC 3 & -0.4947 & 0.2954 & -1.6750 & 0.0941 \\
PC 4 & -0.1151 & 0.1465 & -0.7857 & 0.4322 \\
PC 5 & 0.0631 & 0.1682 & 0.3751 & 0.7076 \\
PC 6 & -0.0404 & 0.2891 & -0.1398 & 0.8888 \\
PC 7 & 0.1082 & 0.1346 & 0.8036 & 0.4218 \\
PC 8 & 0.0162 & 0.0868 & 0.1865 & 0.8521 \\
\hline B. smooth terms & edf & Ref.df & F-value & p-value \\
Location Smooth & 143.3546 & 322.0000 & 2.2492 & $<0.0001$ \\
Year Smooth & 0.0013 & 19.0000 & 0.0001 & 0.4622 \\
Station Random Effect & 0.0020 & 7.0000 & 0.0001 & 0.6323 \\
\hline
\end{tabular}

Table A3.127: Best productivity model for the Wood Thrush (Hylocichla mustelina). The model was a quasi-Poisson GAMM with log link function. Based on AIC/QIC, support for including the noise level as a predictor of productivity was: strong; support for including the standard deviation of noise was: none. 


\begin{tabular}{lrrrr}
\hline A. parametric coefficients & Estimate & Std. Error & t-value & p-value \\
Intercept & -7.3228 & 1.4044 & -5.2141 & $<0.0001$ \\
Noise Level & 0.0597 & 0.0345 & 1.7278 & 0.0841 \\
PC 1 & -0.1518 & 0.1171 & -1.2963 & 0.1950 \\
PC 2 & -0.1316 & 0.1321 & -0.9963 & 0.3192 \\
PC 3 & 0.2008 & 0.3731 & 0.5383 & 0.5904 \\
PC 4 & -0.1823 & 0.1580 & -1.1534 & 0.2489 \\
PC 5 & 0.0382 & 0.1769 & 0.2157 & 0.8293 \\
PC 6 & -0.7247 & 0.3248 & -2.2313 & 0.0258 \\
PC 7 & 0.2429 & 0.1638 & 1.4824 & 0.1384 \\
PC 8 & 0.0002 & 0.1086 & 0.0021 & 0.9983 \\
\hline B. smooth terms & edf & Ref.df & F-value & p-value \\
Location Smooth & 11.8349 & 19.0000 & 1938.3552 & $<0.0001$ \\
Year Smooth & 4.1841 & 7.0000 & 90.5011 & 0.0003 \\
Station Random Effect & 260.8676 & 331.0000 & 9.9771 & $<0.0001$ \\
\hline
\end{tabular}

Table A3.128: Best abundance model for the Wood Thrush (Hylocichla mustelina). The model was a quasi-Poisson GAMM with log link function. Based on AIC/QIC, support for including the noise level as a predictor of abundance was: strong; support for including the standard deviation of noise was: none.

\begin{tabular}{lrrrr}
\hline A. parametric coefficients & Estimate & Std. Error & t-value & p-value \\
Intercept & -0.1044 & 0.1503 & -0.6946 & 0.4876 \\
Noise Standard Deviation & -0.1149 & 0.1236 & -0.9297 & 0.3529 \\
PC 1 & -0.0852 & 0.0578 & -1.4742 & 0.1409 \\
PC 2 & -0.0494 & 0.0634 & -0.7790 & 0.4363 \\
PC 3 & -0.0961 & 0.0759 & -1.2661 & 0.2059 \\
PC 4 & 0.1484 & 0.0829 & 1.7898 & 0.0740 \\
PC 5 & 0.0706 & 0.0892 & 0.7916 & 0.4289 \\
PC 6 & -0.1442 & 0.0798 & -1.8071 & 0.0712 \\
PC 7 & 0.1393 & 0.0702 & 1.9823 & 0.0479 \\
PC 8 & 0.0633 & 0.1291 & 0.4905 & 0.6239 \\
\hline B. smooth terms & edf & Ref.df & F-value & p-value \\
Location Smooth & 26.7173 & 104.0000 & 0.7804 & $<0.0001$ \\
Year Smooth & 2.8340 & 19.0000 & 2.4774 & 0.0009 \\
Station Random Effect & 3.4833 & 7.0000 & 2.4644 & 0.0400 \\
\hline
\end{tabular}

Table A3.129: Best productivity model for the Wrentit (Chamaea fasciata). The model was a quasi-Poisson GAMM with log link function. Based on AIC/QIC, support for including the noise level as a predictor of productivity was: none; support for including the standard deviation of noise was: weak. 


\begin{tabular}{lrrrr}
\hline A. parametric coefficients & Estimate & Std. Error & t-value & p-value \\
Intercept & -5.3753 & 0.1684 & -31.9123 & $<0.0001$ \\
PC 1 & 0.4008 & 0.1028 & 3.8996 & 0.0001 \\
PC 2 & 0.3993 & 0.1236 & 3.2310 & 0.0013 \\
PC 3 & 0.2884 & 0.1063 & 2.7132 & 0.0068 \\
PC 4 & 0.5443 & 0.1356 & 4.0156 & 0.0001 \\
PC 5 & 0.3265 & 0.1552 & 2.1035 & 0.0357 \\
PC 6 & -0.4395 & 0.1292 & -3.4009 & 0.0007 \\
PC 7 & -0.1766 & 0.1571 & -1.1243 & 0.2612 \\
PC 8 & -0.3247 & 0.2822 & -1.1506 & 0.2502 \\
\hline B. smooth terms & edf & Ref.df & F-value & p-value \\
Location Smooth & 0.0004 & 19.0000 & 0.0000 & 0.9437 \\
Year Smooth & 1.0409 & 7.0000 & 21.8140 & 0.0317 \\
Station Random Effect & 93.3017 & 113.0000 & 10.8136 & $<0.0001$ \\
\hline
\end{tabular}

Table A3.130: Best abundance model for the Wrentit (Chamaea fasciata). The model was a quasi-Poisson GAMM with log link function. Based on AIC/QIC, support for including the noise level as a predictor of abundance was: none; support for including the standard deviation of noise was: none.

\begin{tabular}{lrrrr}
\hline A. parametric coefficients & Estimate & Std. Error & t-value & p-value \\
Intercept & -2.1225 & 0.6255 & -3.3934 & 0.0009 \\
Noise Standard Deviation & 0.3182 & 0.4379 & 0.7268 & 0.4684 \\
PC 1 & -0.0500 & 0.1728 & -0.2891 & 0.7729 \\
PC 2 & -0.0337 & 0.2191 & -0.1539 & 0.8779 \\
PC 3 & -0.5025 & 0.6382 & -0.7874 & 0.4322 \\
PC 4 & 0.1204 & 0.3509 & 0.3430 & 0.7320 \\
PC 5 & -0.0525 & 0.4231 & -0.1242 & 0.9013 \\
PC 6 & 0.3984 & 0.6658 & 0.5983 & 0.5504 \\
PC 7 & -0.0998 & 0.2342 & -0.4263 & 0.6705 \\
PC 8 & 0.0618 & 0.3703 & 0.1669 & 0.8677 \\
\hline B. smooth terms & edf & Ref.df & F-value & p-value \\
Location Smooth & 7.0137 & 40.0000 & 0.3427 & 0.0047 \\
Year Smooth & 2.7148 & 19.0000 & 0.6054 & 0.1354 \\
Station Random Effect & 0.0002 & 7.0000 & 0.0000 & 0.4998 \\
\hline
\end{tabular}

Table A3.131: Best productivity model for the White-throated Sparrow (Zonotrichia albicollis). The model was a quasi-Poisson GAMM with log link function. Based on AIC/QIC, support for including the noise level as a predictor of productivity was: none; support for including the standard deviation of noise was: weak. 


\begin{tabular}{lrrrr}
\hline A. parametric coefficients & Estimate & Std. Error & t-value & p-value \\
Intercept & -3.4544 & 1.0421 & -3.3147 & 0.0010 \\
Noise Standard Deviation & -1.9385 & 0.7727 & -2.5088 & 0.0127 \\
PC 1 & 0.0998 & 0.2513 & 0.3973 & 0.6914 \\
PC 2 & -0.0043 & 0.3488 & -0.0125 & 0.9901 \\
PC 3 & 0.7942 & 0.9063 & 0.8763 & 0.3816 \\
PC 4 & -0.6124 & 0.5344 & -1.1461 & 0.2527 \\
PC 5 & 1.8959 & 0.6362 & 2.9801 & 0.0031 \\
PC 6 & 1.9252 & 1.1532 & 1.6695 & 0.0961 \\
PC 7 & -0.2542 & 0.3575 & -0.7110 & 0.4776 \\
PC 8 & 0.2279 & 0.3949 & 0.5770 & 0.5644 \\
\hline B. smooth terms & edf & Ref.df & F-value & p-value \\
Location Smooth & 1.7211 & 19.0000 & 207.5074 & 0.0245 \\
Year Smooth & 5.8443 & 7.0000 & 81.1486 & $<0.0001$ \\
Station Random Effect & 36.0342 & 46.0000 & 8.3351 & $<0.0001$ \\
\hline
\end{tabular}

Table A3.132: Best abundance model for the White-throated Sparrow (Zonotrichia albicollis). The model was a quasi-Poisson GAMM with log link function. Based on AIC/QIC, support for including the noise level as a predictor of abundance was: none; support for including the standard deviation of noise was: weak.

\begin{tabular}{lrrrr}
\hline A. parametric coefficients & Estimate & Std. Error & t-value & p-value \\
Intercept & -2.2308 & 0.1059 & -21.0628 & $<0.0001$ \\
PC 1 & -0.3164 & 0.1545 & -2.0487 & 0.0407 \\
PC 2 & 0.1598 & 0.1121 & 1.4258 & 0.1542 \\
PC 3 & -0.1941 & 0.0868 & -2.2361 & 0.0255 \\
PC 4 & 0.0325 & 0.0839 & 0.3875 & 0.6985 \\
PC 5 & 0.0634 & 0.0666 & 0.9522 & 0.3412 \\
PC 6 & 0.0271 & 0.0911 & 0.2974 & 0.7662 \\
PC 7 & -0.0584 & 0.0945 & -0.6179 & 0.5367 \\
PC 8 & -0.2770 & 0.2826 & -0.9804 & 0.3271 \\
\hline B. smooth terms & edf & Ref.df & F-value & p-value \\
Location Smooth & 88.4922 & 304.0000 & 0.6415 & $<0.0001$ \\
Year Smooth & 4.9473 & 19.0000 & 76.2308 & $<0.0001$ \\
Station Random Effect & 4.7569 & 7.0000 & 8.9024 & 0.0001 \\
\hline
\end{tabular}

Table A3.133: Best productivity model for the Yellow-breasted Chat (Icteria virens). The model was a quasi-Poisson GAMM with log link function. Based on AIC/QIC, support for including the noise level as a predictor of productivity was: none; support for including the standard deviation of noise was: none. 


\begin{tabular}{lrrrr}
\hline A. parametric coefficients & Estimate & Std. Error & t-value & p-value \\
Intercept & -5.2072 & 1.7948 & -2.9013 & 0.0038 \\
Noise Level & -0.0214 & 0.0479 & -0.4463 & 0.6554 \\
Noise Standard Deviation & 0.3429 & 0.2765 & 1.2402 & 0.2150 \\
PC 1 & -0.1331 & 0.1730 & -0.7693 & 0.4418 \\
PC 2 & 0.1221 & 0.1322 & 0.9237 & 0.3557 \\
PC 3 & 0.1821 & 0.1281 & 1.4218 & 0.1552 \\
PC 4 & -0.0153 & 0.1125 & -0.1356 & 0.8921 \\
PC 5 & 0.0291 & 0.1089 & 0.2669 & 0.7896 \\
PC 6 & 0.0840 & 0.1293 & 0.6498 & 0.5159 \\
PC 7 & 0.0205 & 0.1339 & 0.1529 & 0.8785 \\
PC 8 & -0.3616 & 0.3292 & -1.0983 & 0.2722 \\
\hline B. smooth terms & edf & Ref.df & F-value & p-value \\
Location Smooth & 12.6001 & 19.0000 & 15420.6455 & 0.0002 \\
Year Smooth & 3.8755 & 7.0000 & 1245.6819 & $<0.0001$ \\
Station Random Effect & 258.1030 & 311.0000 & 16.0151 & $<0.0001$ \\
\hline
\end{tabular}

Table A3.134: Best abundance model for the Yellow-breasted Chat (Icteria virens). The model was a quasi-Poisson GAMM with log link function. Based on AIC/QIC, support for including the noise level as a predictor of abundance was: weak; support for including the standard deviation of noise was: strong.

\begin{tabular}{lrrrr}
\hline A. parametric coefficients & Estimate & Std. Error & t-value & p-value \\
Intercept & -15.2637 & 16.4868 & -0.9258 & 0.3550 \\
Noise Level & 0.2373 & 0.4018 & 0.5905 & 0.5551 \\
PC 1 & -0.4568 & 2.1180 & -0.2157 & 0.8293 \\
PC 2 & 0.4461 & 1.6418 & 0.2717 & 0.7860 \\
PC 3 & 0.6672 & 0.7684 & 0.8682 & 0.3857 \\
PC 4 & -0.7352 & 2.6011 & -0.2827 & 0.7776 \\
PC 5 & -0.0844 & 2.8470 & -0.0296 & 0.9764 \\
PC 6 & -0.4196 & 1.6551 & -0.2535 & 0.8000 \\
PC 7 & 0.2495 & 2.3231 & 0.1074 & 0.9145 \\
PC 8 & -1.3928 & 2.9762 & -0.4680 & 0.6400 \\
\hline B. smooth terms & edf & Ref.df & F-value & p-value \\
Location Smooth & 48.1320 & 198.0000 & 0.1652 & 0.9898 \\
Year Smooth & 0.0001 & 19.0000 & 0.0000 & 1.0000 \\
Station Random Effect & 4.9260 & 7.0000 & 13.5999 & 0.2927 \\
\hline
\end{tabular}

Table A3.135: Best productivity model for the Yellow-billed Cuckoo (Coccyzus americanus). The model was a quasi-Poisson GAMM with log link function. Based on AIC/QIC, support for including the noise level as a predictor of productivity was: strong; support for including the standard deviation of noise was: none. 


\begin{tabular}{lrrrr}
\hline A. parametric coefficients & Estimate & Std. Error & t-value & p-value \\
Intercept & -7.2793 & 0.2024 & -35.9673 & $<0.0001$ \\
Noise Standard Deviation & 0.0747 & 0.2300 & 0.3246 & 0.7455 \\
PC 1 & 0.0565 & 0.0916 & 0.6172 & 0.5372 \\
PC 2 & 0.2505 & 0.0940 & 2.6656 & 0.0078 \\
PC 3 & 0.2176 & 0.1086 & 2.0042 & 0.0452 \\
PC 4 & -0.1537 & 0.1299 & -1.1831 & 0.2369 \\
PC 5 & -0.0547 & 0.1393 & -0.3923 & 0.6949 \\
PC 6 & -0.0120 & 0.1025 & -0.1169 & 0.9069 \\
PC 7 & 0.0338 & 0.1313 & 0.2575 & 0.7968 \\
PC 8 & 0.0054 & 0.1390 & 0.0390 & 0.9689 \\
\hline B. smooth terms & edf & Ref.df & F-value & p-value \\
Location Smooth & 6.5639 & 19.0000 & 30.9373 & 0.0007 \\
Year Smooth & 2.4364 & 7.0000 & 4.8120 & 0.0063 \\
Station Random Effect & 121.8483 & 207.0000 & 2.0653 & $<0.0001$ \\
\hline
\end{tabular}

Table A3.136: Best abundance model for the Yellow-billed Cuckoo (Coccyzus americanus). The model was a quasi-Poisson GAMM with log link function. Based on AIC/QIC, support for including the noise level as a predictor of abundance was: none; support for including the standard deviation of noise was: weak. 


\begin{tabular}{lrrrr}
\hline A. parametric coefficients & Estimate & Std. Error & t-value & p-value \\
Intercept & -1.6743 & 0.0672 & -24.9337 & $<0.0001$ \\
PC 1 & -0.0733 & 0.0588 & -1.2468 & 0.2126 \\
PC 2 & 0.0148 & 0.0565 & 0.2619 & 0.7934 \\
PC 3 & 0.0658 & 0.0554 & 1.1887 & 0.2347 \\
PC 4 & -0.0084 & 0.0487 & -0.1718 & 0.8636 \\
PC 5 & 0.0030 & 0.0566 & 0.0536 & 0.9572 \\
PC 6 & -0.1000 & 0.0654 & -1.5285 & 0.1265 \\
PC 7 & 0.1793 & 0.0516 & 3.4784 & 0.0005 \\
PC 8 & -0.0189 & 0.1380 & -0.1370 & 0.8910 \\
\hline B. smooth terms & edf & Ref.df & F-value & p-value \\
Location Smooth & 136.5441 & 402.0000 & 1.5130 & $<0.0001$ \\
Year Smooth & 10.0492 & 19.0000 & 61.5396 & 0.0369 \\
Station Random Effect & 5.5344 & 7.0000 & 25.8853 & $<0.0001$ \\
\hline
\end{tabular}

Table A3.137: Best productivity model for the Yellow Warbler (Setophaga petechia). The model was a quasi-Poisson GAMM with log link function. Based on AIC/QIC, support for including the noise level as a predictor of productivity was: none; support for including the standard deviation of noise was: none. 


\begin{tabular}{lrrrr}
\hline A. parametric coefficients & Estimate & Std. Error & t-value & p-value \\
Intercept & -6.5718 & 1.3732 & -4.7856 & $<0.0001$ \\
Noise Level & 0.0369 & 0.0375 & 0.9834 & 0.3255 \\
PC 1 & 0.1892 & 0.1192 & 1.5875 & 0.1125 \\
PC 2 & 0.2634 & 0.0917 & 2.8739 & 0.0041 \\
PC 3 & 0.0984 & 0.0914 & 1.0766 & 0.2817 \\
PC 4 & -0.1026 & 0.0765 & -1.3412 & 0.1800 \\
PC 5 & -0.1027 & 0.0881 & -1.1654 & 0.2439 \\
PC 6 & -0.0613 & 0.0962 & -0.6374 & 0.5239 \\
PC 7 & 0.1399 & 0.0886 & 1.5791 & 0.1144 \\
PC 8 & -0.0164 & 0.2064 & -0.0793 & 0.9368 \\
\hline B. smooth terms & edf & Ref.df & F-value & p-value \\
Location Smooth & 13.2391 & 19.0000 & 34498.6745 & $<0.0001$ \\
Year Smooth & 6.0438 & 7.0000 & 911.2041 & $<0.0001$ \\
Station Random Effect & 344.2253 & 417.0000 & 13.5456 & $<0.0001$ \\
\hline
\end{tabular}

Table A3.138: Best abundance model for the Yellow Warbler (Setophaga petechia). The model was a quasi-Poisson GAMM with log link function. Based on AIC/QIC, support for including the noise level as a predictor of abundance was: weak; support for including the standard deviation of noise was: none. 


\section{Appendix 4: Model selection tables for individual bird species}

Note: Models for the Golden-cheeked Warbler (Setophaga chrysoparia) and Tennessee Warbler (Leiothlypis peregrina) could not be fitted, as stated in the main text.

\section{Abundance Models}

\begin{tabular}{lrrr}
\hline name & QIC & delta QIC & Akaike weight \\
\hline No Noise Terms & 5458.77 & 0.00 & 0.59 \\
No Mean Noise Term & 5460.58 & 1.82 & 0.24 \\
No Noise Variability Term & 5462.05 & 3.29 & 0.11 \\
Full Model & 5463.23 & 4.47 & 0.06 \\
\hline
\end{tabular}

Table A4.1: Model selection information for the abundance model for the Acadian Flycatcher (Empidonax virescens). The model was a quasi-Poisson GAMM with log link function. Based on QIC, support for including the noise level as a predictor of abundance was: none; support for including the standard deviation of noise was: none.

\begin{tabular}{lrrr}
\hline name & QIC & delta QIC & Akaike weight \\
\hline Full Model & 6493.34 & 0.00 & 0.43 \\
No Noise Variability Term & 6494.32 & 0.98 & 0.26 \\
No Mean Noise Term & 6495.03 & 1.70 & 0.18 \\
No Noise Terms & 6495.82 & 2.48 & 0.12 \\
\hline
\end{tabular}

Table A4.2: Model selection information for the abundance model for the American Goldfinch (Spinus tristis). The model was a quasi-Poisson GAMM with log link function. Based on QIC, support for including the noise level as a predictor of abundance was: weak; support for including the standard deviation of noise was: weak. 


\begin{tabular}{lrrr}
\hline name & QIC & delta QIC & Akaike weight \\
\hline Full Model & 4151.80 & 0.00 & 0.59 \\
No Mean Noise Term & 4153.19 & 1.39 & 0.30 \\
No Noise Variability Term & 4156.10 & 4.30 & 0.07 \\
No Noise Terms & 4157.02 & 5.23 & 0.04 \\
\hline
\end{tabular}

Table A4.3: Model selection information for the abundance model for the American Redstart (Setophaga ruticilla). The model was a quasi-Poisson GAMM with log link function. Based on QIC, support for including the noise level as a predictor of abundance was: weak; support for including the standard deviation of noise was: strong.

\begin{tabular}{lrrr}
\hline name & QIC & delta QIC & Akaike weight \\
\hline No Noise Variability Term & 11975.09 & 0.00 & 0.29 \\
Full Model & 11975.23 & 0.14 & 0.27 \\
No Noise Terms & 11975.53 & 0.44 & 0.23 \\
No Mean Noise Term & 11975.75 & 0.66 & 0.21 \\
\hline
\end{tabular}

Table A4.4: Model selection information for the abundance model for the American Robin (Turdus migratorius). The model was a quasi-Poisson GAMM with log link function. Based on QIC, support for including the noise level as a predictor of abundance was: weak; support for including the standard deviation of noise was: none.

\begin{tabular}{lrrr}
\hline name & QIC & delta QIC & Akaike weight \\
\hline No Mean Noise Term & 2209.29 & 0.00 & 0.50 \\
Full Model & 2210.47 & 1.18 & 0.28 \\
No Noise Terms & 2211.78 & 2.48 & 0.15 \\
No Noise Variability Term & 2213.12 & 3.83 & 0.07 \\
\hline
\end{tabular}

Table A4.5: Model selection information for the abundance model for the Ash-throated Flycatcher (Myiarchus cinerascens). The model was a quasi-Poisson GAMM with log link function. Based on QIC, support for including the noise level as a predictor of abundance was: none; support for including the standard deviation of noise was: strong.

\begin{tabular}{lrrr}
\hline name & QIC & delta QIC & Akaike weight \\
\hline No Mean Noise Term & 4294.39 & 0.00 & 0.38 \\
Full Model & 4295.36 & 0.97 & 0.23 \\
No Noise Terms & 4295.69 & 1.29 & 0.20 \\
No Noise Variability Term & 4295.82 & 1.43 & 0.19 \\
\hline
\end{tabular}

Table A4.6: Model selection information for the abundance model for the Audubon's Warbler (Setophaga coronata auduboni). The model was a quasi-Poisson GAMM with log link function. Based on QIC, support for including the noise level as a predictor of abundance was: none; support for including the standard deviation of noise was: weak. 


\begin{tabular}{lrrr}
\hline name & QIC & delta QIC & Akaike weight \\
\hline No Noise Variability Term & 6287.74 & 0.00 & 0.48 \\
Full Model & 6289.14 & 1.40 & 0.24 \\
No Noise Terms & 6289.70 & 1.96 & 0.18 \\
No Mean Noise Term & 6290.68 & 2.94 & 0.11 \\
\hline
\end{tabular}

Table A4.7: Model selection information for the abundance model for the Black-capped Chickadee (Poecile atricapillus). The model was a quasi-Poisson GAMM with log link function. Based on QIC, support for including the noise level as a predictor of abundance was: weak; support for including the standard deviation of noise was: none.

\begin{tabular}{lrrr}
\hline name & QIC & delta QIC & Akaike weight \\
\hline No Noise Variability Term & 2048.61 & 0.00 & 0.29 \\
No Noise Terms & 2048.79 & 0.19 & 0.27 \\
Full Model & 2049.00 & 0.40 & 0.24 \\
No Mean Noise Term & 2049.30 & 0.69 & 0.21 \\
\hline
\end{tabular}

Table A4.8: Model selection information for the abundance model for the Black-chinned Hummingbird (Archilochus alexandri). The model was a quasi-Poisson GAMM with log link function. Based on QIC, support for including the noise level as a predictor of abundance was: weak; support for including the standard deviation of noise was: none.

\begin{tabular}{lrrr}
\hline name & QIC & delta QIC & Akaike weight \\
\hline No Noise Variability Term & 4467.89 & 0.00 & 0.28 \\
No Noise Terms & 4468.10 & 0.20 & 0.25 \\
Full Model & 4468.17 & 0.28 & 0.24 \\
No Mean Noise Term & 4468.22 & 0.33 & 0.23 \\
\hline
\end{tabular}

Table A4.9: Model selection information for the abundance model for the Bewick's Wren (Thryomanes bewickii). The model was a quasi-Poisson GAMM with log link function. Based on QIC, support for including the noise level as a predictor of abundance was: weak; support for including the standard deviation of noise was: none.

\begin{tabular}{lrrr}
\hline name & QIC & delta QIC & Akaike weight \\
\hline No Mean Noise Term & 6231.16 & 0.00 & 0.41 \\
Full Model & 6232.30 & 1.14 & 0.23 \\
No Noise Terms & 6232.38 & 1.22 & 0.22 \\
No Noise Variability Term & 6233.34 & 2.18 & 0.14 \\
\hline
\end{tabular}

Table A4.10: Model selection information for the abundance model for the Black-headed Grosbeak (Pheucticus melanocephalus). The model was a quasi-Poisson GAMM with log link function. Based on QIC, support for including the noise level as a predictor of abundance was: none; support for including the standard deviation of noise was: weak. 


\begin{tabular}{lrrr}
\hline name & QIC & delta QIC & Akaike weight \\
\hline No Noise Variability Term & 229.20 & 0.00 & 0.38 \\
No Noise Terms & 230.16 & 0.96 & 0.24 \\
Full Model & 230.49 & 1.29 & 0.20 \\
No Mean Noise Term & 230.76 & 1.56 & 0.18 \\
\hline
\end{tabular}

Table A4.11: Model selection information for the abundance model for the Bobolink (Dolichonyx oryzivorus). The model was a quasi-Poisson GAMM with log link function. Based on QIC, support for including the noise level as a predictor of abundance was: weak; support for including the standard deviation of noise was: none.

\begin{tabular}{lrrr}
\hline name & QIC & delta QIC & Akaike weight \\
\hline Full Model & 2371.25 & 0.00 & 0.29 \\
No Mean Noise Term & 2371.31 & 0.06 & 0.28 \\
No Noise Terms & 2371.80 & 0.55 & 0.22 \\
No Noise Variability Term & 2371.96 & 0.71 & 0.20 \\
\hline
\end{tabular}

Table A4.12: Model selection information for the abundance model for the Bushtit (Psaltriparus minimus). The model was a quasi-Poisson GAMM with log link function. Based on QIC, support for including the noise level as a predictor of abundance was: weak; support for including the standard deviation of noise was: weak.

\begin{tabular}{lrrr}
\hline name & QIC & delta QIC & Akaike weight \\
\hline No Noise Terms & 1712.41 & 0.00 & 0.31 \\
No Mean Noise Term & 1712.63 & 0.22 & 0.28 \\
No Noise Variability Term & 1713.02 & 0.61 & 0.23 \\
Full Model & 1713.47 & 1.06 & 0.18 \\
\hline
\end{tabular}

Table A4.13: Model selection information for the abundance model for the Cassin's Finch (Haemorhous cassinii). The model was a quasi-Poisson GAMM with log link function. Based on QIC, support for including the noise level as a predictor of abundance was: none; support for including the standard deviation of noise was: none. 


\begin{tabular}{lrrr}
\hline name & QIC & delta QIC & Akaike weight \\
\hline No Noise Variability Term & 6604.88 & 0.00 & 0.35 \\
Full Model & 6605.01 & 0.13 & 0.33 \\
No Mean Noise Term & 6606.38 & 1.50 & 0.16 \\
No Noise Terms & 6606.43 & 1.55 & 0.16 \\
\hline
\end{tabular}

Table A4.14: Model selection information for the abundance model for the Carolina Wren (Thryothorus ludovicianus). The model was a quasi-Poisson GAMM with log link function. Based on QIC, support for including the noise level as a predictor of abundance was: weak; support for including the standard deviation of noise was: none.

\begin{tabular}{lrrr}
\hline name & QIC & delta QIC & Akaike weight \\
\hline No Noise Variability Term & 3114.19 & 0.00 & 0.68 \\
Full Model & 3116.35 & 2.15 & 0.23 \\
No Noise Terms & 3118.97 & 4.77 & 0.06 \\
No Mean Noise Term & 3121.06 & 6.86 & 0.02 \\
\hline
\end{tabular}

Table A4.15: Model selection information for the abundance model for the Cassin's Vireo (Vireo cassinii). The model was a quasi-Poisson GAMM with log link function. Based on QIC, support for including the noise level as a predictor of abundance was: strong; support for including the standard deviation of noise was: none.

\begin{tabular}{lrrr}
\hline name & QIC & delta QIC & Akaike weight \\
\hline Full Model & 4027.51 & 0.00 & 0.28 \\
No Noise Variability Term & 4027.63 & 0.12 & 0.26 \\
No Mean Noise Term & 4027.76 & 0.25 & 0.25 \\
No Noise Terms & 4028.05 & 0.54 & 0.21 \\
\hline
\end{tabular}

Table A4.16: Model selection information for the abundance model for the Cedar Waxwing (Bombycilla cedrorum). The model was a quasi-Poisson GAMM with log link function. Based on QIC, support for including the noise level as a predictor of abundance was: weak; support for including the standard deviation of noise was: weak.

\begin{tabular}{lrrr}
\hline name & QIC & delta QIC & Akaike weight \\
\hline Full Model & 4953.61 & 0.00 & 0.41 \\
No Mean Noise Term & 4954.46 & 0.85 & 0.27 \\
No Noise Terms & 4954.98 & 1.37 & 0.20 \\
No Noise Variability Term & 4956.00 & 2.39 & 0.12 \\
\hline
\end{tabular}

Table A4.17: Model selection information for the abundance model for the Chipping Sparrow (Spizella passerina). The model was a quasi-Poisson GAMM with log link function. Based on QIC, support for including the noise level as a predictor of abundance was: weak; support for including the standard deviation of noise was: weak. 


\begin{tabular}{lrrr}
\hline name & QIC & delta QIC & Akaike weight \\
\hline No Noise Variability Term & 9302.44 & 0.00 & 0.29 \\
Full Model & 9302.66 & 0.22 & 0.26 \\
No Noise Terms & 9302.90 & 0.46 & 0.23 \\
No Mean Noise Term & 9303.09 & 0.66 & 0.21 \\
\hline
\end{tabular}

Table A4.18: Model selection information for the abundance model for the Common Yellowthroat (Geothlypis trichas). The model was a quasi-Poisson GAMM with log link function. Based on QIC, support for including the noise level as a predictor of abundance was: weak; support for including the standard deviation of noise was: none.

\begin{tabular}{lrrr}
\hline name & QIC & delta QIC & Akaike weight \\
\hline No Noise Terms & 4574.84 & 0.00 & 0.44 \\
No Mean Noise Term & 4575.63 & 0.79 & 0.30 \\
Full Model & 4577.20 & 2.36 & 0.14 \\
No Noise Variability Term & 4577.47 & 2.63 & 0.12 \\
\hline
\end{tabular}

Table A4.19: Model selection information for the abundance model for the Dark-eyed Junco (Junco hyemalis). The model was a quasi-Poisson GAMM with log link function. Based on QIC, support for including the noise level as a predictor of abundance was: none; support for including the standard deviation of noise was: none.

\begin{tabular}{lrrr}
\hline name & QIC & delta QIC & Akaike weight \\
\hline No Noise Variability Term & 3310.22 & 0.00 & 0.35 \\
Full Model & 3310.47 & 0.26 & 0.30 \\
No Noise Terms & 3311.36 & 1.14 & 0.20 \\
No Mean Noise Term & 3311.81 & 1.60 & 0.16 \\
\hline
\end{tabular}

Table A4.20: Model selection information for the abundance model for the Dusky Flycatcher (Empidonax oberholseri). The model was a quasi-Poisson GAMM with log link function. Based on QIC, support for including the noise level as a predictor of abundance was: weak; support for including the standard deviation of noise was: none. 


\begin{tabular}{lrrr}
\hline name & QIC & delta QIC & Akaike weight \\
\hline No Mean Noise Term & 1929.70 & 0.00 & 0.54 \\
Full Model & 1930.13 & 0.43 & 0.43 \\
No Noise Terms & 1936.51 & 6.81 & 0.02 \\
No Noise Variability Term & 1936.92 & 7.22 & 0.01 \\
\hline
\end{tabular}

Table A4.21: Model selection information for the abundance model for the Eastern Bluebird (Sialia sialis). The model was a quasi-Poisson GAMM with log link function. Based on QIC, support for including the noise level as a predictor of abundance was: none; support for including the standard deviation of noise was: strong.

\begin{tabular}{lrrr}
\hline name & QIC & delta QIC & Akaike weight \\
\hline No Noise Variability Term & -1863.59 & 0.00 & 1.00 \\
No Mean Noise Term & -961.44 & 902.15 & 0.00 \\
Full Model & 25.18 & 1888.77 & 0.00 \\
No Noise Terms & 299.09 & 2162.67 & 0.00 \\
\hline
\end{tabular}

Table A4.22: Model selection information for the abundance model for the Eastern Meadowlark (Sturnella magna). The model was a quasi-Poisson GAMM with log link function. Based on QIC, support for including the noise level as a predictor of abundance was: strong; support for including the standard deviation of noise was: strong.

\begin{tabular}{lrrr}
\hline name & QIC & delta QIC & Akaike weight \\
\hline No Mean Noise Term & 922.03 & 0.00 & 0.37 \\
Full Model & 922.14 & 0.11 & 0.35 \\
No Noise Variability Term & 923.84 & 1.81 & 0.15 \\
No Noise Terms & 924.01 & 1.98 & 0.14 \\
\hline
\end{tabular}

Table A4.23: Model selection information for the abundance model for the Evening Grosbeak (Coccothraustes vespertinus). The model was a quasi-Poisson GAMM with log link function. Based on QIC, support for including the noise level as a predictor of abundance was: none; support for including the standard deviation of noise was: weak. 


\begin{tabular}{lrrr}
\hline name & QIC & delta QIC & Akaike weight \\
\hline No Noise Variability Term & 4151.57 & 0.00 & 0.46 \\
Full Model & 4151.66 & 0.09 & 0.44 \\
No Noise Terms & 4155.76 & 4.19 & 0.06 \\
No Mean Noise Term & 4155.90 & 4.33 & 0.05 \\
\hline
\end{tabular}

Table A4.24: Model selection information for the abundance model for the Great Crested Flycatcher (Myiarchus crinitus). The model was a quasi-Poisson GAMM with log link function. Based on QIC, support for including the noise level as a predictor of abundance was: strong; support for including the standard deviation of noise was: none.

\begin{tabular}{lrrr}
\hline name & QIC & delta QIC & Akaike weight \\
\hline No Mean Noise Term & 3166.93 & 0.00 & 0.53 \\
Full Model & 3167.28 & 0.35 & 0.44 \\
No Noise Terms & 3173.79 & 6.86 & 0.02 \\
No Noise Variability Term & 3174.83 & 7.90 & 0.01 \\
\hline
\end{tabular}

Table A4.25: Model selection information for the abundance model for the Golden-crowned Kinglet (Regulus satrapa). The model was a quasi-Poisson GAMM with log link function. Based on QIC, support for including the noise level as a predictor of abundance was: none; support for including the standard deviation of noise was: strong.

\begin{tabular}{lrrr}
\hline name & QIC & delta QIC & Akaike weight \\
\hline No Mean Noise Term & 6420.42 & 0.00 & 0.60 \\
No Noise Variability Term & 6421.45 & 1.03 & 0.36 \\
Full Model & 6426.48 & 6.06 & 0.03 \\
No Noise Terms & 6427.34 & 6.92 & 0.02 \\
\hline
\end{tabular}

Table A4.26: Model selection information for the abundance model for the Gray Catbird (Dumetella carolinensis). The model was a quasi-Poisson GAMM with log link function. Based on QIC, support for including the noise level as a predictor of abundance was: none; support for including the standard deviation of noise was: weak.

\begin{tabular}{lrrr}
\hline name & QIC & delta QIC & Akaike weight \\
\hline Full Model & 643.51 & 0.00 & 0.48 \\
No Noise Terms & 644.87 & 1.35 & 0.25 \\
No Mean Noise Term & 645.56 & 2.05 & 0.17 \\
No Noise Variability Term & 646.75 & 3.23 & 0.10 \\
\hline
\end{tabular}

Table A4.27: Model selection information for the abundance model for the Gray Flycatcher (Empidonax wrightii). The model was a quasi-Poisson GAMM with log link function. Based on QIC, support for including the noise level as a predictor of abundance was: weak; support for including the standard deviation of noise was: weak. 


\begin{tabular}{lrrr}
\hline name & QIC & delta QIC & Akaike weight \\
\hline No Mean Noise Term & 3281.26 & 0.00 & 0.39 \\
Full Model & 3281.97 & 0.70 & 0.28 \\
No Noise Terms & 3282.87 & 1.61 & 0.18 \\
No Noise Variability Term & 3283.11 & 1.84 & 0.16 \\
\hline
\end{tabular}

Table A4.28: Model selection information for the abundance model for the Hammond's Flycatcher (Empidonax hammondii). The model was a quasi-Poisson GAMM with log link function. Based on QIC, support for including the noise level as a predictor of abundance was: none; support for including the standard deviation of noise was: weak.

\begin{tabular}{lrrr}
\hline name & QIC & delta QIC & Akaike weight \\
\hline Full Model & 4715.19 & 0.00 & 0.49 \\
No Noise Variability Term & 4715.58 & 0.38 & 0.40 \\
No Noise Terms & 4719.31 & 4.12 & 0.06 \\
No Mean Noise Term & 4719.74 & 4.55 & 0.05 \\
\hline
\end{tabular}

Table A4.29: Model selection information for the abundance model for the Hermit Thrush (Catharus guttatus). The model was a quasi-Poisson GAMM with log link function. Based on QIC, support for including the noise level as a predictor of abundance was: strong; support for including the standard deviation of noise was: weak.

\begin{tabular}{lrrr}
\hline name & QIC & delta QIC & Akaike weight \\
\hline No Noise Variability Term & 3072.36 & 0.00 & 0.29 \\
No Noise Terms & 3072.50 & 0.14 & 0.27 \\
Full Model & 3072.87 & 0.51 & 0.23 \\
No Mean Noise Term & 3073.01 & 0.64 & 0.21 \\
\hline
\end{tabular}

Table A4.30: Model selection information for the abundance model for the House Finch (Haemorhous mexicanus). The model was a quasi-Poisson GAMM with log link function. Based on QIC, support for including the noise level as a predictor of abundance was: weak; support for including the standard deviation of noise was: none. 


\begin{tabular}{lrrr}
\hline name & QIC & delta QIC & Akaike weight \\
\hline No Noise Terms & 843.31 & 0.00 & 0.32 \\
No Mean Noise Term & 843.92 & 0.61 & 0.24 \\
Full Model & 844.00 & 0.69 & 0.23 \\
No Noise Variability Term & 844.05 & 0.74 & 0.22 \\
\hline
\end{tabular}

Table A4.31: Model selection information for the abundance model for the House Sparrow (Passer domesticus). The model was a quasi-Poisson GAMM with log link function. Based on QIC, support for including the noise level as a predictor of abundance was: none; support for including the standard deviation of noise was: none.

\begin{tabular}{lrrr}
\hline name & QIC & delta QIC & Akaike weight \\
\hline No Noise Terms & 5665.85 & 0.00 & 0.31 \\
No Mean Noise Term & 5665.96 & 0.11 & 0.29 \\
No Noise Variability Term & 5666.68 & 0.83 & 0.20 \\
Full Model & 5666.75 & 0.90 & 0.20 \\
\hline
\end{tabular}

Table A4.32: Model selection information for the abundance model for the House Wren (Troglodytes aedon). The model was a quasi-Poisson GAMM with log link function. Based on QIC, support for including the noise level as a predictor of abundance was: none; support for including the standard deviation of noise was: none.

\begin{tabular}{lrrr}
\hline name & QIC & delta QIC & Akaike weight \\
\hline No Mean Noise Term & 5847.38 & 0.00 & 0.57 \\
No Noise Terms & 5847.94 & 0.56 & 0.43 \\
\hline
\end{tabular}

Table A4.33: Model selection information for the abundance model for the Indigo Bunting (Passerina cyanea). The model was a quasi-Poisson GAMM with log link function. Based on QIC, support for including the noise level as a predictor of abundance was: none; support for including the standard deviation of noise was: weak. 


\begin{tabular}{lrrr}
\hline name & QIC & delta QIC & Akaike weight \\
\hline No Noise Terms & 3381.88 & 0.00 & 0.48 \\
No Mean Noise Term & 3382.00 & 0.12 & 0.46 \\
No Noise Variability Term & 3387.31 & 5.43 & 0.03 \\
Full Model & 3387.55 & 5.67 & 0.03 \\
\hline
\end{tabular}

Table A4.34: Model selection information for the abundance model for the Lazuli Bunting (Passerina amoena). The model was a quasi-Poisson GAMM with log link function. Based on QIC, support for including the noise level as a predictor of abundance was: none; support for including the standard deviation of noise was: none.

\begin{tabular}{lrrr}
\hline name & QIC & delta QIC & Akaike weight \\
\hline No Noise Variability Term & 1575.87 & 0.00 & 0.37 \\
No Noise Terms & 1576.08 & 0.22 & 0.33 \\
Full Model & 1577.66 & 1.79 & 0.15 \\
No Mean Noise Term & 1577.72 & 1.86 & 0.15 \\
\hline
\end{tabular}

Table A4.35: Model selection information for the abundance model for the Least Flycatcher (Empidonax minimus). The model was a quasi-Poisson GAMM with log link function. Based on QIC, support for including the noise level as a predictor of abundance was: weak; support for including the standard deviation of noise was: none.

\begin{tabular}{lrrr}
\hline name & QIC & delta QIC & Akaike weight \\
\hline No Noise Variability Term & 2664.05 & 0.00 & 0.30 \\
No Noise Terms & 2664.12 & 0.07 & 0.29 \\
Full Model & 2664.74 & 0.69 & 0.21 \\
No Mean Noise Term & 2664.79 & 0.73 & 0.21 \\
\hline
\end{tabular}

Table A4.36: Model selection information for the abundance model for the Lincoln's Sparrow (Melospiza lincolnii). The model was a quasi-Poisson GAMM with log link function. Based on QIC, support for including the noise level as a predictor of abundance was: weak; support for including the standard deviation of noise was: none.

\begin{tabular}{lrrr}
\hline name & QIC & delta QIC & Akaike weight \\
\hline No Noise Variability Term & 884.08 & 0.00 & 0.40 \\
Full Model & 884.12 & 0.04 & 0.39 \\
No Mean Noise Term & 885.92 & 1.84 & 0.16 \\
No Noise Terms & 887.88 & 3.80 & 0.06 \\
\hline
\end{tabular}

Table A4.37: Model selection information for the abundance model for the Magnolia Warbler (Setophaga magnolia). The model was a quasi-Poisson GAMM with log link function. Based on QIC, support for including the noise level as a predictor of abundance was: weak; support for including the standard deviation of noise was: none. 


\begin{tabular}{lrrr}
\hline name & QIC & delta QIC & Akaike weight \\
\hline No Mean Noise Term & 5614.97 & 0.00 & 0.27 \\
No Noise Terms & 5615.11 & 0.14 & 0.26 \\
Full Model & 5615.18 & 0.21 & 0.25 \\
No Noise Variability Term & 5615.37 & 0.40 & 0.22 \\
\hline
\end{tabular}

Table A4.38: Model selection information for the abundance model for the MacGillivray's Warbler (Geothlypis tolmiei). The model was a quasi-Poisson GAMM with log link function. Based on QIC, support for including the noise level as a predictor of abundance was: none; support for including the standard deviation of noise was: weak.

\begin{tabular}{lrrr}
\hline name & QIC & delta QIC & Akaike weight \\
\hline No Mean Noise Term & 3035.09 & 0.00 & 0.34 \\
No Noise Terms & 3035.30 & 0.21 & 0.30 \\
Full Model & 3036.10 & 1.01 & 0.20 \\
No Noise Variability Term & 3036.58 & 1.48 & 0.16 \\
\hline
\end{tabular}

Table A4.39: Model selection information for the abundance model for the Mountain Chickadee (Poecile gambeli). The model was a quasi-Poisson GAMM with log link function. Based on QIC, support for including the noise level as a predictor of abundance was: none; support for including the standard deviation of noise was: weak.

\begin{tabular}{lrrr}
\hline name & QIC & delta QIC & Akaike weight \\
\hline No Noise Terms & 2079.11 & 0.00 & 0.33 \\
No Noise Variability Term & 2079.74 & 0.64 & 0.24 \\
No Mean Noise Term & 2079.80 & 0.69 & 0.23 \\
Full Model & 2080.17 & 1.06 & 0.19 \\
\hline
\end{tabular}

Table A4.40: Model selection information for the abundance model for the Mourning Dove (Zenaida macroura). The model was a quasi-Poisson GAMM with log link function. Based on QIC, support for including the noise level as a predictor of abundance was: none; support for including the standard deviation of noise was: none. 


\begin{tabular}{lrrr}
\hline name & QIC & delta QIC & Akaike weight \\
\hline No Noise Variability Term & 695.49 & 0.00 & 0.86 \\
Full Model & 699.19 & 3.71 & 0.14 \\
No Mean Noise Term & 726.06 & 30.58 & 0.00 \\
No Noise Terms & 729.09 & 33.61 & 0.00 \\
\hline
\end{tabular}

Table A4.41: Model selection information for the abundance model for the Myrtle Warbler (Setophaga coronata coronata). The model was a quasi-Poisson GAMM with log link function. Based on QIC, support for including the noise level as a predictor of abundance was: strong; support for including the standard deviation of noise was: none.

\begin{tabular}{lrrr}
\hline name & QIC & delta QIC & Akaike weight \\
\hline No Noise Variability Term & 2418.43 & 0.00 & 0.29 \\
No Noise Terms & 2418.51 & 0.08 & 0.27 \\
Full Model & 2418.86 & 0.43 & 0.23 \\
No Mean Noise Term & 2419.05 & 0.63 & 0.21 \\
\hline
\end{tabular}

Table A4.42: Model selection information for the abundance model for the Nashville Warbler (Oreothlypis ruficapilla). The model was a quasi-Poisson GAMM with log link function. Based on QIC, support for including the noise level as a predictor of abundance was: weak; support for including the standard deviation of noise was: none.

\begin{tabular}{lrrr}
\hline name & QIC & delta QIC & Akaike weight \\
\hline No Mean Noise Term & 9974.57 & 0.00 & 0.57 \\
No Noise Terms & 9975.28 & 0.72 & 0.40 \\
Full Model & 9981.77 & 7.21 & 0.02 \\
No Noise Variability Term & 9982.56 & 8.00 & 0.01 \\
\hline
\end{tabular}

Table A4.43: Model selection information for the abundance model for the Northern Cardinal (Cardinalis cardinalis). The model was a quasi-Poisson GAMM with log link function. Based on QIC, support for including the noise level as a predictor of abundance was: none; support for including the standard deviation of noise was: weak. 


\begin{tabular}{lrrr}
\hline name & QIC & delta QIC & Akaike weight \\
\hline Full Model & 3324.69 & 0.00 & 0.42 \\
No Noise Variability Term & 3325.62 & 0.93 & 0.27 \\
No Noise Terms & 3326.63 & 1.94 & 0.16 \\
No Mean Noise Term & 3326.77 & 2.08 & 0.15 \\
\hline
\end{tabular}

Table A4.44: Model selection information for the abundance model for the Orange-crowned Warbler (Oreothlypis celata). The model was a quasi-Poisson GAMM with log link function. Based on QIC, support for including the noise level as a predictor of abundance was: weak; support for including the standard deviation of noise was: weak.

\begin{tabular}{lrrr}
\hline name & QIC & delta QIC & Akaike weight \\
\hline Full Model & 6715.55 & 0.00 & 0.61 \\
No Noise Variability Term & 6716.71 & 1.16 & 0.34 \\
No Mean Noise Term & 6721.23 & 5.68 & 0.04 \\
No Noise Terms & 6722.46 & 6.91 & 0.02 \\
\hline
\end{tabular}

Table A4.45: Model selection information for the abundance model for the Ovenbird (Seiurus aurocapilla). The model was a quasi-Poisson GAMM with log link function. Based on QIC, support for including the noise level as a predictor of abundance was: strong; support for including the standard deviation of noise was: weak.

\begin{tabular}{lrrr}
\hline name & QIC & delta QIC & Akaike weight \\
\hline No Noise Terms & 2463.75 & 0.00 & 0.29 \\
No Noise Variability Term & 2464.06 & 0.30 & 0.25 \\
Full Model & 2464.20 & 0.45 & 0.23 \\
No Mean Noise Term & 2464.29 & 0.54 & 0.22 \\
\hline
\end{tabular}

Table A4.46: Model selection information for the abundance model for the Pine Siskin (Spinus pinus). The model was a quasi-Poisson GAMM with log link function. Based on QIC, support for including the noise level as a predictor of abundance was: none; support for including the standard deviation of noise was: none.

\begin{tabular}{lrrr}
\hline name & QIC & delta QIC & Akaike weight \\
\hline Full Model & 3752.59 & 0.00 & 1.00 \\
No Noise Variability Term & 3771.26 & 18.68 & 0.00 \\
No Mean Noise Term & 3772.56 & 19.98 & 0.00 \\
No Noise Terms & 3778.45 & 25.87 & 0.00 \\
\hline
\end{tabular}

Table A4.47: Model selection information for the abundance model for the Red-breasted Nuthatch (Sitta canadensis). The model was a quasi-Poisson GAMM with log link function. Based on QIC, support for including the noise level as a predictor of abundance was: strong; support for including the standard deviation of noise was: strong. 


\begin{tabular}{lrrr}
\hline name & QIC & delta QIC & Akaike weight \\
\hline No Noise Variability Term & 1586.90 & 0.00 & 0.51 \\
No Noise Terms & 1588.34 & 1.43 & 0.25 \\
Full Model & 1589.20 & 2.30 & 0.16 \\
No Mean Noise Term & 1590.85 & 3.94 & 0.07 \\
\hline
\end{tabular}

Table A4.48: Model selection information for the abundance model for the Ruby-crowned Kinglet (Regulus calendula). The model was a quasi-Poisson GAMM with log link function. Based on QIC, support for including the noise level as a predictor of abundance was: weak; support for including the standard deviation of noise was: none.

\begin{tabular}{lrrr}
\hline name & QIC & delta QIC & Akaike weight \\
\hline Full Model & 7920.33 & 0.00 & 0.48 \\
No Mean Noise Term & 7920.40 & 0.07 & 0.46 \\
No Noise Terms & 7926.03 & 5.70 & 0.03 \\
No Noise Variability Term & 7926.10 & 5.77 & 0.03 \\
\hline
\end{tabular}

Table A4.49: Model selection information for the abundance model for the Red-eyed Vireo (Vireo olivaceus). The model was a quasi-Poisson GAMM with log link function. Based on QIC, support for including the noise level as a predictor of abundance was: weak; support for including the standard deviation of noise was: strong. 


\begin{tabular}{lrrr}
\hline name & QIC & delta QIC & Akaike weight \\
\hline No Noise Terms & 2390.60 & 0.00 & 0.28 \\
No Mean Noise Term & 2390.68 & 0.07 & 0.26 \\
No Noise Variability Term & 2390.89 & 0.28 & 0.24 \\
Full Model & 2391.04 & 0.43 & 0.22 \\
\hline
\end{tabular}

Table A4.50: Model selection information for the abundance model for the Rufous Hummingbird (Selasphorus rufus). The model was a quasi-Poisson GAMM with log link function. Based on QIC, support for including the noise level as a predictor of abundance was: none; support for including the standard deviation of noise was: none.

\begin{tabular}{lrrr}
\hline name & QIC & delta QIC & Akaike weight \\
\hline Full Model & 3710.43 & 0.00 & 0.81 \\
No Noise Variability Term & 3714.78 & 4.35 & 0.09 \\
No Mean Noise Term & 3715.08 & 4.65 & 0.08 \\
No Noise Terms & 3718.66 & 8.23 & 0.01 \\
\hline
\end{tabular}

Table A4.51: Model selection information for the abundance model for the Scarlet Tanager (Piranga olivacea). The model was a quasi-Poisson GAMM with log link function. Based on QIC, support for including the noise level as a predictor of abundance was: strong; support for including the standard deviation of noise was: strong.

\begin{tabular}{lrrr}
\hline name & QIC & delta QIC & Akaike weight \\
\hline No Mean Noise Term & 9455.69 & 0.00 & 0.36 \\
Full Model & 9455.73 & 0.04 & 0.35 \\
No Noise Terms & 9457.49 & 1.80 & 0.15 \\
No Noise Variability Term & 9457.61 & 1.92 & 0.14 \\
\hline
\end{tabular}

Table A4.52: Model selection information for the abundance model for the Song Sparrow (Melospiza melodia). The model was a quasi-Poisson GAMM with log link function. Based on QIC, support for including the noise level as a predictor of abundance was: none; support for including the standard deviation of noise was: weak. 


\begin{tabular}{lrrr}
\hline name & QIC & delta QIC & Akaike weight \\
\hline No Noise Terms & 5325.28 & 0.00 & 0.34 \\
No Mean Noise Term & 5325.63 & 0.35 & 0.28 \\
No Noise Variability Term & 5326.22 & 0.94 & 0.21 \\
Full Model & 5326.63 & 1.35 & 0.17 \\
\hline
\end{tabular}

Table A4.53: Model selection information for the abundance model for the Spotted Towhee (Pipilo maculatus). The model was a quasi-Poisson GAMM with log link function. Based on QIC, support for including the noise level as a predictor of abundance was: none; support for including the standard deviation of noise was: none.

\begin{tabular}{lrrr}
\hline name & QIC & delta QIC & Akaike weight \\
\hline No Noise Terms & 5524.35 & 0.00 & 0.33 \\
No Mean Noise Term & 5524.47 & 0.13 & 0.31 \\
No Noise Variability Term & 5525.50 & 1.16 & 0.19 \\
Full Model & 5525.63 & 1.29 & 0.17 \\
\hline
\end{tabular}

Table A4.54: Model selection information for the abundance model for the Swainson's Thrush (Catharus ustulatus). The model was a quasi-Poisson GAMM with log link function. Based on QIC, support for including the noise level as a predictor of abundance was: none; support for including the standard deviation of noise was: none.

\begin{tabular}{lrrr}
\hline name & QIC & delta QIC & Akaike weight \\
\hline No Mean Noise Term & 865.08 & 0.00 & 0.97 \\
Full Model & 871.95 & 6.86 & 0.03 \\
No Noise Terms & 877.24 & 12.15 & 0.00 \\
No Noise Variability Term & 879.19 & 14.10 & 0.00 \\
\hline
\end{tabular}

Table A4.55: Model selection information for the abundance model for the Townsend's Solitaire (Myadestes townsendi). The model was a quasi-Poisson GAMM with log link function. Based on QIC, support for including the noise level as a predictor of abundance was: none; support for including the standard deviation of noise was: strong.

\begin{tabular}{lrrr}
\hline name & QIC & delta QIC & Akaike weight \\
\hline No Mean Noise Term & 1305.16 & 0.00 & 0.51 \\
No Noise Terms & 1305.25 & 0.08 & 0.48 \\
Full Model & 1313.06 & 7.89 & 0.01 \\
No Noise Variability Term & 1322.69 & 17.53 & 0.00 \\
\hline
\end{tabular}

Table A4.56: Model selection information for the abundance model for the Townsend's Warbler (Setophaga townsendi). The model was a quasi-Poisson GAMM with log link function. Based on QIC, support for including the noise level as a predictor of abundance was: none; support for including the standard deviation of noise was: weak. 


\begin{tabular}{lrrr}
\hline name & QIC & delta QIC & Akaike weight \\
\hline Full Model & 6577.93 & 0.00 & 0.40 \\
No Mean Noise Term & 6578.39 & 0.46 & 0.31 \\
No Noise Terms & 6579.84 & 1.91 & 0.15 \\
No Noise Variability Term & 6580.02 & 2.09 & 0.14 \\
\hline
\end{tabular}

Table A4.57: Model selection information for the abundance model for the Warbling Vireo (Vireo gilvus). The model was a quasi-Poisson GAMM with log link function. Based on QIC, support for including the noise level as a predictor of abundance was: weak; support for including the standard deviation of noise was: weak.

\begin{tabular}{lrrr}
\hline name & QIC & delta QIC & Akaike weight \\
\hline No Noise Variability Term & 4344.52 & 0.00 & 0.58 \\
No Noise Terms & 4346.59 & 2.06 & 0.21 \\
Full Model & 4347.12 & 2.60 & 0.16 \\
No Mean Noise Term & 4349.15 & 4.63 & 0.06 \\
\hline
\end{tabular}

Table A4.58: Model selection information for the abundance model for the White-breasted Nuthatch (Sitta carolinensis). The model was a quasi-Poisson GAMM with log link function. Based on QIC, support for including the noise level as a predictor of abundance was: strong; support for including the standard deviation of noise was: none.

\begin{tabular}{|c|c|c|c|}
\hline name & QIC & delta QIC & Akaike weight \\
\hline No Mean Noise Term & 263.59 & 0.00 & 0.31 \\
\hline Full Model & 263.90 & 0.31 & 0.27 \\
\hline No Noise Terms & 264.20 & 0.61 & 0.23 \\
\hline No Noise Variability Term & 264.55 & 0.96 & 0.19 \\
\hline
\end{tabular}

Table A4.59: Model selection information for the abundance model for the White-crowned Sparrow (Zonotrichia leucophrys). The model was a quasi-Poisson GAMM with log link function. Based on QIC, support for including the noise level as a predictor of abundance was: none; support for including the standard deviation of noise was: weak. 


\begin{tabular}{lrrr}
\hline name & QIC & delta QIC & Akaike weight \\
\hline No Mean Noise Term & 679.61 & 0.00 & 0.50 \\
Full Model & 680.13 & 0.52 & 0.39 \\
No Noise Variability Term & 684.05 & 4.44 & 0.05 \\
No Noise Terms & 684.12 & 4.51 & 0.05 \\
\hline
\end{tabular}

Table A4.60: Model selection information for the abundance model for the Western Bluebird (Sialia mexicana). The model was a quasi-Poisson GAMM with log link function. Based on QIC, support for including the noise level as a predictor of abundance was: none; support for including the standard deviation of noise was: strong.

\begin{tabular}{lrrr}
\hline name & QIC & delta QIC & Akaike weight \\
\hline Full Model & 1784.68 & 0.00 & 0.53 \\
No Noise Variability Term & 1785.35 & 0.66 & 0.38 \\
No Mean Noise Term & 1789.35 & 4.67 & 0.05 \\
No Noise Terms & 1789.90 & 5.22 & 0.04 \\
\hline
\end{tabular}

Table A4.61: Model selection information for the abundance model for the Western ScrubJay (Aphelocoma californica). The model was a quasi-Poisson GAMM with log link function. Based on QIC, support for including the noise level as a predictor of abundance was: strong; support for including the standard deviation of noise was: weak.

\begin{tabular}{lrrr}
\hline name & QIC & delta QIC & Akaike weight \\
\hline No Mean Noise Term & 4407.47 & 0.00 & 0.31 \\
Full Model & 4407.80 & 0.33 & 0.26 \\
No Noise Terms & 4408.04 & 0.57 & 0.23 \\
No Noise Variability Term & 4408.39 & 0.92 & 0.20 \\
\hline
\end{tabular}

Table A4.62: Model selection information for the abundance model for the Western Tanager (Piranga ludoviciana). The model was a quasi-Poisson GAMM with log link function. Based on QIC, support for including the noise level as a predictor of abundance was: none; support for including the standard deviation of noise was: weak. 


\begin{tabular}{lrrr}
\hline name & QIC & delta QIC & Akaike weight \\
\hline No Noise Variability Term & 4131.96 & 0.00 & 0.49 \\
Full Model & 4132.05 & 0.09 & 0.47 \\
No Mean Noise Term & 4137.67 & 5.71 & 0.03 \\
No Noise Terms & 4141.01 & 9.05 & 0.01 \\
\hline
\end{tabular}

Table A4.63: Model selection information for the abundance model for the White-eyed Vireo (Vireo griseus). The model was a quasi-Poisson GAMM with log link function. Based on QIC, support for including the noise level as a predictor of abundance was: strong; support for including the standard deviation of noise was: none.

\begin{tabular}{lrrr}
\hline name & QIC & delta QIC & Akaike weight \\
\hline Full Model & 3990.06 & 0.00 & 0.28 \\
No Noise Variability Term & 3990.28 & 0.22 & 0.25 \\
No Mean Noise Term & 3990.33 & 0.28 & 0.24 \\
No Noise Terms & 3990.36 & 0.30 & 0.24 \\
\hline
\end{tabular}

Table A4.64: Model selection information for the abundance model for the Wilson's Warbler (Cardellina pusilla). The model was a quasi-Poisson GAMM with log link function. Based on QIC, support for including the noise level as a predictor of abundance was: weak; support for including the standard deviation of noise was: weak.

\begin{tabular}{lrrr}
\hline name & QIC & delta QIC & Akaike weight \\
\hline No Noise Variability Term & 5554.55 & 0.00 & 0.46 \\
Full Model & 5555.48 & 0.92 & 0.29 \\
No Noise Terms & 5556.96 & 2.41 & 0.14 \\
No Mean Noise Term & 5557.39 & 2.83 & 0.11 \\
\hline
\end{tabular}

Table A4.65: Model selection information for the abundance model for the Wood Thrush (Hylocichla mustelina). The model was a quasi-Poisson GAMM with log link function. Based on QIC, support for including the noise level as a predictor of abundance was: strong; support for including the standard deviation of noise was: none.

\begin{tabular}{lrrr}
\hline name & QIC & delta QIC & Akaike weight \\
\hline No Noise Terms & 1853.90 & 0.00 & 0.30 \\
No Noise Variability Term & 1854.19 & 0.29 & 0.26 \\
No Mean Noise Term & 1854.25 & 0.35 & 0.25 \\
Full Model & 1854.75 & 0.86 & 0.19 \\
\hline
\end{tabular}

Table A4.66: Model selection information for the abundance model for the Wrentit (Chamaea fasciata). The model was a quasi-Poisson GAMM with log link function. Based on QIC, support for including the noise level as a predictor of abundance was: none; support for including the standard deviation of noise was: none. 


\begin{tabular}{lrrr}
\hline name & QIC & delta QIC & Akaike weight \\
\hline No Mean Noise Term & 703.62 & 0.00 & 0.43 \\
Full Model & 703.86 & 0.24 & 0.38 \\
No Noise Variability Term & 705.56 & 1.94 & 0.16 \\
No Noise Terms & 709.59 & 5.97 & 0.02 \\
\hline
\end{tabular}

Table A4.67: Model selection information for the abundance model for the White-throated Sparrow (Zonotrichia albicollis). The model was a quasi-Poisson GAMM with log link function. Based on QIC, support for including the noise level as a predictor of abundance was: none; support for including the standard deviation of noise was: weak.

\begin{tabular}{lrrr}
\hline name & QIC & delta QIC & Akaike weight \\
\hline Full Model & 4792.71 & 0.00 & 0.51 \\
No Mean Noise Term & 4794.37 & 1.66 & 0.22 \\
No Noise Variability Term & 4795.27 & 2.56 & 0.14 \\
No Noise Terms & 4795.64 & 2.93 & 0.12 \\
\hline
\end{tabular}

Table A4.68: Model selection information for the abundance model for the Yellow-breasted Chat (Icteria virens). The model was a quasi-Poisson GAMM with log link function. Based on QIC, support for including the noise level as a predictor of abundance was: weak; support for including the standard deviation of noise was: strong.

\begin{tabular}{lrrr}
\hline name & QIC & delta QIC & Akaike weight \\
\hline No Mean Noise Term & 3157.60 & 0.00 & 0.29 \\
No Noise Terms & 3157.97 & 0.37 & 0.24 \\
Full Model & 3157.99 & 0.39 & 0.24 \\
No Noise Variability Term & 3158.19 & 0.59 & 0.22 \\
\hline
\end{tabular}

Table A4.69: Model selection information for the abundance model for the Yellow-billed Cuckoo (Coccyzus americanus). The model was a quasi-Poisson GAMM with log link function. Based on QIC, support for including the noise level as a predictor of abundance was: none; support for including the standard deviation of noise was: weak. 


\begin{tabular}{lrrr}
\hline name & QIC & delta QIC & Akaike weight \\
\hline No Noise Variability Term & 6259.08 & 0.00 & 0.32 \\
Full Model & 6259.28 & 0.20 & 0.29 \\
No Mean Noise Term & 6260.04 & 0.96 & 0.20 \\
No Noise Terms & 6260.18 & 1.10 & 0.19 \\
\hline
\end{tabular}

Table A4.70: Model selection information for the abundance model for the Yellow Warbler (Setophaga petechia). The model was a quasi-Poisson GAMM with log link function. Based on QIC, support for including the noise level as a predictor of abundance was: weak; support for including the standard deviation of noise was: none.

\section{Productivity Models}

\begin{tabular}{lrrr}
\hline name & QIC & delta QIC & Akaike weight \\
\hline No Mean Noise Term & 1843.32 & 0.00 & 0.47 \\
No Noise Terms & 1844.53 & 1.21 & 0.26 \\
Full Model & 1845.77 & 2.44 & 0.14 \\
No Noise Variability Term & 1845.91 & 2.59 & 0.13 \\
\hline
\end{tabular}

Table A4.71: Model selection information for the productivity model for the Acadian Flycatcher (Empidonax virescens). The model was a quasi-Poisson GAMM with log link function. Based on QIC, support for including the noise level as a predictor of productivity was: none; support for including the standard deviation of noise was: weak.

\begin{tabular}{lrrr}
\hline name & QIC & delta QIC & Akaike weight \\
\hline No Noise Terms & 1536.66 & 0.00 & 0.34 \\
No Mean Noise Term & 1537.36 & 0.69 & 0.24 \\
No Noise Variability Term & 1537.36 & 0.70 & 0.24 \\
Full Model & 1538.06 & 1.40 & 0.17 \\
\hline
\end{tabular}

Table A4.72: Model selection information for the productivity model for the American Goldfinch (Spinus tristis). The model was a quasi-Poisson GAMM with log link function. Based on QIC, support for including the noise level as a predictor of productivity was: none; support for including the standard deviation of noise was: none. 


\begin{tabular}{lrrr}
\hline name & QIC & delta QIC & Akaike weight \\
\hline No Noise Variability Term & 1422.97 & 0.00 & 0.53 \\
Full Model & 1423.55 & 0.57 & 0.40 \\
No Noise Terms & 1428.07 & 5.09 & 0.04 \\
No Mean Noise Term & 1428.77 & 5.80 & 0.03 \\
\hline
\end{tabular}

Table A4.73: Model selection information for the productivity model for the American Redstart (Setophaga ruticilla). The model was a quasi-Poisson GAMM with log link function. Based on QIC, support for including the noise level as a predictor of productivity was: strong; support for including the standard deviation of noise was: none.

\begin{tabular}{lrrr}
\hline name & QIC & delta QIC & Akaike weight \\
\hline No Noise Variability Term & 5457.13 & 0.00 & 0.61 \\
Full Model & 5459.03 & 1.90 & 0.24 \\
No Noise Terms & 5460.80 & 3.68 & 0.10 \\
No Mean Noise Term & 5461.89 & 4.76 & 0.06 \\
\hline
\end{tabular}

Table A4.74: Model selection information for the productivity model for the American Robin (Turdus migratorius). The model was a quasi-Poisson GAMM with log link function. Based on QIC, support for including the noise level as a predictor of productivity was: strong; support for including the standard deviation of noise was: none.

\begin{tabular}{lrrr}
\hline name & QIC & delta QIC & Akaike weight \\
\hline No Noise Terms & 942.14 & 0.00 & 0.43 \\
No Noise Variability Term & 942.58 & 0.43 & 0.35 \\
No Mean Noise Term & 944.55 & 2.41 & 0.13 \\
Full Model & 945.21 & 3.07 & 0.09 \\
\hline
\end{tabular}

Table A4.75: Model selection information for the productivity model for the Ash-throated Flycatcher (Myiarchus cinerascens). The model was a quasi-Poisson GAMM with log link function. Based on QIC, support for including the noise level as a predictor of productivity was: none; support for including the standard deviation of noise was: none.

\begin{tabular}{lrrr}
\hline name & QIC & delta QIC & Akaike weight \\
\hline No Noise Terms & 1400.59 & 0.00 & 0.31 \\
No Mean Noise Term & 1400.98 & 0.39 & 0.26 \\
No Noise Variability Term & 1401.14 & 0.54 & 0.24 \\
Full Model & 1401.52 & 0.93 & 0.20 \\
\hline
\end{tabular}

Table A4.76: Model selection information for the productivity model for the Audubon's Warbler (Setophaga coronata auduboni). The model was a quasi-Poisson GAMM with log link function. Based on QIC, support for including the noise level as a predictor of productivity was: none; support for including the standard deviation of noise was: none. 


\begin{tabular}{lrrr}
\hline name & QIC & delta QIC & Akaike weight \\
\hline No Mean Noise Term & 2762.70 & 0.00 & 0.37 \\
Full Model & 2762.88 & 0.18 & 0.34 \\
No Noise Terms & 2764.42 & 1.72 & 0.16 \\
No Noise Variability Term & 2764.77 & 2.07 & 0.13 \\
\hline
\end{tabular}

Table A4.77: Model selection information for the productivity model for the Black-capped Chickadee (Poecile atricapillus). The model was a quasi-Poisson GAMM with log link function. Based on QIC, support for including the noise level as a predictor of productivity was: none; support for including the standard deviation of noise was: strong.

\begin{tabular}{lrrr}
\hline name & QIC & delta QIC & Akaike weight \\
\hline No Noise Terms & 650.00 & 0.00 & 0.38 \\
No Mean Noise Term & 650.58 & 0.59 & 0.29 \\
No Noise Variability Term & 651.19 & 1.19 & 0.21 \\
Full Model & 652.32 & 2.33 & 0.12 \\
\hline
\end{tabular}

Table A4.78: Model selection information for the productivity model for the Black-chinned Hummingbird (Archilochus alexandri). The model was a quasi-Poisson GAMM with log link function. Based on QIC, support for including the noise level as a predictor of productivity was: none; support for including the standard deviation of noise was: none.

\begin{tabular}{lrrr}
\hline name & QIC & delta QIC & Akaike weight \\
\hline No Noise Terms & 1752.30 & 0.00 & 0.34 \\
No Noise Variability Term & 1752.93 & 0.63 & 0.25 \\
No Mean Noise Term & 1753.00 & 0.70 & 0.24 \\
Full Model & 1753.74 & 1.44 & 0.17 \\
\hline
\end{tabular}

Table A4.79: Model selection information for the productivity model for the Bewick's Wren (Thryomanes bewickii). The model was a quasi-Poisson GAMM with log link function. Based on QIC, support for including the noise level as a predictor of productivity was: none; support for including the standard deviation of noise was: none.

\begin{tabular}{lrrr}
\hline name & QIC & delta QIC & Akaike weight \\
\hline Full Model & 2527.20 & 0.00 & 0.33 \\
No Noise Variability Term & 2527.37 & 0.17 & 0.30 \\
No Mean Noise Term & 2528.00 & 0.80 & 0.22 \\
No Noise Terms & 2528.87 & 1.67 & 0.14 \\
\hline
\end{tabular}

Table A4.80: Model selection information for the productivity model for the Black-headed Grosbeak (Pheucticus melanocephalus). The model was a quasi-Poisson GAMM with log link function. Based on QIC, support for including the noise level as a predictor of productivity was: weak; support for including the standard deviation of noise was: weak. 


\begin{tabular}{lrrr}
\hline name & QIC & delta QIC & Akaike weight \\
\hline No Noise Terms & 129.96 & 0.00 & 0.36 \\
No Mean Noise Term & 130.44 & 0.48 & 0.28 \\
Full Model & 131.15 & 1.19 & 0.20 \\
No Noise Variability Term & 131.44 & 1.49 & 0.17 \\
\hline
\end{tabular}

Table A4.81: Model selection information for the productivity model for the Bobolink (Dolichonyx oryzivorus). The model was a quasi-Poisson GAMM with log link function. Based on QIC, support for including the noise level as a predictor of productivity was: none; support for including the standard deviation of noise was: none.

\begin{tabular}{lrrr}
\hline name & QIC & delta QIC & Akaike weight \\
\hline No Noise Terms & 1090.93 & 0.00 & 0.37 \\
No Noise Variability Term & 1091.64 & 0.71 & 0.26 \\
No Mean Noise Term & 1091.92 & 0.99 & 0.22 \\
Full Model & 1092.66 & 1.73 & 0.15 \\
\hline
\end{tabular}

Table A4.82: Model selection information for the productivity model for the Bushtit (Psaltriparus minimus). The model was a quasi-Poisson GAMM with log link function. Based on QIC, support for including the noise level as a predictor of productivity was: none; support for including the standard deviation of noise was: none.

\begin{tabular}{lrrr}
\hline name & QIC & delta QIC & Akaike weight \\
\hline No Mean Noise Term & 487.58 & 0.00 & 0.32 \\
Full Model & 488.19 & 0.61 & 0.23 \\
No Noise Terms & 488.24 & 0.66 & 0.23 \\
No Noise Variability Term & 488.34 & 0.76 & 0.22 \\
\hline
\end{tabular}

Table A4.83: Model selection information for the productivity model for the Cassin's Finch (Haemorhous cassinii). The model was a quasi-Poisson GAMM with log link function. Based on QIC, support for including the noise level as a predictor of productivity was: none; support for including the standard deviation of noise was: weak. 


\begin{tabular}{lrrr}
\hline name & QIC & delta QIC & Akaike weight \\
\hline Full Model & 2880.80 & 0.00 & 0.27 \\
No Mean Noise Term & 2880.80 & 0.01 & 0.27 \\
No Noise Terms & 2881.17 & 0.37 & 0.23 \\
No Noise Variability Term & 2881.19 & 0.40 & 0.22 \\
\hline
\end{tabular}

Table A4.84: Model selection information for the productivity model for the Carolina Wren (Thryothorus ludovicianus). The model was a quasi-Poisson GAMM with log link function. Based on QIC, support for including the noise level as a predictor of productivity was: weak; support for including the standard deviation of noise was: weak.

\begin{tabular}{lrrr}
\hline name & QIC & delta QIC & Akaike weight \\
\hline Full Model & 916.74 & 0.00 & 0.97 \\
No Noise Variability Term & 924.10 & 7.36 & 0.02 \\
No Noise Terms & 926.61 & 9.86 & 0.01 \\
No Mean Noise Term & 933.22 & 16.48 & 0.00 \\
\hline
\end{tabular}

Table A4.85: Model selection information for the productivity model for the Cassin's Vireo (Vireo cassinii). The model was a quasi-Poisson GAMM with log link function. Based on QIC, support for including the noise level as a predictor of productivity was: strong; support for including the standard deviation of noise was: strong.

\begin{tabular}{lrrr}
\hline name & QIC & delta QIC & Akaike weight \\
\hline Full Model & 1136.37 & 0.00 & 0.91 \\
No Mean Noise Term & 1141.74 & 5.37 & 0.06 \\
No Noise Terms & 1143.86 & 7.49 & 0.02 \\
No Noise Variability Term & 1148.07 & 11.70 & 0.00 \\
\hline
\end{tabular}

Table A4.86: Model selection information for the productivity model for the Cedar Waxwing (Bombycilla cedrorum). The model was a quasi-Poisson GAMM with log link function. Based on QIC, support for including the noise level as a predictor of productivity was: strong; support for including the standard deviation of noise was: strong.

\begin{tabular}{lrrr}
\hline name & QIC & delta QIC & Akaike weight \\
\hline No Mean Noise Term & 1623.25 & 0.00 & 0.34 \\
No Noise Terms & 1623.46 & 0.21 & 0.30 \\
Full Model & 1624.39 & 1.14 & 0.19 \\
No Noise Variability Term & 1624.59 & 1.34 & 0.17 \\
\hline
\end{tabular}

Table A4.87: Model selection information for the productivity model for the Chipping Sparrow (Spizella passerina). The model was a quasi-Poisson GAMM with log link function. Based on QIC, support for including the noise level as a predictor of productivity was: none; support for including the standard deviation of noise was: weak. 


\begin{tabular}{lrrr}
\hline name & QIC & delta QIC & Akaike weight \\
\hline No Noise Terms & 4399.42 & 0.00 & 0.42 \\
No Mean Noise Term & 4399.44 & 0.02 & 0.41 \\
Full Model & 4402.53 & 3.12 & 0.09 \\
No Noise Variability Term & 4402.70 & 3.28 & 0.08 \\
\hline
\end{tabular}

Table A4.88: Model selection information for the productivity model for the Common Yellowthroat (Geothlypis trichas). The model was a quasi-Poisson GAMM with log link function. Based on QIC, support for including the noise level as a predictor of productivity was: none; support for including the standard deviation of noise was: none.

\begin{tabular}{lrrr}
\hline name & QIC & delta QIC & Akaike weight \\
\hline No Noise Variability Term & 1968.43 & 0.00 & 0.30 \\
No Noise Terms & 1968.60 & 0.18 & 0.27 \\
Full Model & 1968.91 & 0.49 & 0.23 \\
No Mean Noise Term & 1969.24 & 0.81 & 0.20 \\
\hline
\end{tabular}

Table A4.89: Model selection information for the productivity model for the Dark-eyed Junco (Junco hyemalis). The model was a quasi-Poisson GAMM with log link function. Based on QIC, support for including the noise level as a predictor of productivity was: weak; support for including the standard deviation of noise was: none.

\begin{tabular}{lrrr}
\hline name & QIC & delta QIC & Akaike weight \\
\hline No Noise Variability Term & 1154.26 & 0.00 & 0.37 \\
Full Model & 1155.08 & 0.82 & 0.24 \\
No Noise Terms & 1155.10 & 0.85 & 0.24 \\
No Mean Noise Term & 1156.03 & 1.77 & 0.15 \\
\hline
\end{tabular}

Table A4.90: Model selection information for the productivity model for the Dusky Flycatcher (Empidonax oberholseri). The model was a quasi-Poisson GAMM with log link function. Based on QIC, support for including the noise level as a predictor of productivity was: weak; support for including the standard deviation of noise was: none. 


\begin{tabular}{lrrr}
\hline name & QIC & delta QIC & Akaike weight \\
\hline No Mean Noise Term & 592.17 & 0.00 & 0.48 \\
Full Model & 593.05 & 0.88 & 0.31 \\
No Noise Terms & 594.94 & 2.78 & 0.12 \\
No Noise Variability Term & 595.53 & 3.37 & 0.09 \\
\hline
\end{tabular}

Table A4.91: Model selection information for the productivity model for the Eastern Bluebird (Sialia sialis). The model was a quasi-Poisson GAMM with log link function. Based on QIC, support for including the noise level as a predictor of productivity was: none; support for including the standard deviation of noise was: strong.

\begin{tabular}{lrrr}
\hline name & QIC & delta QIC & Akaike weight \\
\hline Full Model & 227.66 & 0.00 & 0.89 \\
No Mean Noise Term & 232.86 & 5.20 & 0.07 \\
No Noise Variability Term & 234.27 & 6.61 & 0.03 \\
No Noise Terms & 236.23 & 8.57 & 0.01 \\
\hline
\end{tabular}

Table A4.92: Model selection information for the productivity model for the Evening Grosbeak (Coccothraustes vespertinus). The model was a quasi-Poisson GAMM with log link function. Based on QIC, support for including the noise level as a predictor of productivity was: strong; support for including the standard deviation of noise was: strong.

\begin{tabular}{lrrr}
\hline name & QIC & delta QIC & Akaike weight \\
\hline No Mean Noise Term & 539.42 & 0.00 & 0.46 \\
Full Model & 540.11 & 0.69 & 0.33 \\
No Noise Terms & 542.03 & 2.61 & 0.13 \\
No Noise Variability Term & 542.80 & 3.37 & 0.09 \\
\hline
\end{tabular}

Table A4.93: Model selection information for the productivity model for the Great Crested Flycatcher (Myiarchus crinitus). The model was a quasi-Poisson GAMM with log link function. Based on QIC, support for including the noise level as a predictor of productivity was: none; support for including the standard deviation of noise was: strong. 


\begin{tabular}{lrrr}
\hline name & QIC & delta QIC & Akaike weight \\
\hline No Noise Terms & 714.71 & 0.00 & 0.31 \\
No Mean Noise Term & 715.00 & 0.29 & 0.27 \\
No Noise Variability Term & 715.38 & 0.67 & 0.22 \\
Full Model & 715.70 & 0.99 & 0.19 \\
\hline
\end{tabular}

Table A4.94: Model selection information for the productivity model for the Golden-crowned Kinglet (Regulus satrapa). The model was a quasi-Poisson GAMM with log link function. Based on QIC, support for including the noise level as a predictor of productivity was: none; support for including the standard deviation of noise was: none.

\begin{tabular}{lrrr}
\hline name & QIC & delta QIC & Akaike weight \\
\hline No Mean Noise Term & 3931.07 & 0.00 & 0.43 \\
Full Model & 3931.37 & 0.29 & 0.37 \\
No Noise Terms & 3933.67 & 2.59 & 0.12 \\
No Noise Variability Term & 3934.20 & 3.12 & 0.09 \\
\hline
\end{tabular}

Table A4.95: Model selection information for the productivity model for the Gray Catbird (Dumetella carolinensis). The model was a quasi-Poisson GAMM with log link function. Based on QIC, support for including the noise level as a predictor of productivity was: none; support for including the standard deviation of noise was: strong.

\begin{tabular}{lrrr}
\hline name & QIC & delta QIC & Akaike weight \\
\hline No Mean Noise Term & 16.17 & 0.00 & 1.00 \\
No Noise Terms & 76.11 & 59.94 & 0.00 \\
No Noise Variability Term & 164.10 & 147.93 & 0.00 \\
Full Model & 167.18 & 151.01 & 0.00 \\
\hline
\end{tabular}

Table A4.96: Model selection information for the productivity model for the Gray Flycatcher (Empidonax wrightii). The model was a quasi-Poisson GAMM with log link function. Based on QIC, support for including the noise level as a predictor of productivity was: strong; support for including the standard deviation of noise was: strong.

\begin{tabular}{lrrr}
\hline name & QIC & delta QIC & Akaike weight \\
\hline Full Model & 1047.18 & 0.00 & 0.30 \\
No Noise Variability Term & 1047.33 & 0.15 & 0.28 \\
No Mean Noise Term & 1047.88 & 0.70 & 0.21 \\
No Noise Terms & 1047.95 & 0.77 & 0.21 \\
\hline
\end{tabular}

Table A4.97: Model selection information for the productivity model for the Hammond's Flycatcher (Empidonax hammondii). The model was a quasi-Poisson GAMM with log link function. Based on QIC, support for including the noise level as a predictor of productivity was: weak; support for including the standard deviation of noise was: weak. 


\begin{tabular}{lrrr}
\hline name & QIC & delta QIC & Akaike weight \\
\hline No Noise Variability Term & 1490.97 & 0.00 & 0.65 \\
Full Model & 1493.03 & 2.06 & 0.23 \\
No Noise Terms & 1494.94 & 3.97 & 0.09 \\
No Mean Noise Term & 1497.45 & 6.48 & 0.03 \\
\hline
\end{tabular}

Table A4.98: Model selection information for the productivity model for the Hermit Thrush (Catharus guttatus). The model was a quasi-Poisson GAMM with log link function. Based on QIC, support for including the noise level as a predictor of productivity was: strong; support for including the standard deviation of noise was: none.

\begin{tabular}{lrrr}
\hline name & QIC & delta QIC & Akaike weight \\
\hline No Mean Noise Term & 1076.49 & 0.00 & 0.29 \\
No Noise Terms & 1076.66 & 0.18 & 0.27 \\
Full Model & 1076.99 & 0.50 & 0.23 \\
No Noise Variability Term & 1077.21 & 0.72 & 0.21 \\
\hline
\end{tabular}

Table A4.99: Model selection information for the productivity model for the House Finch (Haemorhous mexicanus). The model was a quasi-Poisson GAMM with log link function. Based on QIC, support for including the noise level as a predictor of productivity was: none; support for including the standard deviation of noise was: weak. 


\begin{tabular}{lrrr}
\hline name & QIC & delta QIC & Akaike weight \\
\hline No Mean Noise Term & 201.90 & 0.00 & 0.36 \\
Full Model & 202.45 & 0.55 & 0.27 \\
No Noise Variability Term & 202.58 & 0.68 & 0.25 \\
No Noise Terms & 204.08 & 2.18 & 0.12 \\
\hline
\end{tabular}

Table A4.100: Model selection information for the productivity model for the House Sparrow (Passer domesticus). The model was a quasi-Poisson GAMM with log link function. Based on QIC, support for including the noise level as a predictor of productivity was: none; support for including the standard deviation of noise was: weak.

\begin{tabular}{lrrr}
\hline name & QIC & delta QIC & Akaike weight \\
\hline No Noise Variability Term & 2258.03 & 0.00 & 0.37 \\
No Noise Terms & 2258.72 & 0.69 & 0.26 \\
Full Model & 2259.14 & 1.12 & 0.21 \\
No Mean Noise Term & 2259.82 & 1.80 & 0.15 \\
\hline
\end{tabular}

Table A4.101: Model selection information for the productivity model for the House Wren (Troglodytes aedon). The model was a quasi-Poisson GAMM with log link function. Based on QIC, support for including the noise level as a predictor of productivity was: weak; support for including the standard deviation of noise was: none.

\begin{tabular}{lrrr}
\hline name & QIC & delta QIC & Akaike weight \\
\hline No Mean Noise Term & 2544.97 & 0.00 & 0.38 \\
Full Model & 2545.43 & 0.45 & 0.30 \\
No Noise Terms & 2546.51 & 1.53 & 0.18 \\
No Noise Variability Term & 2546.87 & 1.90 & 0.15 \\
\hline
\end{tabular}

Table A4.102: Model selection information for the productivity model for the Indigo Bunting (Passerina cyanea). The model was a quasi-Poisson GAMM with log link function. Based on QIC, support for including the noise level as a predictor of productivity was: none; support for including the standard deviation of noise was: weak. 


\begin{tabular}{lrrr}
\hline name & QIC & delta QIC & Akaike weight \\
\hline No Mean Noise Term & 1589.59 & 0.00 & 0.34 \\
Full Model & 1589.88 & 0.29 & 0.29 \\
No Noise Terms & 1590.68 & 1.09 & 0.20 \\
No Noise Variability Term & 1590.87 & 1.28 & 0.18 \\
\hline
\end{tabular}

Table A4.103: Model selection information for the productivity model for the Lazuli Bunting (Passerina amoena). The model was a quasi-Poisson GAMM with log link function. Based on QIC, support for including the noise level as a predictor of productivity was: none; support for including the standard deviation of noise was: weak.

\begin{tabular}{lrrr}
\hline name & QIC & delta QIC & Akaike weight \\
\hline No Noise Terms & 531.84 & 0.00 & 0.58 \\
No Noise Variability Term & 532.56 & 0.72 & 0.41 \\
Full Model & 541.15 & 9.31 & 0.01 \\
No Mean Noise Term & 541.25 & 9.40 & 0.01 \\
\hline
\end{tabular}

Table A4.104: Model selection information for the productivity model for the Least Flycatcher (Empidonax minimus). The model was a quasi-Poisson GAMM with log link function. Based on QIC, support for including the noise level as a predictor of productivity was: none; support for including the standard deviation of noise was: none.

\begin{tabular}{lrrr}
\hline name & QIC & delta QIC & Akaike weight \\
\hline No Noise Variability Term & 1175.64 & 0.00 & 0.44 \\
Full Model & 1175.96 & 0.33 & 0.37 \\
No Mean Noise Term & 1178.02 & 2.38 & 0.13 \\
No Noise Terms & 1179.53 & 3.89 & 0.06 \\
\hline
\end{tabular}

Table A4.105: Model selection information for the productivity model for the Lincoln's Sparrow (Melospiza lincolnii). The model was a quasi-Poisson GAMM with log link function. Based on QIC, support for including the noise level as a predictor of productivity was: strong; support for including the standard deviation of noise was: none.

\begin{tabular}{lrrr}
\hline name & QIC & delta QIC & Akaike weight \\
\hline No Noise Terms & 365.84 & 0.00 & 0.52 \\
No Mean Noise Term & 366.19 & 0.35 & 0.44 \\
No Noise Variability Term & 371.61 & 5.77 & 0.03 \\
Full Model & 373.75 & 7.91 & 0.01 \\
\hline
\end{tabular}

Table A4.106: Model selection information for the productivity model for the Magnolia Warbler (Setophaga magnolia). The model was a quasi-Poisson GAMM with log link function. Based on QIC, support for including the noise level as a predictor of productivity was: none; support for including the standard deviation of noise was: none. 


\begin{tabular}{lrrr}
\hline name & QIC & delta QIC & Akaike weight \\
\hline No Noise Variability Term & 2963.47 & 0.00 & 0.47 \\
Full Model & 2963.48 & 0.00 & 0.47 \\
No Mean Noise Term & 2968.44 & 4.97 & 0.04 \\
No Noise Terms & 2970.49 & 7.02 & 0.01 \\
\hline
\end{tabular}

Table A4.107: Model selection information for the productivity model for the MacGillivray's Warbler (Geothlypis tolmiei). The model was a quasi-Poisson GAMM with log link function. Based on QIC, support for including the noise level as a predictor of productivity was: strong; support for including the standard deviation of noise was: none.

\begin{tabular}{lrrr}
\hline name & QIC & delta QIC & Akaike weight \\
\hline No Noise Terms & 844.93 & 0.00 & 0.47 \\
No Mean Noise Term & 845.40 & 0.47 & 0.37 \\
Full Model & 848.25 & 3.32 & 0.09 \\
No Noise Variability Term & 848.59 & 3.66 & 0.07 \\
\hline
\end{tabular}

Table A4.108: Model selection information for the productivity model for the Mountain Chickadee (Poecile gambeli). The model was a quasi-Poisson GAMM with log link function. Based on QIC, support for including the noise level as a predictor of productivity was: none; support for including the standard deviation of noise was: none.

\begin{tabular}{lrrr}
\hline name & QIC & delta QIC & Akaike weight \\
\hline No Noise Terms & 369.68 & 0.00 & 0.35 \\
No Mean Noise Term & 370.42 & 0.75 & 0.24 \\
No Noise Variability Term & 370.44 & 0.76 & 0.24 \\
Full Model & 371.19 & 1.51 & 0.17 \\
\hline
\end{tabular}

Table A4.109: Model selection information for the productivity model for the Mourning Dove (Zenaida macroura). The model was a quasi-Poisson GAMM with log link function. Based on QIC, support for including the noise level as a predictor of productivity was: none; support for including the standard deviation of noise was: none. 


\begin{tabular}{lrrr}
\hline name & QIC & delta QIC & Akaike weight \\
\hline Full Model & 163.34 & 0.00 & 1.00 \\
No Noise Variability Term & 177.48 & 14.15 & 0.00 \\
No Mean Noise Term & 193.23 & 29.89 & 0.00 \\
No Noise Terms & 194.86 & 31.52 & 0.00 \\
\hline
\end{tabular}

Table A4.110: Model selection information for the productivity model for the Myrtle Warbler (Setophaga coronata coronata). The model was a quasi-Poisson GAMM with log link function. Based on QIC, support for including the noise level as a predictor of productivity was: strong; support for including the standard deviation of noise was: strong.

\begin{tabular}{lrrr}
\hline name & QIC & delta QIC & Akaike weight \\
\hline Full Model & 844.90 & 0.00 & 0.39 \\
No Noise Variability Term & 844.93 & 0.03 & 0.38 \\
No Mean Noise Term & 847.24 & 2.34 & 0.12 \\
No Noise Terms & 847.50 & 2.61 & 0.11 \\
\hline
\end{tabular}

Table A4.111: Model selection information for the productivity model for the Nashville Warbler (Oreothlypis ruficapilla). The model was a quasi-Poisson GAMM with log link function. Based on QIC, support for including the noise level as a predictor of productivity was: strong; support for including the standard deviation of noise was: weak.

\begin{tabular}{lrrr}
\hline name & QIC & delta QIC & Akaike weight \\
\hline No Noise Variability Term & 4618.19 & 0.00 & 0.50 \\
Full Model & 4618.27 & 0.08 & 0.48 \\
No Noise Terms & 4625.73 & 7.54 & 0.01 \\
No Mean Noise Term & 4625.85 & 7.66 & 0.01 \\
\hline
\end{tabular}

Table A4.112: Model selection information for the productivity model for the Northern Cardinal (Cardinalis cardinalis). The model was a quasi-Poisson GAMM with log link function. Based on QIC, support for including the noise level as a predictor of productivity was: strong; support for including the standard deviation of noise was: none. 


\begin{tabular}{lrrr}
\hline name & QIC & delta QIC & Akaike weight \\
\hline No Mean Noise Term & 1661.56 & 0.00 & 0.30 \\
No Noise Terms & 1661.69 & 0.14 & 0.28 \\
No Noise Variability Term & 1662.26 & 0.70 & 0.21 \\
Full Model & 1662.31 & 0.76 & 0.21 \\
\hline
\end{tabular}

Table A4.113: Model selection information for the productivity model for the Orangecrowned Warbler (Oreothlypis celata). The model was a quasi-Poisson GAMM with log link function. Based on QIC, support for including the noise level as a predictor of productivity was: none; support for including the standard deviation of noise was: weak.

\begin{tabular}{lrrr}
\hline name & QIC & delta QIC & Akaike weight \\
\hline No Mean Noise Term & 3068.95 & 0.00 & 0.52 \\
Full Model & 3069.94 & 0.99 & 0.32 \\
No Noise Terms & 3072.34 & 3.39 & 0.10 \\
No Noise Variability Term & 3073.32 & 4.37 & 0.06 \\
\hline
\end{tabular}

Table A4.114: Model selection information for the productivity model for the Ovenbird (Seiurus aurocapilla). The model was a quasi-Poisson GAMM with log link function. Based on QIC, support for including the noise level as a predictor of productivity was: none; support for including the standard deviation of noise was: strong.

\begin{tabular}{lrrr}
\hline name & QIC & delta QIC & Akaike weight \\
\hline No Noise Variability Term & 730.25 & 0.00 & 0.30 \\
No Noise Terms & 730.43 & 0.18 & 0.28 \\
Full Model & 730.77 & 0.52 & 0.23 \\
No Mean Noise Term & 731.19 & 0.94 & 0.19 \\
\hline
\end{tabular}

Table A4.115: Model selection information for the productivity model for the Pine Siskin (Spinus pinus). The model was a quasi-Poisson GAMM with log link function. Based on QIC, support for including the noise level as a predictor of productivity was: weak; support for including the standard deviation of noise was: none.

\begin{tabular}{lrrr}
\hline name & QIC & delta QIC & Akaike weight \\
\hline No Mean Noise Term & 1144.90 & 0.00 & 0.43 \\
No Noise Terms & 1146.08 & 1.19 & 0.24 \\
Full Model & 1146.25 & 1.35 & 0.22 \\
No Noise Variability Term & 1147.72 & 2.82 & 0.11 \\
\hline
\end{tabular}

Table A4.116: Model selection information for the productivity model for the Red-breasted Nuthatch (Sitta canadensis). The model was a quasi-Poisson GAMM with log link function. Based on QIC, support for including the noise level as a predictor of productivity was: none; support for including the standard deviation of noise was: weak. 


\begin{tabular}{lrrr}
\hline name & QIC & delta QIC & Akaike weight \\
\hline No Noise Terms & 493.59 & 0.00 & 0.32 \\
No Mean Noise Term & 494.09 & 0.50 & 0.25 \\
No Noise Variability Term & 494.35 & 0.76 & 0.22 \\
Full Model & 494.53 & 0.95 & 0.20 \\
\hline
\end{tabular}

Table A4.117: Model selection information for the productivity model for the Ruby-crowned Kinglet (Regulus calendula). The model was a quasi-Poisson GAMM with log link function. Based on QIC, support for including the noise level as a predictor of productivity was: none; support for including the standard deviation of noise was: none.

\begin{tabular}{lrrr}
\hline name & QIC & delta QIC & Akaike weight \\
\hline Full Model & 2493.06 & 0.00 & 0.58 \\
No Mean Noise Term & 2493.79 & 0.73 & 0.40 \\
No Noise Variability Term & 2501.88 & 8.82 & 0.01 \\
No Noise Terms & 2502.65 & 9.59 & 0.00 \\
\hline
\end{tabular}

Table A4.118: Model selection information for the productivity model for the Red-eyed Vireo (Vireo olivaceus). The model was a quasi-Poisson GAMM with log link function. Based on QIC, support for including the noise level as a predictor of productivity was: weak; support for including the standard deviation of noise was: strong. 


\begin{tabular}{lrrr}
\hline name & QIC & delta QIC & Akaike weight \\
\hline Full Model & 1031.74 & 0.00 & 0.34 \\
No Mean Noise Term & 1032.01 & 0.27 & 0.29 \\
No Noise Terms & 1032.83 & 1.09 & 0.19 \\
No Noise Variability Term & 1033.03 & 1.29 & 0.18 \\
\hline
\end{tabular}

Table A4.119: Model selection information for the productivity model for the Rufous Hummingbird (Selasphorus rufus). The model was a quasi-Poisson GAMM with log link function. Based on QIC, support for including the noise level as a predictor of productivity was: weak; support for including the standard deviation of noise was: weak.

\begin{tabular}{lrrr}
\hline name & QIC & delta QIC & Akaike weight \\
\hline No Noise Terms & 819.92 & 0.00 & 0.32 \\
No Mean Noise Term & 820.39 & 0.47 & 0.25 \\
No Noise Variability Term & 820.51 & 0.59 & 0.24 \\
Full Model & 820.98 & 1.06 & 0.19 \\
\hline
\end{tabular}

Table A4.120: Model selection information for the productivity model for the Scarlet Tanager (Piranga olivacea). The model was a quasi-Poisson GAMM with log link function. Based on QIC, support for including the noise level as a predictor of productivity was: none; support for including the standard deviation of noise was: none.

\begin{tabular}{lrrr}
\hline name & QIC & delta QIC & Akaike weight \\
\hline No Noise Terms & 4868.38 & 0.00 & 0.36 \\
No Noise Variability Term & 4869.11 & 0.73 & 0.25 \\
No Mean Noise Term & 4869.28 & 0.90 & 0.23 \\
Full Model & 4870.11 & 1.73 & 0.15 \\
\hline
\end{tabular}

Table A4.121: Model selection information for the productivity model for the Song Sparrow (Melospiza melodia). The model was a quasi-Poisson GAMM with log link function. Based on QIC, support for including the noise level as a predictor of productivity was: none; support for including the standard deviation of noise was: none. 


\begin{tabular}{lrrr}
\hline name & QIC & delta QIC & Akaike weight \\
\hline No Noise Terms & 2116.10 & 0.00 & 0.31 \\
No Mean Noise Term & 2116.44 & 0.34 & 0.26 \\
No Noise Variability Term & 2116.68 & 0.58 & 0.23 \\
Full Model & 2117.01 & 0.91 & 0.20 \\
\hline
\end{tabular}

Table A4.122: Model selection information for the productivity model for the Spotted Towhee (Pipilo maculatus). The model was a quasi-Poisson GAMM with log link function. Based on QIC, support for including the noise level as a predictor of productivity was: none; support for including the standard deviation of noise was: none.

\begin{tabular}{lrrr}
\hline name & QIC & delta QIC & Akaike weight \\
\hline No Noise Variability Term & 3042.85 & 0.00 & 0.96 \\
No Noise Terms & 3049.92 & 7.06 & 0.03 \\
Full Model & 3051.22 & 8.37 & 0.01 \\
No Mean Noise Term & 3057.06 & 14.21 & 0.00 \\
\hline
\end{tabular}

Table A4.123: Model selection information for the productivity model for the Swainson's Thrush (Catharus ustulatus). The model was a quasi-Poisson GAMM with log link function. Based on QIC, support for including the noise level as a predictor of productivity was: strong; support for including the standard deviation of noise was: none.

\begin{tabular}{lrrr}
\hline name & QIC & delta QIC & Akaike weight \\
\hline Full Model & 153.32 & 0.00 & 0.48 \\
No Mean Noise Term & 154.09 & 0.77 & 0.33 \\
No Noise Variability Term & 155.76 & 2.44 & 0.14 \\
No Noise Terms & 158.14 & 4.82 & 0.04 \\
\hline
\end{tabular}

Table A4.124: Model selection information for the productivity model for the Townsend's Solitaire (Myadestes townsendi). The model was a quasi-Poisson GAMM with log link function. Based on QIC, support for including the noise level as a predictor of productivity was: weak; support for including the standard deviation of noise was: strong.

\begin{tabular}{lrrr}
\hline name & QIC & delta QIC & Akaike weight \\
\hline Full Model & 391.61 & 0.00 & 0.29 \\
No Mean Noise Term & 391.80 & 0.18 & 0.27 \\
No Noise Terms & 391.95 & 0.33 & 0.25 \\
No Noise Variability Term & 392.38 & 0.77 & 0.20 \\
\hline
\end{tabular}

Table A4.125: Model selection information for the productivity model for the Townsend's Warbler (Setophaga townsendi). The model was a quasi-Poisson GAMM with log link function. Based on QIC, support for including the noise level as a predictor of productivity was: weak; support for including the standard deviation of noise was: weak. 


\begin{tabular}{lrrr}
\hline name & QIC & delta QIC & Akaike weight \\
\hline No Noise Terms & 2349.02 & 0.00 & 0.45 \\
No Mean Noise Term & 2349.50 & 0.48 & 0.36 \\
Full Model & 2351.77 & 2.75 & 0.11 \\
No Noise Variability Term & 2352.52 & 3.49 & 0.08 \\
\hline
\end{tabular}

Table A4.126: Model selection information for the productivity model for the Warbling Vireo (Vireo gilvus). The model was a quasi-Poisson GAMM with log link function. Based on QIC, support for including the noise level as a predictor of productivity was: none; support for including the standard deviation of noise was: none.

\begin{tabular}{lrrr}
\hline name & QIC & delta QIC & Akaike weight \\
\hline No Noise Variability Term & 1166.87 & 0.00 & 0.44 \\
Full Model & 1167.23 & 0.36 & 0.37 \\
No Noise Terms & 1169.83 & 2.96 & 0.10 \\
No Mean Noise Term & 1170.16 & 3.29 & 0.09 \\
\hline
\end{tabular}

Table A4.127: Model selection information for the productivity model for the White-breasted Nuthatch (Sitta carolinensis). The model was a quasi-Poisson GAMM with log link function. Based on QIC, support for including the noise level as a predictor of productivity was: strong; support for including the standard deviation of noise was: none.

\begin{tabular}{lrrr}
\hline name & QIC & delta QIC & Akaike weight \\
\hline No Noise Variability Term & 257.58 & 0.00 & 0.37 \\
Full Model & 258.04 & 0.46 & 0.29 \\
No Noise Terms & 258.71 & 1.13 & 0.21 \\
No Mean Noise Term & 259.58 & 2.00 & 0.13 \\
\hline
\end{tabular}

Table A4.128: Model selection information for the productivity model for the Western Bluebird (Sialia mexicana). The model was a quasi-Poisson GAMM with log link function. Based on QIC, support for including the noise level as a predictor of productivity was: weak; support for including the standard deviation of noise was: none.

\begin{tabular}{lrrr}
\hline name & QIC & delta QIC & Akaike weight \\
\hline No Mean Noise Term & 499.78 & 0.00 & 0.58 \\
Full Model & 500.59 & 0.81 & 0.39 \\
No Noise Terms & 506.22 & 6.43 & 0.02 \\
No Noise Variability Term & 507.64 & 7.85 & 0.01 \\
\hline
\end{tabular}

Table A4.129: Model selection information for the productivity model for the Western ScrubJay (Aphelocoma californica). The model was a quasi-Poisson GAMM with log link function. Based on QIC, support for including the noise level as a predictor of productivity was: none; support for including the standard deviation of noise was: strong. 


\begin{tabular}{lrrr}
\hline name & QIC & delta QIC & Akaike weight \\
\hline No Noise Variability Term & 1909.26 & 0.00 & 0.31 \\
Full Model & 1909.53 & 0.27 & 0.27 \\
No Mean Noise Term & 1909.86 & 0.60 & 0.23 \\
No Noise Terms & 1910.13 & 0.87 & 0.20 \\
\hline
\end{tabular}

Table A4.130: Model selection information for the productivity model for the Western Tanager (Piranga ludoviciana). The model was a quasi-Poisson GAMM with log link function. Based on QIC, support for including the noise level as a predictor of productivity was: weak; support for including the standard deviation of noise was: none.

\begin{tabular}{lrrr}
\hline name & QIC & delta QIC & Akaike weight \\
\hline Full Model & 2398.76 & 0.00 & 0.97 \\
No Noise Variability Term & 2407.17 & 8.41 & 0.01 \\
No Mean Noise Term & 2407.82 & 9.06 & 0.01 \\
No Noise Terms & 2414.32 & 15.57 & 0.00 \\
\hline
\end{tabular}

Table A4.131: Model selection information for the productivity model for the White-eyed Vireo (Vireo griseus). The model was a quasi-Poisson GAMM with log link function. Based on QIC, support for including the noise level as a predictor of productivity was: strong; support for including the standard deviation of noise was: strong.

\begin{tabular}{lrrr}
\hline name & QIC & delta QIC & Akaike weight \\
\hline No Mean Noise Term & 2165.74 & 0.00 & 0.26 \\
Full Model & 2165.81 & 0.07 & 0.25 \\
No Noise Variability Term & 2165.83 & 0.09 & 0.25 \\
No Noise Terms & 2165.96 & 0.22 & 0.23 \\
\hline
\end{tabular}

Table A4.132: Model selection information for the productivity model for the Wilson's Warbler (Cardellina pusilla). The model was a quasi-Poisson GAMM with log link function. Based on QIC, support for including the noise level as a predictor of productivity was: none; support for including the standard deviation of noise was: weak.

\begin{tabular}{lrrr}
\hline name & QIC & delta QIC & Akaike weight \\
\hline No Noise Variability Term & 3185.32 & 0.00 & 0.50 \\
Full Model & 3186.78 & 1.46 & 0.24 \\
No Noise Terms & 3187.38 & 2.06 & 0.18 \\
No Mean Noise Term & 3188.82 & 3.51 & 0.09 \\
\hline
\end{tabular}

Table A4.133: Model selection information for the productivity model for the Wood Thrush (Hylocichla mustelina). The model was a quasi-Poisson GAMM with log link function. Based on QIC, support for including the noise level as a predictor of productivity was: strong; support for including the standard deviation of noise was: none. 


\begin{tabular}{lrrr}
\hline name & QIC & delta QIC & Akaike weight \\
\hline No Mean Noise Term & 815.31 & 0.00 & 0.29 \\
No Noise Terms & 815.49 & 0.19 & 0.26 \\
Full Model & 815.73 & 0.42 & 0.23 \\
No Noise Variability Term & 815.83 & 0.52 & 0.22 \\
\hline
\end{tabular}

Table A4.134: Model selection information for the productivity model for the Wrentit (Chamaea fasciata). The model was a quasi-Poisson GAMM with log link function. Based on QIC, support for including the noise level as a predictor of productivity was: none; support for including the standard deviation of noise was: weak.

\begin{tabular}{lrrr}
\hline name & QIC & delta QIC & Akaike weight \\
\hline No Mean Noise Term & 279.48 & 0.00 & 0.42 \\
No Noise Terms & 280.79 & 1.30 & 0.22 \\
Full Model & 280.98 & 1.49 & 0.20 \\
No Noise Variability Term & 281.54 & 2.05 & 0.15 \\
\hline
\end{tabular}

Table A4.135: Model selection information for the productivity model for the White-throated Sparrow (Zonotrichia albicollis). The model was a quasi-Poisson GAMM with log link function. Based on QIC, support for including the noise level as a predictor of productivity was: none; support for including the standard deviation of noise was: weak.

\begin{tabular}{lrrr}
\hline name & QIC & delta QIC & Akaike weight \\
\hline No Noise Terms & 2300.70 & 0.00 & 0.32 \\
No Noise Variability Term & 2301.09 & 0.39 & 0.26 \\
No Mean Noise Term & 2301.33 & 0.63 & 0.23 \\
Full Model & 2301.66 & 0.96 & 0.19 \\
\hline
\end{tabular}

Table A4.136: Model selection information for the productivity model for the Yellow-breasted Chat (Icteria virens). The model was a quasi-Poisson GAMM with log link function. Based on QIC, support for including the noise level as a predictor of productivity was: none; support for including the standard deviation of noise was: none.

\begin{tabular}{lrrr}
\hline name & QIC & delta QIC & Akaike weight \\
\hline No Noise Variability Term & 335.49 & 0.00 & 0.47 \\
Full Model & 336.51 & 1.02 & 0.28 \\
No Noise Terms & 337.76 & 2.28 & 0.15 \\
No Mean Noise Term & 338.80 & 3.31 & 0.09 \\
\hline
\end{tabular}

Table A4.137: Model selection information for the productivity model for the Yellow-billed Cuckoo (Coccyzus americanus). The model was a quasi-Poisson GAMM with log link function. Based on QIC, support for including the noise level as a predictor of productivity was: strong; support for including the standard deviation of noise was: none. 


\begin{tabular}{lrrr}
\hline name & QIC & delta QIC & Akaike weight \\
\hline No Noise Terms & 3126.09 & 0.00 & 0.31 \\
No Mean Noise Term & 3126.61 & 0.52 & 0.24 \\
Full Model & 3126.75 & 0.65 & 0.22 \\
No Noise Variability Term & 3126.76 & 0.67 & 0.22 \\
\hline
\end{tabular}

Table A4.138: Model selection information for the productivity model for the Yellow Warbler (Setophaga petechia). The model was a quasi-Poisson GAMM with log link function. Based on QIC, support for including the noise level as a predictor of productivity was: none; support for including the standard deviation of noise was: none. 


\section{Appendix 5 \\ Tables for measurement error models}

\section{Description}

The tables below present the full models for:

1. Productivity coefficients as a function of abundance coefficients (weighted mean noise level)

2. Productivity coefficients as a function of abundance coefficients (noise standard deviation)

3. Productivity coefficients as a function of song acoustic characteristics and life-history traits (weighted mean noise level)

4. Productivity coefficients as a function of song acoustic characteristics and life-history traits (noise standard deviation)

In all cases, all model selection criteria examined indicated that the best model was the intercept-only model (with no predictors). In each table, "CI (low)" and "CI (high)" indicate the lower and upper bounds of 95\% credible intervals around the estimates. (Note: in most cases the shrinkage prior has resulted in slope parameter estimates that are approximately zero.) 
Table 1: Model coefficients for measurement error model to predict productivity regression coefficients as a function of abundance regression coefficients, for effects of weighted mean noise level.

\begin{tabular}{l|r|r|r|r}
\hline Term & Estimate & Standard Error & CI (low) & CI (high) \\
\hline Intercept & 0.0099 & 0.0039 & 0.0022 & 0.0175 \\
\hline Abundance & 0.0000 & 0.0000 & 0.0000 & 0.0000 \\
\hline
\end{tabular}

Table 2: Model coefficients for measurement error model to predict productivity regression coefficients as a function of abundance regression coefficients, for effects of noise standard deviation.

\begin{tabular}{l|r|r|r|r}
\hline Term & Estimate & Standard Error & CI (low) & CI (high) \\
\hline Intercept & 0.0089 & 0.021 & -0.0324 & 0.0498 \\
\hline Abundance & 0.0000 & 0.000 & 0.0000 & 0.0000 \\
\hline
\end{tabular}

Table 3: Model coefficients for measurement error model to predict productivity regression coefficients as a function of song acoustic characteristics and life-history traits, for effects of weighted mean noise level.

\begin{tabular}{l|r|r|r|r}
\hline Term & Estimate & Standard Error & CI (low) & CI (high) \\
\hline Intercept & 0.0109 & 0.0038 & 0.0034 & 0.0184 \\
\hline Freq5 & 0.0000 & 0.0000 & 0.0000 & 0.0000 \\
\hline Freq95 & 0.0000 & 0.0000 & 0.0000 & 0.0000 \\
\hline IQR.BW & 0.0000 & 0.0000 & 0.0000 & 0.0000 \\
\hline Peak & 0.0000 & 0.0000 & 0.0000 & 0.0000 \\
\hline Q3 & 0.0000 & 0.0000 & 0.0000 & 0.0000 \\
\hline HabitatGrassland & 0.0000 & 0.0000 & 0.0000 & 0.0000 \\
\hline HabitatOpenWoodland & 0.0000 & 0.0000 & 0.0000 & 0.0000 \\
\hline HabitatScrub & 0.0000 & 0.0000 & 0.0000 & 0.0000 \\
\hline HabitatTown & 0.0000 & 0.0000 & 0.0000 & 0.0000 \\
\hline Insectsyes & 0.0000 & 0.0000 & 0.0000 & 0.0000 \\
\hline NestingGround & 0.0000 & 0.0000 & 0.0000 & 0.0000 \\
\hline NestingShrub & 0.0000 & 0.0000 & 0.0000 & 0.0000 \\
\hline NestingTree & 0.0000 & 0.0000 & 0.0000 & 0.0000 \\
\hline MigrationNonMMigrator & 0.0000 & 0.0000 & 0.0000 & 0.0000 \\
\hline MigrationPartialMigrator & 0.0000 & 0.0000 & 0.0000 & 0.0000 \\
\hline
\end{tabular}


Table 4: Model coefficients for measurement error model to predict productivity regression coefficients as a function of song acoustic characteristics and life-history traits, for effects of noise standard deviation.

\begin{tabular}{l|r|r|r|r}
\hline Term & Estimate & Standard Error & CI (low) & CI (high) \\
\hline Intercept & 0.0073 & 0.0213 & -0.0344 & 0.0488 \\
\hline Freq5 & 0.0000 & 0.0000 & 0.0000 & 0.0000 \\
\hline Freq95 & 0.0000 & 0.0000 & 0.0000 & 0.0000 \\
\hline IQR.BW & 0.0000 & 0.0000 & 0.0000 & 0.0000 \\
\hline Peak & 0.0000 & 0.0000 & 0.0000 & 0.0000 \\
\hline Q3 & 0.0000 & 0.0000 & 0.0000 & 0.0000 \\
\hline HabitatGrassland & 0.0000 & 0.0000 & 0.0000 & 0.0000 \\
\hline HabitatOpenWoodland & 0.0000 & 0.0000 & 0.0000 & 0.0000 \\
\hline HabitatScrub & 0.0000 & 0.0000 & 0.0000 & 0.0000 \\
\hline HabitatTown & 0.0000 & 0.0000 & 0.0000 & 0.0000 \\
\hline Insectsyes & 0.0000 & 0.0000 & 0.0000 & 0.0000 \\
\hline NestingGround & 0.0000 & 0.0000 & 0.0000 & 0.0000 \\
\hline NestingShrub & 0.0000 & 0.0000 & 0.0000 & 0.0000 \\
\hline NestingTree & 0.0000 & 0.0000 & 0.0000 & 0.0000 \\
\hline MigrationNonMMigrator & 0.0000 & 0.0000 & 0.0000 & 0.0000 \\
\hline MigrationPartialMigrator & 0.0000 & 0.0000 & 0.0000 & 0.0000 \\
\hline
\end{tabular}

Table 5: Model coefficients for measurement error model to predict abundance regression coefficients as a function of song acoustic characteristics and life-history traits, for effects of weighted mean noise level.

\begin{tabular}{l|r|r|r|r}
\hline Term & Estimate & Standard Error & CI (low) & CI (high) \\
\hline Intercept & 0.0073 & 0.0213 & -0.0344 & 0.0488 \\
\hline Freq5 & 0.0000 & 0.0000 & 0.0000 & 0.0000 \\
\hline Freq95 & 0.0000 & 0.0000 & 0.0000 & 0.0000 \\
\hline IQR.BW & 0.0000 & 0.0000 & 0.0000 & 0.0000 \\
\hline Peak & 0.0000 & 0.0000 & 0.0000 & 0.0000 \\
\hline Q3 & 0.0000 & 0.0000 & 0.0000 & 0.0000 \\
\hline HabitatGrassland & 0.0000 & 0.0000 & 0.0000 & 0.0000 \\
\hline HabitatOpenWoodland & 0.0000 & 0.0000 & 0.0000 & 0.0000 \\
\hline HabitatScrub & 0.0000 & 0.0000 & 0.0000 & 0.0000 \\
\hline HabitatTown & 0.0000 & 0.0000 & 0.0000 & 0.0000 \\
\hline Insectsyes & 0.0000 & 0.0000 & 0.0000 & 0.0000 \\
\hline NestingGround & 0.0000 & 0.0000 & 0.0000 & 0.0000 \\
\hline NestingShrub & 0.0000 & 0.0000 & 0.0000 & 0.0000 \\
\hline NestingTree & 0.0000 & 0.0000 & 0.0000 & 0.0000 \\
\hline MigrationNonMMigrator & 0.0000 & 0.0000 & 0.0000 & 0.0000 \\
\hline MigrationPartialMigrator & 0.0000 & 0.0000 & 0.0000 & 0.0000 \\
\hline
\end{tabular}


Table 6: Model coefficients for measurement error model to predict abundance regression coefficients as a function of song acoustic characteristics and life-history traits, for effects of noise standard deviation.

\begin{tabular}{l|r|r|r|r}
\hline Term & Estimate & Standard Error & CI (low) & CI (high) \\
\hline Intercept & 0.0866 & 0.0251 & 0.0377 & 0.1364 \\
\hline Freq5 & 0.0000 & 0.0000 & 0.0000 & 0.0000 \\
\hline Freq95 & 0.0000 & 0.0000 & 0.0000 & 0.0000 \\
\hline IQR.BW & 0.0000 & 0.0000 & 0.0000 & 0.0000 \\
\hline Peak & 0.0000 & 0.0000 & 0.0000 & 0.0000 \\
\hline Q3 & 0.0000 & 0.0000 & 0.0000 & 0.0000 \\
\hline HabitatGrassland & 0.0000 & 0.0000 & 0.0000 & 0.0000 \\
\hline HabitatOpenWoodland & 0.0000 & 0.0000 & 0.0000 & 0.0000 \\
\hline HabitatScrub & 0.0000 & 0.0000 & 0.0000 & 0.0000 \\
\hline HabitatTown & 0.0000 & 0.0000 & 0.0000 & 0.0000 \\
\hline Insectsyes & 0.0000 & 0.0000 & 0.0000 & 0.0000 \\
\hline NestingGround & 0.0000 & 0.0000 & 0.0000 & 0.0000 \\
\hline NestingShrub & 0.0000 & 0.0000 & 0.0000 & 0.0000 \\
\hline NestingTree & 0.0000 & 0.0000 & 0.0000 & 0.0000 \\
\hline MigrationNonMMigrator & 0.0000 & 0.0000 & 0.0000 & 0.0000 \\
\hline MigrationPartialMigrator & 0.0000 & 0.0000 & 0.0000 & 0.0000 \\
\hline
\end{tabular}


Appendix 6. R code to develop and analyze noise models for a single species

Please click here to download file 'appendix6.rmd'. 
Avian Conservation and Ecology 15(2): 4

http://www.ace-eco.org/vol15/iss2/art4/

Appendix 7. R code for the measurement error models.

Please click here to download file 'appendix7.rnw'. 